

\title{
Fundamental Properties of Bond Prices in Models of the Short-Term Rate
}

\author{
Antonio Mele \\ Queen Mary, University of London
}

June 2002

\begin{abstract}
This paper develops restrictions that arbitrage-constrained bond prices impose on the short-term rate process in order to be consistent with given dynamic properties of the term-structure of interest rates. The central focus is the relationship between bond prices and the short-term rate volatility. In both scalar and multidimensional diffusion settings, typical relationships between bond prices and volatility are generated by joint restrictions on the risk-neutralized drift functions of the state variables and convexity of bond prices with respect to the short-term rate. The theory is illustrated by several examples and is partially extended to accommodate the occurrence of jumps and default.
\end{abstract}

JEL Codes: C61; G12; G13

I dedicate this work to the memory of my beloved father Ettore, who passed away when I started to produce its latest revisions. The first draft of the paper was written when I was a Visiting Fellow at the Economics Department of Princeton University (Spring 2000). I thank Yacine Aït-Sahalia and José Scheinkman for stimulating conversations and helpful suggestions. I am also grateful to Kyriakos Chourdakis, Alan Lewis and Jaouad Sahbani for valuable remarks made on early versions of the manuscript, and seminar participants at the University of Exeter and at the 2002 American Finance Association meeting, especially Francis Longstaff, for useful comments. I benefited considerably from the detailed comments of an anonymous referee, the Editor Kenneth Singleton, and the Executive Editor Maureen O'Hara, particularly from their suggestions on how to improve the presentation and how to consolidate the analysis of multifactor models and jumps phenomena. This work was partly supported by a Fulbright research fellowship that I gratefully acknowledge. The usual disclaimer applies. 
A standard approach to modeling the term-structure of interest rates consists in deriving sets of arbitrage-free bond prices using as an input an exogenously given short-term rate process. While this approach is perhaps the best-known since the influential articles by Merton (1973) and Vasicek (1977), there is not yet theoretically sound work trying to answer in a general way to such fundamental questions as: When are bond prices a decreasing function of the short-term rate? When are bond prices a strictly convex function of the short-term rate? Are bond prices a decreasing function of the shortterm rate volatility? This paper demonstrates that it is possible to develop results that partially answer these questions in relation to all sets of economically admissible, i.e. no-arbitrage, bond prices.

The central purposes of this paper share common features with objectives and methods developed in the option pricing literature since the seminal work of Merton (1973), Cox and Ross (1976) and Jagannathan (1984): the objectives there are to uncover general properties of contingent claim prices when, in addition to the assumption of absence of arbitrage opportunities, a few more assumptions are made as regards the dynamics of the price of the asset underlying the various contractual provisions. Related papers that also derive general properties of bond prices while imposing minimal assumptions on the state processes are Dybvig, Ingersoll and Ross (1996) and Dunn and Spatt (1999), but the results of the present article in the term-structure domain are totally new.

The class of stochastic processes typically used to analyze general properties of option prices is the one of diffusion processes, as in the relatively more recent contributions of Bergman, Grundy and Wiener (1996), Bajeux-Besnainou and Rochet (1996, section 5), Romano and Touzi (1997) and El Karoui, Jeanblanc-Picqué and Shreve (1998). As is well-known, diffusion processes are also the workhorse in the term-structure field. Yet, the main concern of researchers was to develop a variety of models aiming at increasing precision in explaining empirically the short-term rate dynamics (e.g., Chan et al. (1992), Aït-Sahalia (1996a), Conley et al. (1997), Andersen and Lund (1997a), Gallant and Tauchen (1998)) as well as the implications that these specific models have on the yield curve (e.g., Aït-Sahalia (1996b), Stanton (1997), Andersen and Lund (1997b), Boudoukh et al. (1998)). Since it is difficult to ascertain empirically what the data generating process is, however, it is natural to group and classify bond prices properties corresponding to general qualitative features of the primitive state processes dynamics.

In the present paper, the only assumption made is that the primitive state processes are diffusion processes satisfying some basic regularity conditions. Precisely, in the framework analyzed here, the short-term rate and its instantaneous, stochastic volatility form a joint Markov process that is a sufficient statistic for the state variables of the economy. ${ }^{1}$ As the title suggests, the main concern is to develop "fundamental" results relating equilibrium bond prices movements to changes of the short-term rate and its instantaneous volatility. This paper does not cover, for instance, issues concerning the origins of a given shape of the yield curve (e.g., uniformly rising, uniformly decreasing, humped,

\footnotetext{
${ }^{1}$ Surveys on continuous time stochastic volatility option pricing models can be found in Ghysels, Harvey and Renault (1996), Lewis (2000) or Fouque, Papanicolaou and Sircar (2000). Recent work on specification, estimation and filtering methods applied to stochastic volatility models for the short-term rate include Gallant and Tauchen (1998), Dai and Singleton (2000) and Mele and Fornari (2000).
} 
inverted-humped, ...). Furthermore, as noted above, this paper only examines the case in which the short-term rate and its instantaneous volatility are a sufficient statistic for the state variables of the economy. As a result of this, this paper does not consider, for example, the continuous time non-Markovian approach as pioneered by Heath, Jarrow and Morton (1992); however, extending the approach followed here to more general situations is a promising area for future research. A step in that direction is made in section 5, where it is shown how easily this paper's approach may be extended to treat multifactor models with unobserved factors that do not necessarily have an immediate economic interpretation (e.g., models with "stochastic central tendency").

The starting point of the paper is the situation in which the short-term rate follows a strictly stationary scalar diffusion. This is the simplest analytical situation in which bond prices are expected to display quite intuitive properties. One of these properties is that bond prices are decreasing in the short-term rate, and another one is that bond prices are convex in the short-term rate. Virtually every textbook does illustrate the importance of these two properties (see, e.g., Campbell, Lo and MacKinlay (1997, p. 406)); when the former property holds, for instance, convexity may be desirable to an investor because it makes a position more valuable after a price change than its duration value suggests. Other related and yet relatively unexplored issues cover the relationships between bond prices, volatility and patterns of risk aversion.

In the scalar diffusion setting, I prove the following properties:

I) Bond prices are always decreasing in the short-term rate (see section 3.1, proposition 1);

II) Bond prices are convex in the short-term rate under a bound on the degree of convexity of the short-term rate risk-neutralized drift function (see section 3.1, propositions 1 and 2);

III) The level of the term-structure of interest rates always increases with risk aversion (see section 3.2, proposition 3);

IV) At least at short maturity dates, bond prices decrease with volatility whenever the market risk-premium has a strictly positive value and increases with volatility (see section 3.3).

Property I) is relatively simple to show heuristically. It is due to a property of scalar diffusions, the so-called "no-crossing" property, which states that all paths of a scalar diffusion are non-decreasing in the initial state. Bergman, Grundy and Wiener (1996) were the first to note that in a scalar diffusion setting, the no-crossing property implies that option prices are increasing in the underlying stock price. However, they also showed that such a feature of option prices needs not to hold in more complicated multidimensional settings such as the stochastic volatility setting. Given these simple but deep insights, one may expect that similar phenomena should also take place in the term-structure domain analyzed in this article. Here I prove that properties I) and II) have natural counterparts in certain cases of such more complicated settings: 
V) In multifactor models with stochastic volatility, properties I) and II) still hold if the unobservable factor risk-neutral dynamics does not depend on the short-term rate (see section 4.1, proposition 4);

VI) In multifactor models with stochastic volatility, bond prices are always decreasing in the short-term rate at short maturity dates (see section 4.1, proposition 5);

While I identify important cases in which property VI) holds independently of the current level of the state variables (see appendix C), I also provide conditions and examples under which bond prices are increasing in the short-term rate at medium-long maturity dates. Such conditions and examples are presented in section 4 (see section 4.1, table 1). Section 4 also contains the central results of this paper on how rationally formed bond prices react to (stochastic) volatility changes. ${ }^{2}$ I prove two general properties:

VII) If the drift function of the short-term rate process under the risk-neutral measure is decreasing in volatility, and bond prices are decreasing and convex in the shortterm rate, then positive volatility changes make bond prices increase (see section 4.2 , condition (11));

VIII) Positive volatility changes make bond prices decrease (increase) at short maturity dates if the drift function of the short-term rate process under the risk-neutral measure is increasing (decreasing) in volatility (see section 4.2, proposition 6 ).

Properties VII) and VIII) are in tight connection with property IV) stated for the scalar diffusion case. Property IV), for instance, is rather different from properties known in the option pricing field, where derivative prices are increasing in the volatility of the underlying asset price (as in the celebrated Black and Scholes (1973) model). Similarly, in the stochastic volatility option pricing domain, convexity of option prices with respect to the underlying asset price is known to be a necessary condition for option prices to be increasing in volatility (see Romano and Touzi (1997)). Section 2 then provides a heuristic explanation of properties VII) and VIII), and sections 3.3 and 4.2 and appendices B,C and $\mathrm{D}$ contain conditions and examples for bond prices and volatility to be negatively related at any arbitrary maturity date, thus strengthening properties IV) and VIII). As regards the scalar diffusion case, for example, in section 3.3 I show that bond prices are always decreasing in volatility with a sufficiently high level of the market risk-premium. Similar conditions and examples are developed in section 4.2 in the setting of stochastic volatility models.

Section 5 shows that the previous properties of diffusion models with random volatility also hold in more complicated three factor diffusion settings. As an example, property VIII) is shown to have a natural counterpart in such settings (see proposition 7). The only exception is property VII), which is replaced by a property that imposes a more severe condition on the joint factor dynamics (see section 5, proposition 8).

\footnotetext{
${ }^{2}$ For previous numerical exercises aiming at unveiling the relationship between bond prices and volatility, see Litterman, Scheinkman and Weiss (1991), Chen (1996), Andersen and Lund (1997b), and Mele and Fornari (2000, chapter 5).
} 
A number of examples are provided that illustrate all these properties, and that shed new light on very familiar models. Perhaps the most important examples are the Longstaff and Schwartz (1992) model as well as two three-factor models more recently formulated by Dai and Singleton (2000). These models are affine and their solution is therefore very easy to implement. As Lewis (2000, p. 4) suggested in relation to models in the option pricing area, models of this kind are economically very important: not only are these models easy to solve, but their solution is also "typical", i.e. it displays the main qualitative properties that we expect in more general (nonlinear) settings.

Section 6 studies the robustness of the theory to the introduction of jumps phenomena and the possibility of default. Results are essentially unaffected as long as the parameters of the jump size distribution and various hazard rates are "sufficiently independent" of the level of the state variables (see proposition 9). Section 6 also provides comparative statics on the hazard rate governing the jumps generating process.

In addition to providing a series of testable implications of any diffusion model of the short-term rate in the setting of stochastic volatility, the previously listed properties can also be used to implement comparative statics results. Such results constitute indeed the final contribution of the paper, and are collected in section 7 . The objective will be to analyze how bond price functions change in the thought-experiment in which we change risk-premia, the "volatility of volatility", or the correlation between volatility and the short-term rate. In all cases, it will be shown that the relationship between bond prices and volatility is crucial in determining such comparative statics results.

To summarize, the article is organized as follows: the next section introduces the model's primitives and section 2 provides a selected heuristic overview of the paper's results; section 3 contains preliminary results pertaining to scalar diffusions; section 4 presents results concerning stochastic volatility models; section 5 makes an extension, and considers a three-factor model in which the drift function of the short-term rate may exhibit a stochastic central tendency; section 6 deals with cases in which the state variables exhibit discontinuities; section 7 provides comparative statics results relating bond prices to risk-premia, the volatility of volatility and correlation issues; section 8 concludes; seven appendices gather proofs and examples omitted in the main text.

\section{The model}

In the basic model considered here, the short-term rate and its instantaneous volatility form together a sufficient statistic for the state variables generating uncertainty in the economy. Such a model is very important to study in the first place because it displays in a transparent way many interesting qualitative properties of more complex three-factor models. The reader may refer to section 5 to learn additional properties that are specific to three-factor models.

I begin with describing the risk-neutral measure space under which the discounted bond prices are $Q$-martingales. This is $(\Omega, \mathcal{F}, \mathbb{F}, Q)$, where $\mathbb{F}=\{\mathcal{F}(\tau)\}_{\tau \in[t, T]}$ is taken to be the $Q$-augmentation of the natural filtration $\sigma((W, B)(u), u \leq \tau)$ generated by two independent $Q$-Brownian motions $W, B$ (with $\mathcal{F}=\mathcal{F}(T)$ and $T<\infty$ ). I suppose that the short-term rate $r$ and volatility $y$ are diffusion processes, i.e. Markov processes with continuous sample paths (see, e.g., Karlin and Taylor (1981, p. 157)). They form a 
strong solution of the following stochastic differential system:

$$
\left(\begin{array}{l}
d r(\tau) \\
d y(\tau)
\end{array}\right)=\left(\begin{array}{l}
b(r(\tau), y(\tau)) \\
\varphi(r(\tau), y(\tau))
\end{array}\right) d \tau+\left(\begin{array}{ll}
\sigma^{(1)}(r(\tau), y(\tau)) & \sigma^{(2)}(r(\tau), y(\tau)) \\
\psi^{(1)}(r(\tau), y(\tau)) & \psi^{(2)}(r(\tau), y(\tau))
\end{array}\right)\left(\begin{array}{c}
d W(\tau) \\
d B(\tau)
\end{array}\right)
$$

for $\tau \in(t, T]$, where $(r, y)$ take values in $\mathbb{R}_{++}$and $\mathbb{R},(r(t), y(t)) \equiv(x, s)$, and $b, \sigma^{(j)}, \varphi$ and $\psi^{(j)}$ are progressively $\mathcal{F}(\tau)$-measurable functions satisfying regularity conditions which ensure a strong solution (e.g., definition 2.1 p. 285 in Karatzas and Shreve (1991)) to the preceding system. Let $\sigma(r, y) \equiv \sum_{j=1}^{2} \sigma^{(j)}(r, y)^{2} / 2$ and $\psi(r, y) \equiv \sum_{j=1}^{2} \psi^{(j)}(r, y)^{2} / 2$. I suppose that $\partial \sigma / \partial y \geq 0$ and $\partial \sigma / \partial r \geq 0$.

As is well-known, many of the sufficient conditions that are given in standard textbooks to ensure strong-sense existence for (1) (e.g., Karatzas and Shreve (1991, theorem 2.9 p. 289)) fail to be satisfied in many interesting economic models. This is particularly true for models attempting to capture nonlinear features of the observed short-term rate dynamics (e.g., Chan et al. (1992), Aït-Sahalia (1996a,b), Conley et al. (1997), Stanton (1997)). Appendix A then provides a set of conditions that are less stringent than the usual ones, and that were originally used by Aït-Sahalia $(1996 a, b)$ to ensure that classical results for stochastic differential equations still hold for such nonlinear models. Not only do such conditions ensure strong uniqueness for (1), but they also enable one to apply the Feynman-Kac representation theorem to many of the objects I study below (see appendix A). One subset of these conditions is summarized in the following assumptions, that I shall be using throughout the paper.

Maintained assumptions. Functions $b, \varphi, \sigma, \psi$ are continuous and twice differentiable and the resulting derivatives are continuous.

The assumptions above are not sufficient to ensure strong uniqueness. In the scalar diffusion case, they can when they are coupled with a very mild technical assumption given in appendix A (see assumption A2) that ensures stationarity of the short-term rate. Only in the stochastic volatility cases will relatively more stringent conditions (e.g., global Lipschitz conditions) be imposed to the functions in (1) (see appendix A). ${ }^{3}$ In addition to be useful for the problem analyzed here, the conditions in appendix A also clarify the kind of assumptions that were implicitly made in the seminal work of Bergman, Grundy and Wiener (1996) in the option pricing domain. Finally, such conditions are of course only sufficient conditions. Stochastic volatility models that are known for not

\footnotetext{
${ }^{3}$ In the option pricing literature, both Romano and Touzi (1997) in the two-dimensional case (p. 401 and p. 406) and El Karoui, Jeanblanc-Picqué and Shreve (1998) in the scalar case (p. 101), for instance, require boundedness of the coefficients of the infinitesimal generator. Bergman, Grundy and Wiener (1996) assume (p. 1576) that claim prices can be computed via the Feynman-Kac representation theorem. However, the authors implicitly require much more than this, since they often make use of the Feynman-Kac representation theorem for the partial derivatives of the option price. See appendix A for a discussion of this issue within the framework that is treated here. In addition, as Lewis (2000) remarks (p. 71 footnote 1), even the Feynman-Kac representation of option prices fails when discounted claim prices are not martingales, as for instance in the case of volatility explosions that is treated in his chapter 9. The same caveat applies here.
} 
satisfying such conditions are not necessarily excluded by the analysis this paper presents. Alternatively, one can interpret the results of this paper as approximation results for this kind of models.

To relate the drift functions in (1) to the corresponding drift functions defined under the physical measure space, recall the well-known result stating that in the absence of arbitrage opportunities, there exist functions $\Lambda^{1}$ and $\Lambda^{2}$ such that the drift functions in (1) can be written as:

$$
\left\{\begin{array}{l}
b(r, y)=\widehat{b}(r, y)+\sum_{j=1}^{2} \sigma^{(j)}(r, y) \cdot \Lambda^{j}(r, y) \\
\varphi(r, y)=\widehat{\varphi}(r, y)-\sum_{j=1}^{2} \psi^{(j)}(r, y) \cdot \Lambda^{j}(r, y)
\end{array}\right.
$$

where $\widehat{b}(\cdot, \cdot)$ and $\widehat{\varphi}(\cdot, \cdot)$ denote the drift functions under the physical measure space, $\Lambda=$ $\left(\Lambda(\tau) \equiv\left(-\Lambda^{1}(r(\tau), y(\tau)), \Lambda^{2}(r(\tau), y(\tau))_{\tau \in[t, T]}\right.\right.$ is a $\mathcal{F}(\tau)$-adapted process that satisfies standard regularity conditions. ${ }^{4}$ Under a boundedness condition given in footnote 3 on $\Lambda$, and the condition that for all $(r, y) \in \mathbb{R}_{++} \times \mathbb{R}: \sigma^{(1)}(r, y)=\sigma^{(2)}(r, y)=\psi^{(1)}(r, y)=$ $\psi^{(2)}(r, y)=0, \sum_{j=1}^{2} \sigma^{(j)}(r, y) \Lambda^{(j)}(r, y)=\sum_{j=1}^{2} \psi^{(j)}(r, y) \Lambda^{(j)}(r, y)=0$, any otherwise arbitrary functional form of $\Lambda^{i}$ will prevent arbitrage opportunities similar to those first discussed by Cox, Ingersoll and Ross (CIR, henceforth) (1985, section 5).

Naturally, the fact that the technical starting point in (1) is the risk-neutral measure does not imply that only a risk-neutral world is being considered here. As is well-known, the economic interpretation of $\Lambda^{1}$ and $\Lambda^{2}$ is that of risk premia demanded by agents to be compensated for the stochastic fluctuations of the two Brownian motions in (1), and $\Lambda^{i}$ are both nil when agents are risk-neutral. Eqs. (2) thus summarize the "mapping" between the fundamentals (law of motion of the state variables and a model of riskaversion) and the risk-neutral drifts in (1). Consequently, all no-arbitrage restrictions in this article will be joint restrictions on both the law of motion of the state-variables under the physical measure and models of risk-aversion. ${ }^{5}$

Let $u(x, s, t, T)$ denote the rational price of a bond expiring at $T \geq t$ when the shortterm rate and its instantaneous volatility are $(x, s)$ at time $t$. The following partial

\footnotetext{
${ }^{4}$ Formally, the physical measure $P$ (say) and measure $Q$ are equivalent measures with RadonNikodym derivative of $Q$ with respect to $P$ on $\mathcal{F}(T)$ given by $d Q / d P=\exp \left(-\int_{t}^{T} \Lambda(r(\tau), y(\tau))\right.$ $\left.d U(\tau)-\int_{t}^{T}\|\Lambda(r(\tau), y(\tau))\|^{2} d \tau / 2\right)$, where $U=(W, B)^{\mathrm{T}}$ and the $\mathcal{F}(\tau)$-adapted $\{\Lambda(r(\tau), y(\tau))\}_{\tau \in[t, T]}$ process is taken to satisfy the Novikov's condition (see, e.g., Karatzas and Shreve (1991, corollary 5.13 p. 199)): $E\left\{\exp \left(\int_{t}^{T}\|\Lambda(r(\tau), y(\tau))\|^{2} d \tau / 2\right)\right\}<\infty$, where $E\{\cdot\}$ is the expectation operator taken under measure $P$.

${ }^{5}$ This observation is concretely illustrated several times in the article: see, for instance, eqs. (12), (17) and (18). Recent empirical studies focusing on the estimation of objects defined under the physical measure as well as the risk-neutral measure include Mele and Fornari (2000, chap. 5) and Dai and Singleton (2001) in the term-structure domain; and Chernov and Ghysles (2000) and Mele and Fornari (2001) in the option pricing field.
} 
differential equation will be satisfied by $u$ in the absence of arbitrage opportunities:

$$
\left\{\begin{array}{l}
0=\left(\frac{\partial}{\partial \tau}+L-r\right) u(r, y, \tau, T), \forall(r, y, \tau) \in \mathbb{R}_{++} \times \mathbb{R} \times[t, T) \\
u(r, y, T, T)=1, \forall(r, y) \in \mathbb{R}_{++} \times \mathbb{R}
\end{array}\right.
$$

where $\frac{\partial}{\partial \tau} \cdot+L$. is the usual infinitesimal generator of (1), with:

$$
L u=b u_{1}+\varphi u_{2}+\sigma u_{11}+\psi u_{22}+\left(\sigma^{(1)} \psi^{(1)}+\sigma^{(2)} \psi^{(2)}\right) u_{12},
$$

where $u_{1} \equiv \partial u / \partial r, u_{11} \equiv \partial^{2} u / \partial r^{2}, u_{2} \equiv \partial u / \partial y$, and so on. No transversality conditions are imposed here. ${ }^{6}$ Throughout this paper, it will also be assumed that the coefficients of the infinitesimal generator of (1) are such that the bond price and its partial derivatives can be computed via the celebrated Feynman-Kac representation theorem (e.g. Karatzas and Shreve (1991, p. 366)). Regularity conditions ensuring the feasibility of such a representation as well as related regularity conditions are spelled out in appendices $\mathrm{A}, \mathrm{B}$ and $\mathrm{C}$.

Let $\vartheta(\tau) \equiv \vartheta(\tau, z ; \omega), \tau \in[t, T]$ denote the solution flow of the first stochastic differential equation in (1) at $\tau$ starting at $z \equiv(x, s)$ at the point $\omega \in \Omega$. Under the regularity conditions mentioned before, there is a unique $C^{2,1}\left(\mathbb{R}_{++} \times \mathbb{R},[0, T]\right)$ solution of (3) that admits the Feynman-Kac stochastic representation:

$$
u(x, s, t, T)=\mathbb{E}\left\{\exp \left(-\int_{t}^{T} \vartheta(\tau) d \tau\right)\right\},
$$

where $\mathbb{E}$ is the expectation operator taken under measure $Q$.

By differentiating the bond price in (4),

$$
u_{1}(x, s, t, T)=-\mathbb{E}\left\{\left(\int_{t}^{T} \frac{\partial \vartheta}{\partial x}(\tau) d \tau\right) \cdot \exp \left(-\int_{t}^{T} \vartheta(\tau) d \tau\right)\right\},
$$

and

$$
\begin{aligned}
u_{11}(x, s, t, T)= & \mathbb{E}\left\{\left(\int_{t}^{T} \frac{\partial \vartheta}{\partial x}(\tau) d \tau\right)^{2} \cdot \exp \left(-\int_{t}^{T} \vartheta(\tau) d \tau\right)\right\} \\
& -\mathbb{E}\left\{\left(\int_{t}^{T} \frac{\partial^{2} \vartheta}{\partial x^{2}}(\tau) d \tau\right) \cdot \exp \left(-\int_{t}^{T} \vartheta(\tau) d \tau\right)\right\},
\end{aligned}
$$

where the "sensitivity processes" $\partial \vartheta / \partial x, \partial \vartheta / \partial s$, etc. are taken to share the same "diffusion" properties as (1) and in particular are such that $\exists T_{+}>t: \mathbb{E}\left\{\sup _{T}\left(\frac{1}{T-t} \int_{t}^{T}(\partial \vartheta / \partial x)(\tau)\right.\right.$ $d \tau)\}<\infty$ for all $T<T_{+}$, and similarly for the other sensitivity processes. These last conditions are very mild, and will be used to study the local properties of (4). Additional technical conditions that do not have an immediate economic interpretation are that $\forall(x, s) \in \mathbb{R}_{++} \times \mathbb{R}, \exists T_{+}>t$ such that, for all $\tau \in\left[t, T_{+}\right]$,

\footnotetext{
${ }^{6}$ As Longstaff (1992) pointed out in a related context, if 0 and $\infty$ (resp. $-\infty$ and $\infty$ ) are inaccessible in finite expected time for $r$ (resp. $y$ ), the behavior of $u$ as $r$ and/or $y$ approach their inaccessible boundaries is implicitly determined by eq. (3).
} 
1. $\mathbb{E}\left\{\sup _{T \in\left[\tau, T_{+}\right]}\left[\frac{\partial \vartheta(T)}{\partial x}-\vartheta(T) \int_{\tau}^{T} \frac{\partial \vartheta(u)}{\partial x} d u\right]\right\}<\infty ;$

2. $\mathbb{E}\left\{\sup _{T \in\left[\tau, T_{+}\right]}\left[b_{(3), 11}(\tau)\left(\frac{\partial \vartheta(T)}{\partial x}-\vartheta(T) \int_{\tau}^{T} \frac{\partial \vartheta(u)}{\partial x} d u\right)\right]\right\}<\infty$ (and similarly for $b_{(1), 2}$ and $\sigma_{(1), 2}$ : see appendix A for the notation);

3. $\left.\mathbb{E}\left\{\sup _{T \in\left[\tau, T_{+}\right]}\left[\varphi_{(2), 1}(\tau)\right)\left(\int_{\tau}^{T} \frac{\partial \vartheta(u)}{\partial s} d u\right)\right]\right\}<\infty$;

4. $\mathbb{E}\left\{\sup _{T \in\left[\tau, T_{+}\right]}\left[\psi_{(2), 1}(\tau)\left(\left(\int_{\tau}^{T} \frac{\partial \vartheta(u)}{\partial s} d u\right)^{2}-\int_{\tau}^{T} \frac{\partial^{2} \vartheta(u)}{\partial s^{2}} d u\right)\right]\right\}<\infty$; and

5. $\left.\mathbb{E}\left\{\sup _{T \in\left[\tau, T_{+}\right]}\left[\sigma_{(1), 2}(\tau)\right)\left(\left(\int_{\tau}^{T} \frac{\partial \vartheta(u)}{\partial x} d u\right)^{2}-\int_{\tau}^{T} \frac{\partial^{2} \vartheta(u)}{\partial x^{2}} d u\right)\right]\right\}<\infty$.

These conditions are also very mild and are required to apply the Lebesgue's dominated convergence theorem when studying the local behavior of the pricing function (4): see, for instance, eq. (B4) in appendix B.

Eqs. (5) and (6) are convenient ways to represent partials of the bond price with respect to the short-term rate. Together with (3) and (4), these basic equations form the starting point of analysis of the paper.

When drift and diffusion terms of the volatility process in (1) do not depend on the short-term rate, the stochastic flow $\vartheta$ is always non-decreasing in the initial condition (use lemma A4 in appendix A for a rigorous proof of this): using the terminology of Bergman, Grundy and Wiener (1996), $\vartheta$ displays the "no-crossing" property. In this case, $u_{1}$ is always negative. Even when such a property is respected, there is no way to guarantee that the bond price is always strictly convex in the short-term rate: as (6) reveals, not only does $u_{11}$ depend on the first partial of $\vartheta$ with respect to the initial condition, it even depends on the mean curvature of the stochastic flow with respect to the initial condition. ${ }^{7}$

Next section characterizes the sign of $u_{11}$ in models without stochastic volatility. Apart from the trivial affine case, things are rather intricate. A general property I prove is that for bond prices to be strictly convex in the short-term rate, the curvature of the drift function under the risk-neutral measure must be sufficiently small (propositions 1 and 2). This is an interesting property since the last decade has witnessed a growing research interest in the empirical modeling of nonlinear drift functions (e.g., Aït-Sahalia (1996a), Conley et al. (1997) and Stanton (1997)).

\footnotetext{
${ }^{7}$ To illustrate with a simple example that the stochastic process describing the second partial of the solution of a stochastic differential equation with respect to the initial condition is not necessarily identically nil, consider the model of population growth in a stochastic crowded environment (e.g., Gard (1988, eq. (1.30) p. 166)):

$$
d r(\tau)=k_{1} r(\tau)\left(k_{2}-r(\tau)\right) d \tau+k_{3} r(\tau) d W(\tau), \tau \geq 0,
$$

where $k_{1}, k_{2}, k_{3}$ are given constants and the initial condition is $r(t)=x$. It can be verified (see chapter 4 , example 4.3 p. 116 in Gard (1988)) that the unique strong solution to this nonlinear stochastic differential equation is given by:

$$
r(\tau)=\frac{\exp \left\{\left(k_{1} k_{2}-\frac{1}{2} k_{3}^{2}\right) \tau+k_{3} W(\tau)\right\}}{x^{-1}+k_{1} \int_{t}^{\tau} \exp \left\{\left(k_{1} k_{2}-\frac{1}{2} k_{3}^{2}\right) s+k_{3} W(s)\right\} d s},
$$

which clearly validates the claim formulated above.
} 
In section 4 , I show that these basic properties extend quite easily to the stochastic volatility case when the volatility process evolves independently of the short-term rate (proposition 4). I am unable to obtain neat results on convexity issues when volatility also depends on the short-term rate. It is worth noticing that similar difficulties were pointed out by Romano and Touzi (1997, proposition 4.1 p. 408 and assumptions 4.1 and 4.2) in the stochastic volatility option pricing domain. Relating stochastic volatility to convexity of derivative prices with a high level of generality seems to be an unresolved issue at the moment.

\section{How do bond prices react to random volatility changes ? Introduc- tion}

A binomial example may illustrate very simply some aspects of the relationship between bond prices and volatility. Consider a risk-neutral tree in which the next period interest rate is either $i^{+}=i+d$ or $i^{+}=i-d$ with equal probability, where $i$ is the current interest rate level and $d>0$. The price of a two-period bond is $u(i, d)=m(i, d) /(1+i)$, where $m(i, d)=E\left\{1 /\left(1+i^{+}\right)\right\}$is the expected discount factor of the next period, which is greater than $1 /\left(1+E\left\{i^{+}\right\}\right)=1 /(1+i)$ due to Jensen's inequality: two-period bond prices increase with randomness. In fact, as shown in figure 1, two-period bond prices are always increasing in the "volatility" parameter $d$ in this example. Such a phenomenon can be connected with previous work by Jagannathan (1984, p. 429-430), who observed that in a two-period economy with identical underlying asset prices, a terminal underlying asset price $\widetilde{y}$ is a mean preserving spread of another terminal underlying asset price $\widetilde{x}$ (in the Rothschild and Stiglitz (1970) sense) if and only if the price of a call option on $\widetilde{y}$ is higher than the price of a call option on $\widetilde{x}$ : a key observation there was that if $\widetilde{y}$ is a mean preserving spread of $\widetilde{x}$, then $E(f(\widetilde{y}))>E(f(\widetilde{x}))$ for $f$ increasing and convex. ${ }^{8}$

The previous property is theoretically appealing, but it depends too much on the assumption that the expected short-term rate is independent of $d$. Consider indeed a multiplicative setting in which either $i^{+}=i(1+d)$ or $i^{+}=i /(1+d)$ with equal probability. Litterman, Scheinkman and Weiss (1991) showed that in such a setting, bond prices are decreasing in volatility at short maturity dates and increasing in volatility at long maturity dates. This is so because expected future interest rates increase over time at a strength positively related to $d$ : at short maturity dates, such an effect dominates the convexity effect illustrated in figure 1. At longer maturity dates, however, it is the convexity effect that dominates.

This paper offers a theoretically sound explanation of the previous and related phenomena. Consider for example the two-factor stochastic volatility model of the previous section, and differentiate eq. (3) with respect to volatility $(y)$. The result is that the first partial of the bond price with respect to volatility, $u_{2}(r, y, \tau, T)$, is the solution of

\footnotetext{
${ }^{8}$ To make such a connection more transparent in terms of the Rothschild and Stiglitz (1970) theory, let $\widetilde{m}_{d}\left(i^{+}\right)=1 /\left(1+i^{+}\right)$denote the random discount factor when $i^{+}=i \mp d$. Clearly $x \mapsto-\widetilde{m}_{d}(x)$ is increasing and concave, and so we must have: $E\left(-\widetilde{m}_{d^{\prime \prime}}(x)\right)<E\left(-\widetilde{m}_{d^{\prime}}(x)\right) \Leftrightarrow d^{\prime}<d^{\prime \prime}$, which is what demonstrated in figure 1 . In Jagannathan (1984), $f$ is increasing and convex, and so we must have: $E(f(\widetilde{y}))>E(f(\widetilde{x})) \Leftrightarrow \widetilde{y}$ is riskier than (or a mean preserving spread of) $\widetilde{x}$.
} 


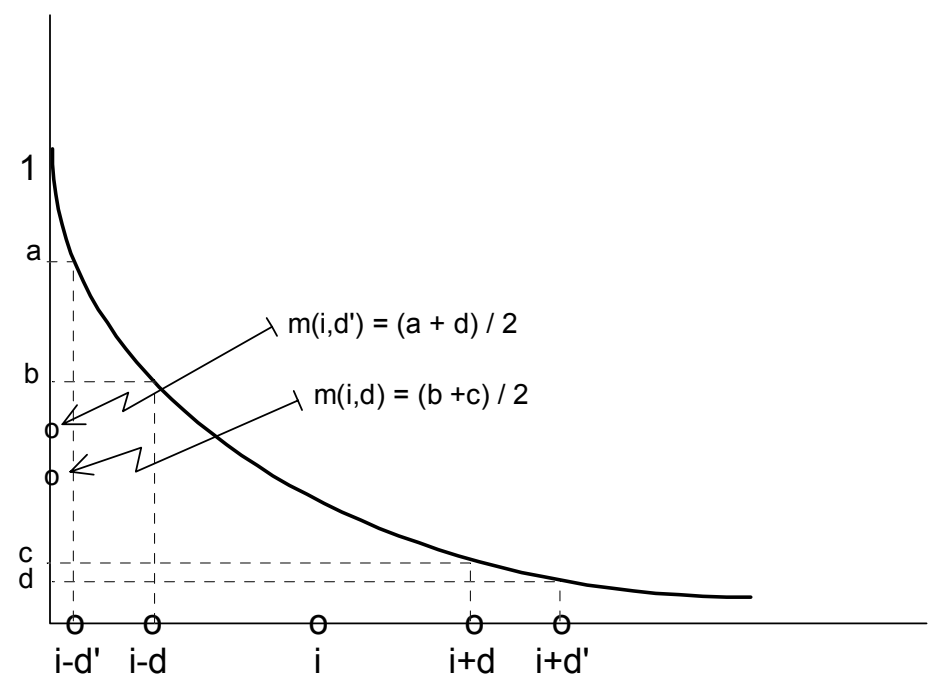

Figure 1 - A connection with the Rothschild-Stiglitz-Jagannathan theory: the simple case in which convexity of the discount factor induces bond prices to be increasing in volatility. If the risk-neutralized interest rate of the next period is either $i^{+}=i+d$ or $i^{+}=i-d$ with equal probability, the random discount factor $1 /\left(1+i^{+}\right)$is either $b$ or $c$ with equal probability. Hence $m(i, d)=$ $E\left\{1 /\left(1+i^{+}\right)\right\}$is the midpoint of $\overline{b c}$. Similarly, if volatility is $d^{\prime}>d, m\left(i, d^{\prime}\right)$ is the midpoint of $\overline{a d}$. Since $\overline{a b}>\overline{c d}$, it follows that $m\left(i, d^{\prime}\right)>m(i, d)$. Therefore, the two-period bond price $u(i, v)=m(i, v) /(1+i)$ satisfies: $u\left(i, d^{\prime}\right)>u(i, d)$ for $d^{\prime}>d$.

the following partial differential equation:

$$
\left\{\begin{array}{r}
0=\left(\frac{\partial}{\partial \tau}+L^{1}-k^{1}\right) u_{2}(r, y, \tau, T)+\left\{b_{2}(r, y) u_{1}(r, y, \tau, T)+\sigma_{2}(r, y) u_{11}(r, y, \tau, T)\right\} \\
\forall(r, y, \tau) \in \mathbb{R}_{++} \times \mathbb{R} \times[t, T) \\
u_{2}(r, y, T, T)=0, \forall(r, y) \in \mathbb{R}_{++} \times \mathbb{R}
\end{array}\right.
$$

where $L^{1}$ and $k^{1}$ play the same role as $L$ and $r$ in (3) (see appendix A for the precise definitions of $L^{1}$ and $k^{1}$ ). Consequently, by an application of the maximum principle (e.g., Friedman (1975)), one deduces that $u_{2}$ is always positive under the assumption that $b_{2} u_{1}+\sigma_{2} u_{11}$ is positive for each $(r, y, \tau) \in \mathbb{R}_{++} \times \mathbb{R} \times[t, T)$, thus confirming property VII) stated in the introduction. As regards the type of problems studied here, the maximum principle can be stated informally as follows: given a function $h$ with a constant sign for all $(r, y, \tau) \in \mathbb{R}_{++} \times \mathbb{R} \times[t, T]$, if another function $f$ satisfies $(\partial / \partial \tau+L-k) f+h=0$ for all $\tau \in[t, T)$ and $f=0$ at $T$ (as in eq. (7) above), then $f$ has the same sign as $h$ (see lemma A1 in appendix A for more technical details confirming the above conclusion). 
Arguably, the assumption that $b_{2} u_{1}+\sigma_{2} u_{11}$ is always positive is restrictive. By proposition 5 , for instance, $u_{1}$ is always negative at short maturity dates, even when volatility depends on the short-term rate; when $u_{11}>0, u_{2}$ is then positive at shortmaturity dates only with $b_{2}<0$. In fact, this conclusion is correct even when $u_{11}<$ 0 . Indeed, the sign of $b_{2} u_{1}+\sigma_{2} u_{11}$ is the result of a conflict between slope $\left(u_{1}\right)$ and convexity $\left(u_{11}\right)$ of the bond price but at short maturity dates, slope effects dominate convexity effects, i.e. $u_{11}$ tends to zero more rapidly than $u_{1}$ (see lemma A4 in appendix A); proposition 6 then establishes that bond prices are decreasing (resp. increasing) in volatility at short maturity dates when $b_{2}>0$ (resp. $\left.b_{2}<0\right)$. This clarifies property VIII) stated in the introduction and illustrates the previous multiplicative-tree model where: 1) expected future rates increase with $d$; and 2) the expected discount factor is decreasing in these rates. These two conditions correspond here to the conditions that 1) $b_{2}>0$, and 2) $u_{1}<0$ (which always holds at short maturity dates).

As is clear, volatility changes do not generally represent a mean preserving spread for the risk-neutral distribution in the term-structure domain. As originally pointed out by Jagannathan (1984), this is generally the case in the option pricing domain. In a stochastic volatility diffusion setting, for example, Romano and Touzi (1997, theorem 3.1 p. 406) confirmed this fact and showed that if an option price is strictly convex in the underlying stock price, then it is strictly increasing with respect to volatility. In the framework this paper analyzes, the short-term rate is not a traded asset. Therefore, the risk-neutral drift function $b$ is generally constrained to depend on volatility and as eq. (7) reveals, such a phenomenon generates slope effects. As shown in sections 4 and 5 , in the presence of a sufficiently high level of the market risk-premium, slope effects may even dominate at any finite maturity date, thus making bond prices decrease with volatility at any arbitrary maturity date. [Yet, in appendix G, a very specific counterexample is constructed in which convexity effects dominate at long maturity dates, thus making bond prices react positively to volatility changes at long maturity dates even when $b_{2}>0$.] Such phenomena also arise in thought-experiments taking place in scalar diffusion settings, and are described in the next section (see subsection 3.3) as a means to introduce the more complex theory of sections 4 and 5 .

\section{The scalar diffusion case}

\subsection{Slope and convexity properties}

As discussed in the previous section, slope and convexity properties play a quite important role in determining how bond prices react to volatility changes. The present subsection examines the restrictions that have to be respected by the short-term rate dynamics in order that bond prices display these properties in the scalar diffusion setting. (The scalar diffusion setup is obtained by setting $b(r, y) \equiv b(r)$ and $\sigma^{(1)}(r, y) \equiv \sqrt{2 a(r)}$, $\sigma^{(2)}(r, y) \equiv 0$ in $(1), \widehat{b}(r, y) \equiv \widehat{b}(r)$ and $\Lambda^{1}(r) \equiv \Lambda^{1}(r, y)$ in (2), and letting the price function in (3) be simply of the form $u(x, t, T)$.)

The following result shows that in the scalar diffusion setting, bond prices and the short-term rate are always negatively related. It also relates global convexity of the price function to the curvature of the drift function under the risk-neutral measure space. 
Proposition 1. Let assumption B1 in appendix B hold. Then, bond prices are always strictly decreasing in the short-term rate. Furthermore, if the risk-neutral drift of the short-term rate is at most such that

$$
\begin{gathered}
\max _{r \in \mathbb{R}_{++}} b^{\prime \prime}(r)<2, \\
\left(\text { resp. } \min _{r \in \mathbb{R}_{++}} b^{\prime \prime}(r)>2\right),
\end{gathered}
$$

then bond prices are strictly convex (resp. concave) in the short-term rate.

The conditions of the preceding proposition are easy to verify in models that do not place too many nonlinearities on the risk-neutral drift of the short-term rate. The following proposition contains less stringent conditions that are much easier to check in models with highly nonlinear drift functions (arising in nonparametric models, for instance).

Proposition 2. Let assumption B1 in appendix B hold. Then, for each current shortterm rate level $r(t)=x$ such that

$$
\begin{gathered}
-\infty<b^{\prime \prime}(x)<2 \\
\left(\text { resp. } 2<b^{\prime \prime}(x)<\infty\right),
\end{gathered}
$$

there exists a maturity date $\widehat{T}$ depending on $x$ such that bond prices are strictly convex (resp. concave) in the short-term rate at maturity dates less than $\widehat{T}$.

As it is well-known, bond prices are always a convex function of the short-term rate in affine models (i.e. models in which $b$ and $a$ are affine functions); the previous propositions clearly confirm this fact. The previous propositions can be used to check bond price convexity also in nonlinear models. Consider for example the model proposed by Ahn and Gao (1999). The authors take $\widehat{b}(r)=\beta_{0}\left(\beta_{1}-r\right) r$ and $\sqrt{2 a(r)}=\eta r^{3 / 2}$, and specify the risk premium function as: $\Lambda^{1}(r)=-\left(\lambda_{1} r^{-1 / 2}+\lambda_{2} r^{1 / 2}\right) / \eta$, which makes $b^{\prime \prime}(r)=-2\left(\beta_{0}+\lambda_{2}\right)$, all $r \in \mathbb{R}_{++}$. It can be shown that a condition for stationarity in this model is $\beta_{0}+\lambda_{2} \geq 0$, which is satisfied by the estimates reported by the authors (see their tables 3 and 5 ). Therefore, proposition 1 predicts that in this model, bond prices are strictly convex in the short-term rate.

In contrast, a nonlinear model not displaying the (global) convexity property is the one considered by Chapman, Long and Pearson (1999, p. 779-780). The authors take $\widehat{b}(r)=\beta_{0}+\beta_{1} r+\beta_{2} r^{2}+\beta_{3} r^{-1}, \sqrt{2 a(r)}=\eta r^{3 / 2}$, and $\sqrt{2 a(r)} \Lambda^{1}(r)=\lambda_{0} r^{3 / 2}+\lambda_{1} r^{5 / 2}+$ $\lambda_{2} r^{7 / 2}$. The drift specification is the one used by Ait-Sahalia (1996a) and Conley et al. (1997); and the diffusion specification is the one considered by Chan et al. (1992) and Conley et al. (1997), which is a special case of the diffusion function examined by Aït-Sahalia (1996a). For this model, I use the coefficient values reported by Chapman, Long and Pearson (1999), and apply the theoretical test conditions in proposition 2 to find that for all $r(t)=x<5.04 \%$ and for all $r(t)=x>27.23 \%$, there exist maturity dates for which bond prices are strictly concave in the short-term rate. 
The last example considered in this section is the "double square-root" model of Longstaff (1989). For technical reasons developed in appendix B, I consider a variant of this model that sets: $\widehat{b}(r)=\mu-\beta \sqrt{r}, \sqrt{2 a(r)}=\eta \sqrt{r}$, and $\Lambda=0$, with $\mu>\eta^{2} / 2$. Applying again proposition 2, I find that for all $r(t)=x<\beta^{2 / 3} / 4$, there exist maturity dates for which bond prices are strictly concave in the short-term rate.

\subsection{Comparison theory}

In the scalar diffusion case, it is also possible to use powerful comparison results (e.g., Karatzas and Shreve (1991, p. 291-295)) to relate very simply bond prices to the location of the short-term rate drift function. Consider two economies $A$ and $B$ in which the corresponding short-term rates $r^{A}$ and $r^{B}$ are solutions of:

$$
d r^{j}(\tau)=b^{j}\left(r^{j}(\tau)\right) d \tau+\sqrt{2 a\left(r^{j}(\tau)\right)} d W(\tau), r^{j}(t)=x^{j}, j=A, B,
$$

and suppose that $x^{A} \leq x^{B}$. Under the conditions given for instance in Karatzas and Shreve (1991, proposition 2.18 p. 293), one has that

$$
\operatorname{Pr}\left\{r^{A}(\tau) \leq r^{B}(\tau), \tau \in[t, \infty)\right\}=1,
$$

whenever the drift function in economy $A$ is less than the drift function in economy $B$, i.e. $b^{A}(r) \leq b^{B}(r), r \in \mathbb{R}_{++}$. Combining this result with the representation in (4) then reveals that bond prices in economy $A$ are higher than bond prices in economy $B .^{9}$

The above comparison results have an immediate economic interpretation. In the scalar diffusion setting, the thought-experiment of a permanent shift in the risk-neutral drift function can be interpreted as a permanent change of the unit risk-premium $\Lambda^{1}$ for the interest rate risk (see eqs. (2)). This means that if we had to visit two economies differing only in the amount of this risk-premium, we would observe a higher level of the yield curve in the more risk-premium demanding economy. I summarize:

Proposition 3. Let assumption A2 in appendix A hold. In the scalar diffusion setting, the term-structure of interest rates then increases with the risk-premium demanded by agents to be compensated for the stochastic fluctuations of the short-term rate, i.e., for any positive functions $\Lambda_{A}^{1}$ and $\Lambda_{B}^{1}$ satisfying the conditions of section 2, define $u\left(x, t, T ; \Lambda_{j}^{1}\right)$ as the solution of eq. (3) when $\Lambda^{1} \equiv \Lambda_{j}^{1}, j=A, B$; then $u\left(x, t, T ; \Lambda_{A}^{1}\right) \leq$ $u\left(x, t, T ; \Lambda_{B}^{1}\right)$ for all $T$ whenever $\Lambda_{A}^{1} \geq \Lambda_{B}^{1}$.

While the previous result may seem intuitive, it must be pointed out that it does not need to hold in more complicated diffusion settings (see section 7.1). Furthermore, it is surprising that no proof of it was available within the same general framework of this section. As an example, CIR (1985, p. 393) pointed out that bond prices go up

\footnotetext{
${ }^{9}$ It is possible to drop one restrictive condition given by Karatzas and Shreve (notably, a Lipschitz condition on one of the two $b^{j}$ ) at the expense of strengthening the condition $b^{A} \leq b^{B}$ to $b^{A}<b^{B}$. In this case, the same conclusions as above can be shown to hold (see Karatzas and Shreve (1991, exercise 2.19 p. 294)).
} 
when (minus) the market risk-premium goes down, but their observation concerned the specific case of their celebrated one-factor affine model. ${ }^{10}$

\subsection{Bond prices and volatility: comparative statics}

Elegant comparison results can not be used to implement comparative statics relating bond prices to volatility. Instead, the following approach is useful. Consider two economies $A$ and $B$ in which the corresponding short-term rates are solutions of:

$d r^{j}(\tau)=\left(\widehat{b}\left(r^{j}(\tau)\right)+\sqrt{2 a^{j}\left(r^{j}(\tau)\right)} \cdot \Lambda_{j}^{1}\left(r^{j}(\tau)\right)\right) d \tau+\sqrt{2 a^{j}\left(r^{j}(\tau)\right)} d W(\tau), r^{j}(t)=x, j=A, B$,

and suppose that for each $r \in \mathbb{R}_{++}, a^{A}(r)>a^{B}(r)$. The bond price functions are $u^{A}(r, \tau, T)$ in economy $A$ and $u^{B}(r, \tau, T)$ in economy $B$. For each $(r, \tau) \in \mathbb{R}_{++} \times[t, T)$, the price difference $\nabla u(r, \tau, T) \equiv u^{A}(r, \tau, T)-u^{B}(r, \tau, T)$ thus satisfies:

$$
\begin{aligned}
0 & =\frac{\partial}{\partial \tau} \nabla u+\left(\widehat{b}+\sqrt{2 a^{A}} \Lambda_{A}^{1}\right) \nabla u_{1}+a^{A} \nabla u_{11}-r \nabla u \\
& +\left\{\left(\sqrt{2 a^{A}}\left(\Lambda_{A}^{1}-\Lambda_{B}^{1}\right)+\left(\sqrt{2 a^{A}}-\sqrt{2 a^{B}}\right) \Lambda_{B}^{1}\right) u_{1}^{B}+\left(a^{A}-a^{B}\right) u_{11}^{B}\right\}
\end{aligned}
$$

with $\nabla u(r, T, T)=0 \forall r \in \mathbb{R}_{++}$. Next, suppose that the market risk-premium $\sqrt{2 a} \Lambda^{1}$ is positively valued and increases in volatility, i.e. $a^{A}(r)>a^{B}(r)$ all $r \in \mathbb{R}_{++} \Rightarrow$ $\left(\Lambda_{A}^{1}(r)-\Lambda_{B}^{1}(r)\right) / \Lambda_{B}^{1}(r)+\left(\sqrt{2 a^{A}(r)}-\sqrt{2 a^{B}(r)}\right) / \sqrt{2 a^{A}(r)}>0$ all $r \in \mathbb{R}_{++}$; note that most models, such as the CIR model, have $\Lambda_{A}^{1}=\Lambda_{B}^{1}$ and thus automatically fulfill the previous condition (see footnote 11 and appendix B). By proposition $1, u_{1}<0$. As mentioned in section $2, u_{1}$ is of higher order than $u_{11}$ at short maturity dates (see lemma $\mathrm{A} 4$ in appendix A). Hence, at short maturity dates, $\nabla u<0$ by a direct application of the maximum principle i.e., short-term bond prices are lower in the more volatile economy. ${ }^{11}$ In fact,

\footnotetext{
${ }^{10}$ The referee pointed out that in a thought-experiment in which the unit risk-premium changes, it is possible that the physical drift function also changes. I may illustrate such a remark with the help of the CIR example reported in footnote 11. There, $\Lambda(\cdot)=\epsilon \sqrt{r(\cdot)} / v$, where $\epsilon$ and $v$ are constants, and a change in $\epsilon$ (say) makes both the volatility and the physical drift functions change. Therefore, to apply the comparison results of this section, one has to think of a change in $\epsilon$, say, as one that is exactly counter-balanced by changes in other parameters (say $h, b$ or $k$ : see footnote 11) that keep volatility and physical drift unchanged. Without this kind of interpretation in mind, the comparative statics results of this section may only have a partial equilibrium flavor. The general case covered by proposition 3 is submitted to the same interpretation.

${ }^{11}$ CIR (1985, p. 393-394) state that bond prices are an increasing function of the volatility parameter in their single factor model because they define a market risk-premium $\sqrt{2 a} \Lambda \equiv \lambda r$ that is not literally taken to be proportional to the volatility parameter (see their eq. (22)). In terms of eq. (8), this implies that volatility affects bond prices only through convexity terms. Using the framework (and some notation) in Duffie's (1996, p. 230-233) textbook, however, one finds that a supporting equilibrium for the CIR model generates: $d r(\tau)=\left\{b v^{2}-(k \epsilon+\kappa) r(\tau)\right\} d \tau+k \cdot v \sqrt{r(\tau)} d W(\tau)$ and $\Lambda(\tau)=\epsilon \sqrt{r(\tau)} / v$, where $\epsilon<0$ (to ensure positive term-premia), $v \equiv \sqrt{h-\epsilon^{2}}$, and $b, h, \epsilon, \kappa, k$ are constants (a similar analysis can be conducted with the original CIR paper). Therefore, here a thought-experiment of an increase in the short-term volatility that is unambiguously interpreted only as a change in volatility (and not also as a change in the short-term rate drift under the physical measure) corresponds to a change in $k$ (in Duffie, $k$
} 
one can show that under fairly regular conditions, $\nabla u<0$ at any arbitrary maturity date. These conditions are developed in appendix B and impose a sufficiently high level of the risk-premium $\Lambda$. As checked there, the CIR model typically fulfills these conditions. Of course, in the extreme situation in which $\sqrt{2 a^{A}}\left(\Lambda_{A}^{1}-\Lambda_{B}^{1}\right)+\left(\sqrt{2 a^{A}}-\sqrt{2 a^{B}}\right) \Lambda_{B}^{1} \leq 0$ (which it does for example when the market risk-premium is not compensated), $\nabla u>0$ whenever bond prices are convex in the short-term rate.

\section{Models with stochastic volatility}

This section develops the central results of the paper. Its objective is to examine how random volatility changes affect bond prices dynamics. Section 4.1 provides results relating bond prices to short-term rate movements, and section 4.2 analyzes the relationship between bond prices and volatility.

\subsection{Bond prices and short-term rate movements}

The main objective of this subsection is to find conditions under which bond prices are strictly decreasing in the short-term rate. The following proposition contains results on slope and convexity issues when the volatility process in (1) does not depend too much on the short-term rate.

Proposition 4. Let assumption C1 in appendix $C$ hold. Then, if $\varphi_{1}(r, y)=\psi_{1}(r, y)=0$ for each $r, y \in \mathbb{R}_{++} \times \mathbb{R}$, then bond prices are strictly decreasing in the short-term rate. Suppose further that $\partial^{2} \sum_{j=1}^{2} \sigma^{(j)} \psi^{(j)}(r, y) / \partial r^{2}=0$. Then, bond prices are strictly convex (resp. concave) in the short-term rate if $\max _{r, y \in \mathbb{R}_{++} \times \mathbb{R}} b_{11}(r, y)<2$ (resp. $\left.\min _{r, y \in \mathbb{R}_{++} \times \mathbb{R}} b_{11}(r, y)>2\right)$. Alternatively, relax the previous assumptions; then for each $\forall(x, s) \in \mathbb{R}_{++} \times \mathbb{R}$ such that $-\infty<b_{11}(x, s)<2$ (resp. $\left.2<b_{11}(x, s)<\infty\right)$, there exists a maturity date $\widehat{T}$ depending on $x, s$ such that bond prices are always strictly convex (resp. concave) in the short-term rate at maturity dates less than $\widehat{T}$.

Stochastic volatility models not satisfying the conditions given in proposition 1 (i.e. $\varphi_{1}(\cdot, \cdot)=\psi_{1}(\cdot, \cdot)=0$ ) are much more complicated to analyze. Consider, for instance, the affine Longstaff and Schwartz (LS, henceforth) (1992) model. Such a model imposes that the process $(y(\tau) / r(\tau))_{\tau \geq 0}$ be in $(\alpha, \beta)$, and that the short-term rate and volatility satisfy:

$$
\left\{\begin{array}{l}
d r(\tau)=\left(b_{0}-b_{1} r(\tau)+b_{2} y(\tau)\right) d \tau+\alpha \sqrt{\frac{\beta r(\tau)-y(\tau)}{\alpha(\beta-\alpha)}} \cdot d W(\tau)+\beta \sqrt{\frac{y(\tau)-\alpha r(\tau)}{\beta(\beta-\alpha)}} \cdot d B(\tau) \\
d y(\tau)=\left(\varphi_{0}+\varphi_{1} r(\tau)-\varphi_{2} y(\tau)\right) d \tau+\alpha^{2} \sqrt{\frac{\beta r(\tau)-y(\tau)}{\alpha(\beta-\alpha)}} \cdot d W(\tau)+\beta^{2} \sqrt{\frac{y(\tau)-\alpha r(\tau)}{\beta(\beta-\alpha)}} \cdot d B(\tau)
\end{array}\right.
$$

where $\alpha>0, \beta>0, b_{j}$ and $\varphi_{j}$ are constants (see appendix $\mathrm{C}$ ). To fix ideas, assume that $\varphi_{1}<0$ (an analogous reasoning can be produced when $\varphi_{1}>0$ : see table 1), and

represents the volatility parameter of the primitive state process of the economy, i.e. the "shock" process affecting capital productivity). Note also that in this supporting equilibrium, a change in $k$ doesn't even make $\Lambda$ change. 
suppose that the short-term rate increases. Such an increase determines a decrease in future expected volatility via the $\varphi_{1}$-channel, and the important point to raise is to which extent such an effect makes bond prices increase. It may be the case, for instance, that bond prices are decreasing in volatility and that the effect on the term-structure induced by an increase of $x$ is offset by the negative feedback effect that $x$ has on the drift function of volatility.

The next result reveals that in the general model (1), bond prices display the reassuring and intuitive property to be strictly decreasing in the short-term rate at short maturity dates, even in the less favorable cases:

Proposition 5. Let assumption $C 1$ in appendix $C$ hold. Then, there exists a maturity date $T_{*}$ depending on the current short-term rate and volatility level $(r(t), y(t))=(x, s)$ such that bond prices are strictly decreasing in the short-term rate for each $T \leq T_{*}$.

The impossibility to exclude that bond prices are never increasing in the short-term rate in model (1) arises exactly because of the short-term rate feedbacks on the drift and volatility of the short-term rate volatility. As regards volatility drift feedbacks, for instance, the proof reveals that the negative feedbacks in the LS model are negligible at short maturity dates because volatility has a negligible impact on bond prices at short maturity dates. At longer maturity dates, such feedbacks may be important. To illustrate such a phenomenon in the context of the LS model, let $\varpi \equiv \partial \phi / \partial x$ denote the first partial of the stochastic volatility flow $\phi(\tau ; x, s)=y(\tau)$ with respect to the initial condition of the short-term rate $r(t)=x$. It can be shown that:

$$
\mathbb{E}(\varpi(\tau))=\frac{\varphi_{1}}{\bar{\theta}}\left\{\exp \left(-\theta_{-}(\tau-t)\right)-\exp \left(-\theta_{+}(\tau-t)\right)\right\}, \tau \in(t, T],
$$

where $\bar{\theta} \equiv \sqrt{\left(b_{1}-\varphi_{2}\right)^{2}+4 \varphi_{1} b_{2}}, \theta_{\mp} \equiv\left(b_{1}+\varphi_{2} \mp \bar{\theta}\right) / 2$. Next, suppose that $\theta_{-}>0$ (a stability condition ensured by the condition that $b_{1} \varphi_{2}>b_{2} \varphi_{1}$ ). Relation (10) then reveals that an increase in the short-term rate has no effect at $\tau=t$, yet as time unfolds, it has on average a progressively higher (negative) impact on volatility until time $\tau=t+\log \left(\theta_{+} / \theta_{-}\right)^{1 / \bar{\theta}}$, where function $\mathbb{E}(\varpi(\tau))$ attains its minimum. Now suppose that $\partial u / \partial y<0$ (see appendix $\mathrm{C}$ for numerical examples ensuring this). As illustrated in case (d) of table 1, bond prices might then be positively related to short-term rate movements at medium-long maturity dates. ${ }^{12}$ LS (1992) noticed that their model predicts that bond prices may react positively to short-term rate movements at medium-long maturity dates, and appendix $\mathrm{C}$ then provides further technical details on how to use the theory of this section to clarify the origins of this property.

\footnotetext{
${ }^{12}$ Since the LS model (9) is affine, the bond pricing function is always convex in the state variables. Furthermore, in model (9) the volatility of volatility is $\psi_{1} r+\psi_{2} y$, where $\psi_{1} \equiv-\alpha \beta(\alpha+\beta)$ (see appendix C). Therefore, case (d) in table 1 is the relevant case to refer to.
} 
Table 1

Cases (b) and (d) identify necessary conditions for bond prices to be positively related to the short-term rate at medium-long maturity dates when the volatility of volatility is decreasing in $r$ and bond prices are convex in volatility

risk-neutral drift of volatility

\begin{tabular}{|c|c|c|}
\hline & increasing in the short-term rate & decreasing in the short-term rate \\
\hline$\partial u / \partial y>0$ & (b) & (a) \\
\hline$\partial u / \partial y<0$ & (c) & (d) \\
\hline
\end{tabular}

\subsection{Bond prices and volatility}

The objective of this subsection is to examine the mechanism generating given relationships between bond prices and volatility. As indicated in section 2, a sufficient condition under which bond prices are increasing in volatility at any finite maturity date is:

$$
\text { For all }(r, y, \tau) \in \mathbb{R}_{++} \times \mathbb{R} \times[t, T), \quad b_{2}(r, y) u_{1}(r, y, \tau, T)+\sigma_{2}(r, y) u_{11}(r, y, \tau, T)>0 \text {. }
$$

As an example, LS (1992, table II p. 1278) reported parameter estimates of their model (9) guaranteeing that $u_{1}<0$. The authors also reported a negative estimate of $b_{2}$. Similar findings were reported by Chapman, Long and Pearson (1999, p. 800-801). Since (9) is an affine model, $u_{11}>0$. Because $\sigma(r, y)=y / 2$ in (9), condition (11) then implies that given this kind of parameter estimates, bond prices can never be decreasing in volatility in the LS model.

When $b_{2}>0$, the situation is radically different. Such a situation may obviously arise within the LS model (9) under a set of alternative parameter values and in general, it arises whenever the interest rate risk premium $\sum_{j=1}^{2} \sigma^{(j)}(r, y) \cdot \Lambda^{j}(r, y)$ is positively valued and increases sufficiently rapidly with volatility (see eqs. (2)). If $\widehat{b}_{2} \equiv 0$, the condition that $b_{2}>0$ is automatically satisfied whenever the interest rate risk-premium is increasing in volatility. Table 2 describes three models that have $\widehat{b}_{2} \equiv 0$ (FV: Fong and Vasicek (1991); AL: Andersen and Lund (1997b); MF: Mele and Fornari (2000)).

Table 2

Examples of stochastic volatility models in which the risk-neutralized drift function of the short-term rate is increasing in volatility

\begin{tabular}{ccccccc} 
& $b(r, y)$ & $\sigma^{(1)}(r, y)$ & $\sigma^{(2)}(r, y)$ & $\varphi(r, y)$ & $\psi^{(1)}(r, y)$ & $\psi^{(2)}(r, y)$ \\
\hline \hline $\mathrm{FV}$ & $\iota-\theta r+\lambda_{1} y$ & $\sqrt{y}$ & 0 & $w-\left(\varphi_{2}+\lambda_{2} \psi_{0}\right) y$ & $\rho \psi_{0} \sqrt{y}$ & $\psi_{0} \sqrt{y} \sqrt{1-\rho^{2}}$ \\
$\mathrm{AL}$ & $\iota-\theta r+\lambda_{1} e^{y / 2} r$ & $e^{y / 2} r^{\gamma}$ & 0 & $w-\varphi_{2} y$ & $\psi_{0}$ & 0 \\
$\mathrm{MF}$ & $\iota-\theta r+\lambda_{1} y^{1 / \delta} r$ & $y^{1 / \delta} \sqrt{r}$ & 0 & $w-\varphi_{2} y-\lambda_{2} \psi_{0} y \sqrt{r}$ & $\psi_{0} y$ & 0
\end{tabular}

In these models, $\lambda_{1}$ and $\lambda_{2}$ are risk-premia coefficients, $\iota, \theta, \gamma, \delta, w, \varphi_{2}, \psi_{0}$ are constants and $\delta \in[1, \infty), \theta \in(0, \infty), \rho \in(-1,+1)$. The constant $\lambda_{1}$ is typically found to be positive 
to accommodate main stylized features of the entire term-structure of interest rates, at least in the context of models with zero correlation. In the context of three-factor models with nonzero correlations, Dai and Singleton (2000) provided mixed empirical evidence on the sign of such coefficient, which is discussed and used to illustrate the multifactor theory of the next section. The following proposition provides a theory on how bond prices react to random volatility changes in models that may make $b_{2}>0$, independently of the sign of $\sigma_{2}$.

Proposition 6 ("Weak" term-structure augmenting (decreasing) volatility property). Let assumption C2 in appendix $C$ hold. Then, for each current short-term rate and volatility level $(r(t), y(t))=(x, s) \in \mathbb{R}_{++} \times \mathbb{R}$ such that $b_{2}(x, s) \in(0, \infty)\left(\right.$ resp. $b_{2}(x, s) \in$ $(-\infty, 0)$ ) and $0<\left|\sigma_{2}(x, s)\right|<\infty$, there exists a maturity date $\bar{T}$ (resp. $\underline{T}$ ) depending on $(x, s)$ such that bond prices are always decreasing (resp. increasing) in volatility for all maturity dates less than $\bar{T}$ (resp. $\underline{T})$.

When do bond prices satisfy a sort of "strong" form of the term-structure augmenting (decreasing) volatility property? The answer clearly depends on whether condition (11) is satisfied, and is model-specific. Under fairly regular conditions, however, all the model examples of this section predict that bond prices may be decreasing in volatility at any arbitrary maturity date in correspondence of sufficiently high levels of the interest rate risk-premium (the case of bond prices increasing in volatility has a similar interpretation). ${ }^{13}$ This is the case, for instance, of the LS model (9) (see appendix C for details). Also, it can be shown that the Feynman-Kac stochastic representation of $u_{2}$ in the Andersen and Lund (1997) model of table 2 is (the model of Mele and Fornari (2000) can be analyzed similarly):

$$
\begin{aligned}
u_{2}(x, s, t, T)= & \frac{1}{2} \mathbb{E}\left\{\int_{t}^{T} \kappa^{1}(t, \tau)\left(-u_{1}\left(\vartheta_{(1)}(\tau), \phi_{(1)}(\tau), \tau, T\right)\right) \exp \left(\phi_{(1)}(\tau)\right) \vartheta_{(1)}(\tau)^{2 \gamma}\right. \\
& \left.\left.\times\left(\frac{u_{11}}{-u_{1}}\left(\vartheta_{(1)}(\tau), \phi_{(1)}(\tau), \tau, T\right)-\lambda_{1} \exp \left(-\frac{1}{2} \phi_{(1)}(\tau)\right) \vartheta_{(1)}(\tau)^{1-2 \gamma}\right) d \tau\right)\right\},
\end{aligned}
$$

where $\kappa^{1}, \vartheta_{(1)}, \phi_{(1)}$ are as in appendix A, lemma A3. Under the conditions introduced in appendix $\mathrm{B}$ for a related problem (see (C4)), a fixed point argument can then be employed (as in (C5)) to ensure that there exist sufficiently high values of $\lambda_{1}$ depending on $T, x, s$ that make $u_{2}<0$ for any finite $T .{ }^{14}$ In numerical work, AL found that $u_{2}<0$ for all maturity dates up to thirty years. In numerical work, Andersen and Lund set $\lambda_{1}=7$, and found that $u_{2}<0$ for any maturity dates up to thirty years (similar results

\footnotetext{
${ }^{13}$ Conditions and methods of proofs are similar to the ones developed for the scalar diffusion case (see appendix B).

${ }^{14}$ Alternatively, one may wish to explore the idea to let $\lambda_{1}$ increase while keeping the double integral of $-u_{11} / u_{1}$ constant by changing smoothly at the same time the remaining parameters of the model (e.g., $\theta)$. Specifically, conditions such as (C4) in appendix $\mathrm{C}$ will be satisfied whenever there is one parameter with an effect on $-u_{11} / u_{1}$ which is the opposite of the effect of $\lambda_{1}$ on $-u_{11} / u_{1}$. A fixed point argument of the kind of eq. (C5) can then always be made.
} 
were reported by Mele and Fornari (2000)). ${ }^{15}$ The authors attribute this finding to 1) the presence of a positively priced risk-premium; and 2) high mean-reversion in volatility. As demonstrated here, the first conjecture of the authors is correct.

Finally, consider the Fong and Vasicek (1991) model. In this model, it is possible to analytically find uniform bounds for the critical maturity date $\bar{T}$ of proposition 6 . Let $\lambda_{1}>0$; in appendix C, I show that:

$$
\bar{T}=\left\{\begin{array}{l}
t-\log \left(1-2 \theta \lambda_{1}\right)^{1 / \theta}, \text { if } \lambda_{1} \in\left(0, \frac{1}{2 \theta}\right) \\
\text { any strictly positive real number, otherwise }
\end{array}\right.
$$

Relations (12) say that the persistence of the term-structure augmenting volatility property increases with the risk-premium coefficient $\lambda_{1}$. They also reveal that with a sufficiently high interest rate risk-premium (viz $\lambda_{1} \geq 1 / 2 \theta$ ), positive volatility changes make the term-structure of interest rates increase at any arbitrary finite maturity date. Relations (12) also show that according to this model, it is the persistence of the short-term rate process that plays an important role in explaining the term-structure augmenting volatility property, not the persistence of the volatility process.

\section{Stochastic central tendency models in a three-factor setting}

The original purpose of models with a stochastic central tendency (e.g. Andersen and Lund (1997b), Chen (1996) and Balduzzi et al. (1996)) was to make the short-term rate revert towards a stochastically moving long-term value. More recently, Dai and Singleton (2000) produced empirical evidence that within the class of affine models, US historical interest rates behavior can only be adequately represented by models with a rich feedback structure between the state variables and with correlated Brownian motions. An example of such models is:

$$
\left\{\begin{aligned}
& d r(\tau)=\{\kappa(\ell(\tau)-r(\tau))-\left.\left(\lambda_{r}+\lambda_{v} \sigma_{r v} \eta^{2}\right) y(\tau)-\lambda_{\theta} \sigma_{r \theta} \zeta^{2}\right\} d \tau \\
&+\sqrt{y(\tau)} d W(\tau)+\sigma_{r v} \eta \sqrt{y(\tau)} d B(\tau)+\sigma_{r \theta} \zeta d Z(\tau) \\
& d y(\tau)=\left\{\mu \bar{v}-\left(\mu+\lambda_{v} \eta^{2}\right) y(\tau)\right\} d \tau+\eta \sqrt{y(\tau)} d B(\tau) \\
& d \ell(\tau)=\left\{\nu(\bar{\theta}-\ell(\tau))-\lambda_{\theta} \zeta^{2}-\lambda_{r} \sigma_{\theta r} y(\tau)\right\} d \tau+\sigma_{\theta r} \sqrt{y(\tau)} d W(\tau)+\zeta d Z(\tau)
\end{aligned}\right.
$$

where $W, B, Z$ are independent Brownian motions under the risk-neutral measure, and the notation for the various constants is the one used by the authors, with $\lambda_{r}, \lambda_{v}, \lambda_{\theta}$ denoting risk-premia coefficients.

\footnotetext{
${ }^{15}$ In fact, the numerical exercises reported by Andersen and Lund (1997b) that are of interest here concern a three-factor model of the kind analyzed in the following section. It is possible to show, however, that the analysis of this section remains the same even in the three-factor model considered by Andersen and Lund (1997b).
} 
In this section, I generalize both model (1) and (13) and take as primitive:

$$
\left(\begin{array}{l}
d r(\tau) \\
d y(\tau) \\
d \ell(\tau)
\end{array}\right)=\left(\begin{array}{l}
b(r(\tau), y(\tau), \ell(\tau)) \\
\varphi(r(\tau), y(\tau), \ell(\tau)) \\
\varepsilon(r(\tau), y(\tau), \ell(\tau))
\end{array}\right) d \tau+V(r(\tau), y(\tau), \ell(\tau))\left(\begin{array}{c}
d W(\tau) \\
d B(\tau) \\
d Z(\tau)
\end{array}\right), \text { for } \tau \in(t, T]
$$

where $V$ is a $3 \times 3$ matrix with $[V]_{1 j}(r, y, \ell) \equiv \sigma^{(j)}(r, y, \ell),[V]_{2 j}(r, y, \ell) \equiv \psi^{(j)}(r, y, \ell)$, and $[V]_{3 j}(r, y, \ell) \equiv \pi^{(j)}(r, y, \ell), j=1,2,3 ;(r(t), y(t), \ell(t))=(x, s, c)$, and the various drift and diffusion coefficients satisfy the same conditions as those of system (1) (the riskpremia are defined similarly as in eq. (2)); finally, I set $\sigma(r, y, \ell) \equiv \sum_{j=1}^{3} \sigma^{(j)}(r, y, \ell)^{2} / 2$, $\psi(r, y, \ell) \equiv \sum_{j=1}^{3} \psi^{(j)}(r, y, \ell)^{2} / 2$ and $\pi(r, y, \ell)=\sum_{j=1}^{3} \pi^{(j)}(r, y, \ell)^{2} / 2$.

Naturally, it is generally impossible to interpret one of the unobserved factors as "stochastic volatility" in system (14). This would be possible when, say, $\partial \sigma / \partial \ell=0$ and $\partial \sigma / \partial y>0$, in which case only factor $y$ can be interpreted as a stochastic volatility factor, as in model (13) and in model (16) below. Such interpretative (and arbitrary) constraints, however, will not be imposed to derive propositions 7 and 8 in the present section.

Finally, note that I will omit to state the regularity conditions under which the results of this section hold because these represent a straighforward generalization of the conditions stated for the previous sections.

We have:

Proposition 7 (Weak term-structure augmenting (decreasing) unobservable factor property). For each current factor level $(r(t), y(t), \ell(t))=(x, s, c) \in \mathbb{R}_{++} \times \mathbb{R} \times \mathbb{R}$ such that $b_{i}(x, s, c) \in(0, \infty)\left(\right.$ resp. $\left.b_{i}(x, s, c) \in(-\infty, 0)\right)$ and $\left|\sigma_{i}(x, s, c)\right| \in(0, \infty), i=2,3$, there exists a maturity date $\bar{T}($ resp. $\underline{T})$ depending on $(x, s, c)$ such that bond prices are always decreasing (resp. increasing) in factor $j, j=y, \ell$, for all maturity dates less than $\bar{T}$ $(\operatorname{resp} . \underline{T})$.

The previous result generalizes proposition 6 , and is due to phenomena very similar to those mentioned in section 2: precisely, the bond price reaction behavior at short maturity dates is still led by slope effects (see lemma D1 and eq. (D2)). However, the conditions guaranteeing the existence of a strong version of proposition 7 are more complex than conditions (11): 
Proposition 8 (Strong term-structure augmenting (decreasing) unobservable factor property). For any $T>t$, bond prices are decreasing (resp. increasing) in factor $j$, $j=y, \ell$, for all maturity dates up to $T$ if for all $(r, y, \ell, \tau) \in \mathbb{R}_{++} \times \mathbb{R} \times \mathbb{R} \times[t, T)$,

$$
\begin{aligned}
& \left.\frac{\partial b}{\partial y} \frac{\partial u}{\partial r}+\frac{\partial \sigma}{\partial y} \frac{\partial^{2} u}{\partial r^{2}}+\frac{\partial \varepsilon}{\partial y} \frac{\partial u}{\partial \ell}+\frac{\partial \pi}{\partial y} \frac{\partial^{2} u}{\partial \ell^{2}}+\frac{\partial^{2} u}{\partial r \partial \ell} \cdot \sum_{j=1}^{3} \frac{\partial}{\partial y} \sigma^{(j)} \pi^{(j)}<0 \text { (resp. }>0\right)(\text { factor } y) \\
& \frac{\partial b}{\partial \ell} \frac{\partial u}{\partial r}+\frac{\partial \sigma}{\partial \ell} \frac{\partial^{2} u}{\partial r^{2}}+\frac{\partial \varphi}{\partial \ell} \frac{\partial u}{\partial y}+\frac{\partial \psi}{\partial \ell} \frac{\partial^{2} u}{\partial y^{2}}+\frac{\partial^{2} u}{\partial r \partial y} \cdot \sum_{j=1}^{3} \frac{\partial}{\partial \ell} \sigma^{(j)} \psi^{(j)}<0(\text { resp. }>0)(\text { factor } \ell)
\end{aligned}
$$

Comparing the first condition in (15) with (11) reveals that the new terms arising from the presence of a third factor are: 1) slope and convexity of bond prices with respect to factor $\ell$; and 2) correlation terms. As shown in the examples below, these new terms may be important to explain given relationships between bond prices and factor $y$ (the analysis for factor $\ell$ is identical).

An example of models that can be analyzed quite easily with the help of conditions (15) is one proposed in Dai and Singleton (2000):

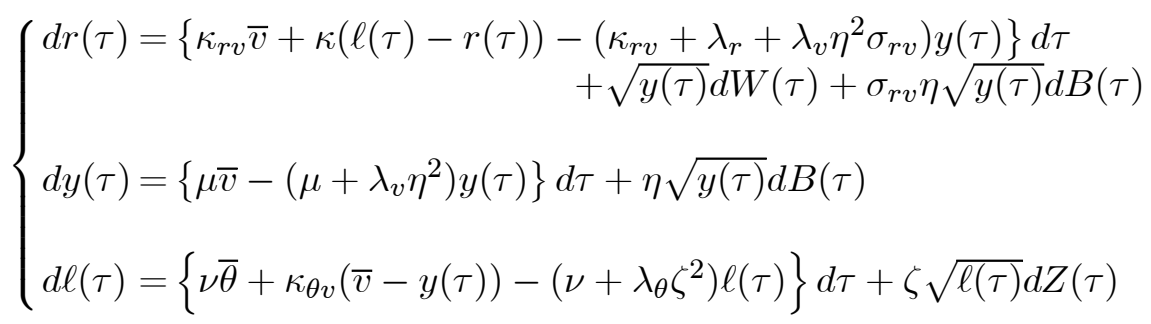

The authors reported a negative estimate of $-b_{2} \equiv \kappa_{r v}+\lambda_{r}+\lambda_{v} \sigma_{r v} \eta^{2}$ (see their table III p. 1965). Therefore, proposition 7 predicts that in model (16), bond prices are negatively related to changes in both factors $y$ and $\ell$ at short maturity dates. In addition, in appendix D, I show that when $\kappa>0$ and $\kappa_{\theta v}<0$ (as in the estimates reported by the authors), proposition 8 then predicts that a sufficient condition for bond prices to be decreasing in volatility at any finite maturity date is that:

$$
\frac{-2\left(\kappa_{r v}+\lambda_{r}+\lambda_{v} \sigma_{r v} \eta^{2}\right)}{1+\eta^{2} \sigma_{r v}^{2}}>\frac{1}{\kappa}
$$

which is amply satisfied by the estimates reported by the authors. Conditions (17) generalize conditions (12) exactly because they keep track of the rich feedback and correlation structure of model (16): only when $\kappa_{r v}=\sigma_{r v}=0$ do conditions (17) reduce to (12). Also in this model, bond prices are negatively related to changes in factor $\ell$ at any finite maturity date (see appendix D). ${ }^{16}$

An issue that deserves a special mention here is that the factor $\ell$ dynamics may be important in explaining the sign of the l.h.s. of (15). This is not so evident in model

\footnotetext{
${ }^{16}$ Given the negative value of $\nu+\lambda_{\theta} \zeta^{2}$ implied by the estimates reported by the authors, the solution of (16) is defined only up to an explosion time.
} 
(16) and condition (17) (see, however, eq. (D7) in appendix D for more details on this), but can be clearly seen at work within model (13). Precisely, Dai and Singleton (2000, table II p. 1964) reported a positive estimate of $-b_{2} \equiv \lambda_{r}+\lambda_{v} \sigma_{r v} \eta^{2}$ in model (13), and according to proposition 7 , bond prices are increasing in volatility at short-maturity dates. Furthermore, in appendix D, I show that if $\lambda_{r}+\lambda_{v} \sigma_{r v} \eta^{2}>0, \lambda_{r} \sigma_{\theta r}<0$ and $\kappa>\nu$ (as in the estimates reported by the authors), then proposition 8 predicts that bond prices are increasing in volatility at any finite maturity date whenever:

$$
\frac{\lambda_{r}+\lambda_{v} \sigma_{r v} \eta^{2}}{-\lambda_{r} \sigma_{\theta r}}>\frac{\kappa}{\nu}
$$

Due to the high value of the ratio $\kappa / \nu$ estimated by the authors, however, condition (18) is not satisfied. Intuitively, given $\nu$, a high $\kappa$ means that the third term in (15) is also high in absolute value. This is so because $\left.(\partial \varepsilon / \partial y) \cdot(\partial u / \partial \ell)=-(\partial \varepsilon / \partial y) \cdot \int m_{\vartheta, c}(v) d v\right) \cdot u$, where $m_{\vartheta, c}(v)$ is the partial of the short-term rate $\vartheta$ with respect to the initial condition $c$ of factor $\ell$; and in the model estimated by Dai and Singleton, $m_{\vartheta, c}$ is positive and increases with $\kappa$ (see appendix D). In fact, in appendix D, I use condition (15) to show that bond prices are positively related to volatility up to eight-ten months. By elaborating further condition (15), however, I also provide another condition for bond prices to be decreasing in volatility at longer maturity dates, and I find that such a condition is satisfied at least for maturity dates of two years.

Back to the general theory, the final remark of this section is that the bond price reaction to short-term rate movements is governed by the same logic presented in section 5. Particularly, results similar to propositions 4 and 5 also apply here (see the proof of proposition 7; as regards proposition 4, the appropriate condition is that the coefficients of $y$ and $\ell$ do not depend on $r$ ). As regards the Dai and Singleton (2000) models (13) and (16), for instance, bond prices are always decreasing in $r$.

\section{On jumps and default}

This section examines the robustness of the theory developed in the previous sections to the introduction of jump phenomena. I shall consider two settings: jump-diffusion models for the short-term rate, and models of defaultable bonds. In both cases, jumps will be modeled as being governed by Poisson processes. Jump-diffusion models for the short-term rate have traditionally attracted the interest of modelers because they may capture sudden changes in the market liquidity conditions and/or discontinuous information releases. A first general equilibrium treatment of this kind of models appears in Ahn and Thompson (1988); a very detailed list of references on recent empirical work in this area can be found in Das (2000). As regards defaultable bonds, I shall make reference to "reduced form" models (see, for instance, Duffie and Singleton (1999) and the references therein), in which default is considered as an exogenously given rare event.

One important reason for which jumps may be important in qualifying some of the properties of the previous sections lies in the possibility of the violation of the property that bond prices movements are negatively related to short-term rate movements. Let us see how with a very simple example adapted from Karlin and Taylor (1981, p. 258-260). Figure 2 depicts sample trajectories of a positive valued process that exhibits continuous 


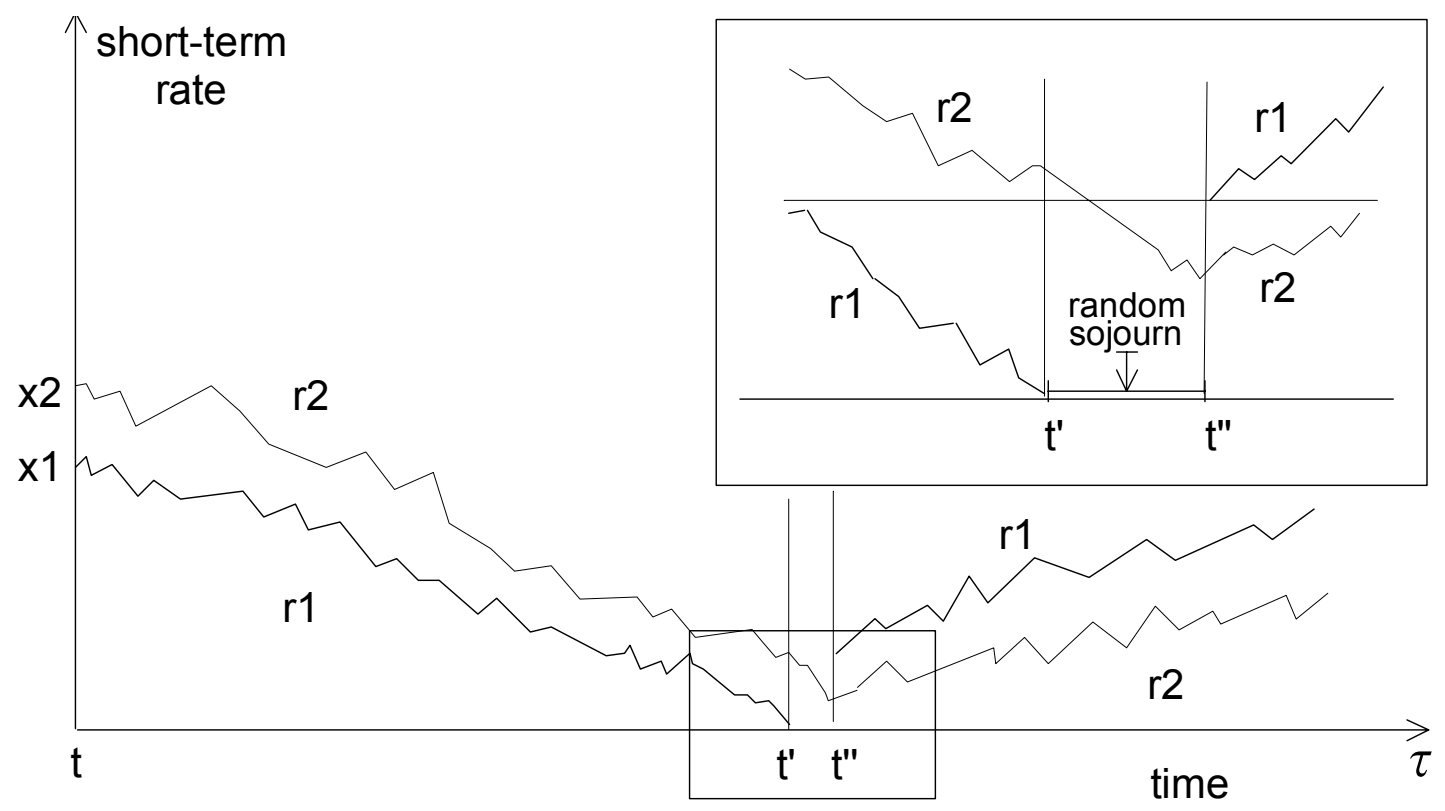

Figure 2 - Violation of the no-crossing property: a jump discontinuity at zero. The two lines depict sample trajectories of the short-term rate from the stochastic differential equation: $d r(\tau)=b(r(\tau)) d \tau+\sqrt{2 a(r(\tau))} d W(\tau)$ for a given realization of the Brownian motion. In this example, it is assumed that zero is attainable in finite expected time (i.e., it is a regular boundary point). If zero is reached, the process $r$ waits there for a random duration, and then jumps to the strictly positive region, where its stochastic evolution will be governed by the same stochastic differential equation as before. The solid line (labeled $r_{1}$ ) retraces a sample trajectory of this process that starts at $x_{1}$, reaches zero for the first time at $\tau=t^{\prime}$ and leaves the boundary at the random departure time $\tau=t^{\prime \prime}$. The dashed line (labeled $r_{2}$ ) depicts the trajectory of the short-term rate from the same stochastic differential equation while keeping fixed the same realization of the Brownian motion $W$, but with $r(t)=x_{2}>x_{1}$. During the sojourn at the boundary, $r_{1}$ has been governed by a probabilistic scheme that is different from the probabilistic scheme of the strictly positive region, while during such a sojourn of $r_{1}, r_{2}$ has still evolved as $d r_{2}(\tau)=b\left(r_{2}(\tau)\right) d \tau+\sqrt{2 a\left(r_{2}(\tau)\right)} d W(\tau)$. We can always find a trajectory of $W$ such that $r_{2}$ becomes less than $r_{1}$, as illustrated in this figure, and the no-crossing property is violated. The reason for the violation of the no-crossing property is that in the period from $t^{\prime}$ to $t^{\prime \prime}$, no information about $W(\tau)$ is transmitted to $r_{1}$, while this is not the case for $r_{2}$.

sample paths over the interior of the state-space but not at the boundary. At the boundary, the process can wait for a random duration that has an exponential distribution, and then jump to the interior of the state-space according to a given distribution. As shown by Karlin and Taylor (1981, formula 8.20 p. 259), such a process can be Markov only when the sample paths are allowed to be discontinuous. But this contradicts the 
no-crossing property, thus implying that bond prices now need not be negatively related to short-term rate changes at all maturity dates.

As is clear, this example is not an obscure pathology of the no-crossing property. On the contrary, it emphasizes how important is the assumption that the short-term rate be a diffusion process (i.e., a Markov process with continuous sample paths) to recover the property that bond prices are decreasing in the short-term rate. In this section, however, I will show that under suitable restrictions on the various hazard functions governing jumps, the properties of the previous sections remain essentially unaffected.

\subsection{Jump-diffusion models}

Let the short-term rate be solution of

$$
d r(\tau)=b^{J}(r(\tau)) d \tau+\sqrt{2 a_{1}(r(\tau))} d W(\tau)+a_{2}(r(\tau)) \cdot \mathcal{S} \cdot d N(\tau), \tau \in(t, T], r(t)=x,
$$

where $b^{J}$ is the jump-adjusted risk-neutral drift, $a_{1}$ is a strictly positive "diffusion" function, $a_{2}$ is a bounded "jump" function with bounded derivatives and $N$ is a Cox process with intensity function (or "hazard rate") that for simplicity I take to be of the form $v(r)$, where $v$ is bounded with bounded derivatives. Finally, $\mathcal{S}$ is a random variable with a fixed probability measure on $\mathbb{R}$ with density $p$ and expectation operator $\mathbb{E}_{\mathcal{S}}$. [See, e.g., Jacod and Shiryaev (1987, p. 142-146) for a succinct discussion of diffusion processes with jumps.]

We have:

Proposition 9 Let the short-term rate be solution of (19). The following statements are true:

a) There exists a maturity date $T_{*}$ depending on the current short-term rate level $r(t)=x$ such that bond prices are strictly decreasing in $x$ for each maturity date $T \leq T_{*}$.

b) Assume that for all $r \in \mathbb{R}_{++}, a_{2}^{\prime}(r)=0$. Then, bond prices are always decreasing in the short-term rate if for all $r \in \mathbb{R}_{++},-1 \leq v^{\prime}(r) \leq 0$; when for all $r \in \mathbb{R}_{++}, v^{\prime}(r)>0$, bond prices are always decreasing in the short-term rate if for each $(r, \tau) \in \mathbb{R}_{++} \times[t, T)$, $v^{\prime}(r) /\left(1+v^{\prime}(r)\right)<u(r, \tau, T) / \mathbb{E}_{\mathcal{S}}\left\{u\left(r+a_{2} \mathcal{S}, \tau, T\right)\right\}$, where $a_{2}$ is a constant.

c) Assume that for all $r \in \mathbb{R}_{++}, a_{2}^{\prime}(r)=0$ and $v^{\prime}(r)=0$. Then, the conclusions about bond price (global) convexity of proposition 1 (applied to $b^{J}$ ) are also valid here.

d) Consider two economies $A$ and $B$ which only differ because for each $r \in \mathbb{R}_{++}$, $v^{A}(r)>v^{B}(r)$ and let $u^{j}, j=A, B$, be the corresponding bond price functions. Finally, assume that $b^{J}$ does not depend on $v$. Then, for each $(r, \tau) \in \mathbb{R}_{++} \times[t, T), u^{A}(r, \tau, T)<$ $($ resp. $>) u^{B}(r, \tau, T)$ whenever $\mathbb{E}_{\mathcal{S}}\left\{u^{B}\left(r+a_{2}(r) \mathcal{S}, \tau, T\right)\right\}<($ resp. $>) u^{B}(r, \tau, T)$.

Under the same conditions of prop. 9-c), the local convexity properties of prop. 2 still hold here (with respect to $b^{J}$ ) for sets of bond prices satisfying the standard regularity condition that $\partial u_{1}(x, t, T) /\left.\partial T\right|_{T=t}=-1$. As regards multifactor models, the analysis of sections 4 and 5 is unaffected as long as intensity and jump functions are independent of the state variables [by a simple extension of eq. (E1) in appendix E to the multidimensional case; prop. 9-a) will then hold even when intensity and jump functions depend on factors levels, and local analysis is unchanged for all bond prices 
satisfying standard regularity conditions as: $\left.\partial u_{11}(x, s, t, T) /\left.\partial T\right|_{T=t}=0\right]$. When the hazard rates do depend on the state variables, in some cases (not reported here for brevity) the analysis can be conducted similarly as in the proof of prop. 9-b).

\subsection{Defaultable bonds}

Assume that under a risk-neutral measure, the short-term rate $(r(\tau))_{\tau \in[t, T]}$ is a diffusion process and that the event of default at each instant of time is exactly the same as process $N$ considered in the previous subsection (with intensity process $v$ ). In case of default at point $\tau$, the holder of the bond receives a recovery payment $\bar{u}$ that I assume to be of the form $\bar{u}(\tau) \equiv \bar{u}(\tau, r(\tau))$. Let the pre-default bond price function be $u^{\operatorname{pre}}(r, \tau, T)$, and set $\bar{u}=(1-l) \cdot u^{\text {pre }}$ for some process $l$ in $[0,1]$. Let $\mathbb{E}^{*}\{\cdot\}$ be the expectation operator taken with reference to the information sets $\sigma(r(u): t \leq u \leq \tau)$ only. As shown by Duffie and Singleton (1999, eq. (10) p. 696),

$$
u^{\mathrm{pre}}(x, t, T)=\mathbb{E}^{*}\left\{\exp \left(-\int_{t}^{T}(r(\tau)+l(\tau) \cdot v(r(\tau))) d \tau\right)\right\},
$$

and in appendix E, I indicate a new method of proof of (20) that is related to a remark of Lando (1998, p. 107). Such a proof reveals that $u^{\text {pre }}$ follows the same kind of dynamics followed by the bond price function of the previous subsection (for technical details, compare eq. (E1) with eq. (E3) in appendix E). Therefore, the conclusions of proposition 9 also apply to $u^{\text {pre }}(r, \tau, T)$ once that the expectation $\mathbb{E}_{\mathcal{S}}\left\{u\left(r+a_{2}(r) \mathcal{S}, \tau, T\right)\right\}$ of the previous subsection is replaced with $\bar{u}(\tau)$, and the condition $a_{2}^{\prime}=0$ stated in prop. 9 (parts b and c) is ignored.

\section{Extensions and conjectures}

This section provides comparative statics results relating bond prices to risk-premia, "volatility of volatility" of the short-term rate, and correlation between volatility and the short-term rate. With the exception of the comparative statics on risk-premia, however, some of the following results are not neat. They would be neat only after confirming a couple of reasonable conjectures that I shall be formulating in coming up with results. It is worth stressing again (see footnote 10) that results such as the ones appearing in this section (as well as the comparative statics results appearing in Bergman, Grundy and Wiener (1996, sect. 3)) should be interpreted with the usual care needed to interpret any partial equilibrium analysis.

\subsection{The effects of risk-premia}

Let $p^{(r)}(r, y, \ell) \equiv \sum_{j=1}^{3} \Lambda^{j}(r, y, \ell) \sigma^{(j)}(r, y, \ell)$ denote the short-term rate risk premium, and consider two economies $A$ and $B$ displaying two risk-premium functions $p_{j}^{(r)}(r, y, \ell)$ such that for each $(r, y, \ell) \in \mathbb{R}_{++} \times \mathbb{R} \times \mathbb{R}, p_{A}^{(r)}(r, y, \ell)>p_{B}^{(r)}(r, y, \ell)$. Let $u^{A}$ and $u^{B}$ be the price functions in the two economies. I consider the simple thought-experiment in which a "change" in $p^{(r)}$ does not imply any changes in the various functions defined under the physical measure. Accordingly, $b_{j}=\widehat{b}+p_{j}^{(r)}$ and the price difference $\nabla u=u^{A}-u^{B}$ 
satisfies

$$
\left\{\begin{array}{l}
0=\left(\frac{\partial}{\partial \tau} \nabla u+L-r\right) \nabla u+\left(p_{A}^{(r)}-p_{B}^{(r)}\right) u_{1}^{B}, \forall(r, y, \ell, \tau) \in \mathbb{R}_{++} \times \mathbb{R} \times \mathbb{R} \times[t, T) \\
\nabla u(r, y, T, T)=0, \forall(r, y, \ell) \in \mathbb{R}_{++} \times \mathbb{R} \times \mathbb{R}
\end{array}\right.
$$

where $L \nabla u=b^{A} \nabla u_{1}+\sigma \nabla u_{11}+\varphi \nabla u_{2}+\psi \nabla u_{22}+\varepsilon \nabla u_{3}+\pi \nabla u_{33}+\left(\sum_{j=1}^{3} \sigma^{(j)} \psi^{(j)}\right) \nabla u_{12}+$ $\left(\sum_{j=1}^{3} \sigma^{(j)} \pi^{(j)}\right) \nabla u_{13}+\left(\sum_{j=1}^{3} \psi^{(j)} \pi^{(j)}\right) \nabla u_{23}$. Therefore, $u^{A}<u^{B}$ whenever $u_{1}^{B}<0$. Clearly, this result extends proposition 3; in particular, it shows that the yield curve is not necessarily uniformly increasing in the short-term rate risk-premium: as illustrated in section 4 and appendix $\mathrm{C}$, there are cases in which $u_{1}>0$ at medium-long maturity dates. In these cases, an increase in $\Lambda^{1}$ might make the yield curve increase at short maturity dates and decrease at medium-long maturity dates, but no formal proof of such a claim is provided here.

The effects of an increase of the volatility (and other factors) risk-premium are similar. For each $(r, y, \ell) \in \mathbb{R}_{++} \times \mathbb{R} \times \mathbb{R}$, let $p^{(y)}(r, y, \ell) \equiv \sum_{j=1}^{3} \Lambda^{j}(r, y, \ell) \sigma^{(j)}(r, y, \ell)$, $\varphi=\widehat{\varphi}-p^{(y)}(r, y, \ell)$, and suppose that $p_{A}^{(y)}(r, y, \ell)>p_{B}^{(y)}(r, y, \ell)$. If $u^{A}$ and $u^{B}$ are the corresponding bond prices, one has that $\forall(r, y, \ell, \tau) \in \mathbb{R}_{++} \times \mathbb{R} \times \mathbb{R} \times[t, T), u^{A}(r, y, \ell, \tau, T)>$ $u^{B}(r, y, \ell, \tau, T)$ if $-\left(p_{A}^{(y)}(r, y, \ell)-p_{B}^{(y)}(r, y, \ell)\right) \cdot u_{2}^{B}(r, y, \ell, \tau, T)>0$.

\subsection{The volatility of volatility, and correlation issues}

Now consider two economies $A$ and $B$ which only differ because volatility is more volatile in economy $A$ than in economy $B$, i.e., for each $(r, y, \ell) \in \mathbb{R}_{++} \times \mathbb{R} \times \mathbb{R}, \psi^{A}(r, y, \ell)>$ $\psi^{B}\left(r, y, \ell\right.$ ) and $\sigma_{2}>0$ (a similar analysis can be performed for factor $\ell$ ). I consider the thought-experiment in which two economies are compared when only function $\psi^{(2)}$ changes (recall that $\psi \equiv \sum_{j=1}^{3}\left(\psi^{(j)}\right)^{2} / 2$ ). Clearly, function $\psi^{(2)}$ may change sign on $\mathbb{R}_{++} \times \mathbb{R} \times \mathbb{R}$ but to simplify, I assume that this is not the case. To fix ideas, I take $\psi^{(2)}>0$. In addition, I impose that the various functions defined under the physical measure are unchanged with the exception of functions $\sigma^{(2)}$ and $\pi^{(2)}$, which are the effective channels of $\psi^{(2)}$-induced correlation among factors. Let $\varepsilon \equiv \widehat{\varepsilon}-p^{(\ell)}$, where $p^{(\ell)} \equiv \sum_{j=1}^{3} \Lambda^{j} \pi^{(j)}$. Proceeding as in the previous subsection, I find that $\forall(r, y, \ell, \tau) \in \mathbb{R}_{++} \times \mathbb{R} \times \mathbb{R} \times[t, T)$, $u^{A}(r, y, \ell, \tau, T)>u^{B}(r, y, \ell, \tau, T)$ whenever

$$
\begin{aligned}
& \left(\Lambda_{A}^{2} \sigma_{A}^{2}-\Lambda_{B}^{2} \sigma_{B}^{2}\right) u_{1}^{B}+\left(\sigma^{A}-\sigma^{B}\right) u_{11}^{B}-\left(\Lambda_{A}^{2} \psi_{A}^{2}-\Lambda_{B}^{2} \psi_{B}^{2}\right) u_{2}^{B}+\left(\psi^{A}-\psi^{B}\right) u_{22}^{B} \\
& -\left(\Lambda_{A}^{2} \pi_{A}^{2}-\Lambda_{B}^{2} \pi_{B}^{2}\right) u_{3}^{B}+\left(\pi^{A}-\pi^{B}\right) u_{33}^{B}+\left(\sigma_{A}^{2} \psi_{A}^{2}-\sigma_{B}^{2} \psi_{B}^{2}\right) u_{12}^{B} \\
& +\left(\sigma_{A}^{2} \pi_{A}^{2}-\sigma_{B}^{2} \pi_{B}^{2}\right) u_{13}^{B}+\left(\psi_{A}^{2} \pi_{A}^{2}-\psi_{B}^{2} \pi_{B}^{2}\right) u_{23}^{B}>0 .
\end{aligned}
$$

By lemma D1 in appendix D, $u_{1}$ dominates $u_{11}, u_{2}, u_{22}, u_{3}, u_{33}, u_{12}, u_{13}$ and $u_{23}$ at short maturity dates. It follows that at short maturity dates, $u^{A}(r, y, \ell, \tau, T)>u^{B}(r, y, \ell, \tau, T)$ as long as $\left(\Lambda_{A}^{2} \sigma_{A}^{2}-\Lambda_{B}^{2} \sigma_{B}^{2}\right) u_{1}^{B}>0$ : at short-maturity dates, the effect of the volatility of volatility has an impact on bond prices through the channel of the short-term rate/volatility correlation. In the absence of such a correlation, $\sigma^{(2)}$ would be zero. Suppose that $\pi^{(2)}$ is also nil. Results in appendix A suggest that it is a reasonable conjecture that $u_{2}$ dominates $u_{22}$ and $u_{23}$ at short maturity dates (this conjecture is 
always true in affine models in which the initial state is free). If this is actually the case, $u^{A}(r, y, \ell, \tau, T)>u^{B}(r, y, \ell, \tau, T)$ whenever $\left(\Lambda_{A}^{2} \psi_{A}^{2}-\Lambda_{B}^{2} \psi_{B}^{2}\right) u_{2}^{B}<0$.

As regards two examples illustrating the previous results, consider the Dai and Singleton (2000) models (13) and (16). In appendix D, I show that at short-maturity dates, model (13) satisfies inequality (21) at the parameter estimates reported by the authors. In the same appendix, I show that given the parameter estimates reported by the authors, model (16) satisfies inequality (21) for any finite maturity date.

\section{Conclusion}

This paper has analyzed theoretical properties of standard, parsimonious term-structure models in which bond prices are modeled starting from the knowledge of the stochastic evolution of the short-term rate. Consistently with an approach followed by many authors over nearly thirty years, the short-term rate was assumed to be a diffusion process. The objective here was to study how arbitrage restrictions, together with additional properties, such as convexity of bond prices with respect to the short-term rate, implied restrictions on the dynamics of the short-term rate and other (possibly unobservable) factor processes. In addition to provide a theoretical construction of how rationally formed bond prices move in reaction to observable and unobservable factor changes, the theory developed in this article imposes new testable restrictions on the joint dynamics of bond prices, fundamentals and models of risk-aversion. Typical examples of such restrictions are summarized in property VIII) stated in the introduction, or in table 1 presented in section 4.1. According to property VIII), for instance, it can not be the case that the risk-neutral drift of the short-term rate is increasing in volatility and that bond prices are increasing in volatility at short maturity dates: this is a testable implication of any stochastic volatility model of the short-term rate in a diffusion setting. There is a recent increase of interest in asking data to give detailed information on the validity of generic properties of asset pricing models. Bakshi, Cao and Chen (2000), for instance, recently submitted the fundamental, "general properties of option prices"

of Bergman, Grundy and Wiener (1996) to a thorough empirical examination. Similar empirical studies can be conducted within the context analyzed in this article, especially when the scope is to deepen our understanding of the relationship between bond prices and volatility. 


\section{Appendix A: Regularity conditions and lemmata}

The main objective of this appendix is to impose regularity conditions that can be met by the typical models of the short-term rate proposed so far in the literature. While such conditions are met by virtually all the scalar diffusions examples of section 3 , the sufficient conditions I use to treat the stochastic volatility case are relatively stronger. More or less, these conditions are the same as those previously used in the stochastic volatility option pricing area, but they were rarely spelled out, as in the present appendix.

I start with presenting conditions for the difficult multidimensional case that I will occasionally use to treat the various stochastic volatility models (lemma A1, assumption A1 and lemma A2). Then I present conditions that are used to treat the scalar diffusion case of section 3 (assumption A2), and that are considerably less stringent than those required by El Karoui, Jeanblanc-Picqué and Shreve (1998) in the one-dimensional option pricing domain.

Lemma A1. Let $N$ and $M$ be integer numbers, and let $(\Omega, \mathcal{F}, \mathbb{F}, Z)$ be a probability space, where $\mathbb{F}=\{\mathcal{F}(\tau)\}_{\tau \in[t, T]}$ is the $Z$-augmentation of the natural filtration $\mathcal{F}^{W}(\tau)=$ $\sigma(W(s), s \leq \tau)$ generated by a $N$-dimensional $Z$-Brownian motion $W($ with $\mathcal{F}=\mathcal{F}(T))$. Suppose that for any fixed $T, f(\cdot, \cdot, T)$ is continuous, it is of class $C^{2,1}\left(\mathbb{R}^{N} \times[t, T]\right)$, it is solution of the following partial differential equation

$$
\left\{\begin{array}{l}
0=\left(\frac{\partial}{\partial t}+L-k\right) f(z, \tau, T)+h(z, \tau), \forall(z, \tau) \in \mathbb{R}^{N} \times[t, T) \\
f(z, T, T)=H(z), z \in \mathbb{R}^{N}
\end{array}\right.
$$

where $k(z, \tau)>0$,

$$
L f(z, \tau, T) \equiv f_{z}(z, \tau, T) b(x, \tau)+\frac{1}{2} \operatorname{tr}\left\{v(z, \tau) v^{\mathrm{T}}(z, \tau) f_{z z}(z, \tau)\right\},
$$

and $v(z, \tau): \mathbb{R}^{N} \times[t, T] \mapsto \mathbb{R}^{N \times M}$ (the space of the $N \times M$ real matrices), $b(z, \tau)$ : $\mathbb{R}^{N} \times[t, T] \mapsto \mathbb{R}^{N},(k, h)(z, \tau): \mathbb{R}^{N} \times[t, T] \mapsto\left(\mathbb{R}_{+}, \mathbb{R}\right)$, and satisfies the polynomial growth condition

$$
\max _{\tau \in[t, T]} f(z, \tau, T) \leq C_{f}\left(1+\|z\|^{2 c_{f}}\right), z \in \mathbb{R}^{N},
$$

for some constants $C_{f}>0, c_{f} \geq 1$. Then, under the conditions given in the proof,

$$
f(z(t), t, T)=\mathbb{E}\left\{\int_{t}^{T} \kappa(t, \tau) h(z(\tau), \tau) d \tau\right\}+\mathbb{E}\{\kappa(t, T) H(z(T))\},
$$

where $\kappa(t, \tau) \equiv \exp \left(-\int_{t}^{\tau} k(z(u), u) d u\right)$, and $z$ is the solution of the following stochastic differential system

$$
d z(\tau)=b(z(\tau), \tau) d \tau+v(z(\tau), \tau) d W(\tau), \tau \in[t, T] .
$$

Proof. This is the Feynman-Kac stochastic representation of the (unique) solution of a partial differential equation. Regularity conditions that are needed for such a representation to hold can be found in Karatzas and Shreve (1991, p. 366) for instance, and are: 
(H1) $\max _{\tau \in[t, T]}|h(z, \tau)| \leq C_{h}\left(1+\|z\|^{2 c_{h}}\right), z \in \mathbb{R}^{N}$, and $|H(z)| \leq C_{h}\left(1+\|z\|^{2 c_{h}}\right)$, $z \in \mathbb{R}^{N}$, for some constants $C_{h}>0, c_{h} \geq 1$.

(H2) The coefficients $v, b$ are continuous and satisfy the linear growth condition: $\forall \tau \in$ $[t, T], z \in \mathbb{R}^{N},\|b(\tau, z)\|^{2}+\|v(\tau, z)\|^{2} \leq C_{b v}\left(1+\|z\|^{2}\right)$ for some positive constant $C_{b v}$.

Naturally, the existence of the Feynman-Kac representation does not ensure per se the existence of a solution to the partial differential equation (A1). In the stochastic volatility sections of this paper, I shall be drawing on the conditions used by Krylov (1980) to guarantee it:

Assumption A1. The functions $b, v, h, H, k$ are continuous, they satisfy a Lipschitz condition, and are twice differentiable. The resulting derivatives are continuous and satisfy a growth condition. $k$ is nonnegative.

Lemma A2. If assumption A1 holds, there exists a unique solution to (A1) that satisfies the polynomial growth condition (A2) and that admits the representation (A3).

Proof. See Krylov (1980). ॥

The next assumption presents much less stringent conditions that will be used in the scalar diffusion setting.

Assumption A2. Let $N=1$ and let the domain of $z$ be $(0, \infty)$. In addition to the maintained assumptions of the main text concerning the behavior of $v, b$, the following conditions hold true:

(H3) The speed measure, defined [as in Karlin and Taylor (1981, p. 195)] as the integral of $m(r) \equiv \frac{1}{v(r)^{2}} \exp \left(-\int_{r}^{\delta} \frac{2 b(u)}{v(u)^{2}} d u\right)$, converges at both boundaries of $(0, \infty)$; and the scale measure, defined as the integral of $s(r) \equiv \exp \left(\int_{r}^{\delta} \frac{2 b(u)}{v(u)^{2}} d u\right)$, diverges at both boundaries of $(0, \infty)$.

(H4) $h$ is left limit continuous and satisfies the polynomial condition (H1) given in lemma A1 above. ${ }^{17}$

(H5) $\int_{t}^{T} \int_{0}^{\infty}|f(z, \tau, T)| \exp \left(-c \cdot z^{2}\right) d z d \tau<\infty$ for some constant $c>0$

The previous conditions were used by Aït-Sahalia $(1996 a, b)$, and as discussed at length by Aït-Sahalia (1996b, appendix 1), the maintained assumptions of the main text

\footnotetext{
${ }^{17}$ Actually, Aït-Sahalia (1996b, appendix 3) even allowed for an exponential growth condition for $h$ rather than for the more stringent polynomial condition $(\mathrm{H} 1)$.
} 
imply that $v, b$ satisfy a sort of local Lipschitz conditions, ${ }^{18}$ and are able to guarantee the existence of a unique strong solution for (A4) up possibly to an explosion time; condition (H3) then ensures that no explosions can occur in finite expected time, thus making $z$ a stationary process. Very well written accounts of the so-called Feller's tests for explosions can be found in Karlin and Taylor (1981, p. 226-242) or in Karatzas and Shreve (1991, p. 342-353). The role of (H4) and (H5), instead, is to guarantee that a unique solution of $(\mathrm{A} 1)$ exists, that is continuous and $C^{2,1}((0, \infty) \times[t, T])$, and that admits a representation in terms of the fundamental solution of the partial differential equation (A1), or the Green function. Notice that the class of functions $f$ belongs to is defined by condition (H5) and not by condition (A2) of lemma A1. As Aït-Sahalia (1996b, appendix 3) noted, a solution would fail to be unique when (H5) is replaced by a less severe boundedness condition. The representation Aït-Sahalia (1996b, lemma 1) uses to study his bond pricing problem is then in terms of the Green function. By a slight generalization of formulae (7.26)-(7.29) p. 369 in Karatzas and Shreve (1991), however, such a representation is equivalent to the Feynman-Kac representation (A3) of lemma A1, which is exactly what I need below.

I am now in a position to state the first, preliminary results that will be repeatedly used in appendices B, C and D. Assumption A3 below contains sufficient conditions that are adapted from the conditions presented above; such conditions ensure the existence of the Feynman-Kac representation for the relevant partial derivatives of the bond price in the setting of the models that I analyze here (see lemma A3). Such a representation is stated in its most general form; hence, many of the conditions listed in assumption A3 below are not always needed and in fact will often be relaxed in appendices B and C. Lemma A4 shows how the solution of stochastic differential system behaves in response to a perturbation of the initial condition: as originally noticed by Bergman, Grundy and Wiener (1996) in the option pricing domain, only in the scalar diffusion case will the "no-crossing" property mentioned in section 1 apply in great generality. Lemma A5 combines lemma A4 with the L'Hôpital's rule to show that as the maturity of the bond goes to zero, the limit of $u_{11} / u_{1}$ becomes zero.

Assumption A3. The functions

(H6) $b, b_{1}, b_{2}, b_{11}, \sigma, \sigma_{1}, \sigma_{11}$

(H7) $\sigma_{2}$

(H8) $\varphi, \varphi_{1}, \varphi_{2}, \varphi_{11}$

(H9) $\psi, \psi_{1}, \psi_{2}, \psi_{11}$

of system (1) are continuous, satisfy a Lipschitz condition, and are twice differentiable. The resulting derivatives are continuous and satisfy a growth condition. In addition,

(H10) For each $(r, y) \in(0, \infty) \times(-\infty, \infty)$, the function $r-\varphi_{2}(r, y) \in(0, \infty)$.

\footnotetext{
${ }^{18}$ In this case, the Lipschitz coefficients are allowed to vary over any compact subset of the state space, rather than being fixed on $(0, \infty)$.
} 
(H11) For each $(r, y) \in(0, \infty) \times(-\infty, \infty)$, the function $r-b_{1}(r, y) \in(0, \infty)$.

(H12) For each $(r, y) \in(0, \infty) \times(-\infty, \infty)$, the function $r-2 b_{1}(r, y)-\sigma_{11}(r, y) \in(0, \infty)$.

Conditions (H10) and (H11) are very easy to interpret. They are always satisfied, for instance, when the drift functions of the short-term rate and volatility are consistent with a kind of very strong stability property stating that the drift function of a diffusion has to be negatively sloped with respect to the solution flow, with a slope that is bounded. In practice, condition (H10) is amply satisfied by many of the scalar diffusion examples given in appendix B. Condition (H12) is stronger than (H10)-(H11), but in practice it is also amply satisfied in many of the scalar diffusion examples analyzed in this paper. Finally, all the stochastic volatility examples of section 4 satisfy condition (H10) which, in addition to the conditions in $\mathrm{C} 2$, is the only condition I actually need in order to show proposition 6 and, more generally, the existence of a solution to eq. (11) in the main text (as well as the existence of a Feynman-Kac stochastic representation of such a solution).

The reader, however, may wish to consider an alternate set of less stringent conditions that can be useful when one analyzes nonlinear models. This is:

(H13) The processes $\left(\kappa^{i}(t, \tau)\right)_{\tau \in(t, T]}, i=1,2,3$, given in eqs. (A5) of lemma A3 below are in $(0,1)$ almost surely.

Introduce now the following abstract notation: for a given function $f$ belonging to the sets (H6)-(H9) of assumption A3 above, set $f_{(i)} \equiv f\left(\vartheta_{(i)}, \phi_{(i)}\right)$. As an example, $b_{(i)} \equiv b\left(\vartheta_{(i)}, \phi_{(i)}\right)$, or $\psi_{(3), 1} \equiv \psi_{1}\left(\vartheta_{(3)}, \phi_{(3)}\right)$. The indices on $\vartheta$ and $\phi$ will be introduced during the statement of lemma A3 below.

Lemma A3 (Feynman-Kac representation of the partial derivatives of the bond price with respect to the short-term rate and volatility). Let assumption A3 hold and let $w^{1}(x, s, t, T) \equiv u_{2}(x, s, t, T), w^{2}(x, s, t, T) \equiv u_{1}(x, s, t, T), w^{3}(x, s, t, T) \equiv u_{11}(x, s, t, T)$ satisfy the polynomial condition (A2). Let, finally, $h^{i}$ in (A6) below satisfy Lipschitz and growth conditions. We have:

$$
w^{i}(x, s, t, T)=\mathbb{E}\left\{\int_{t}^{T} \kappa^{i}(t, \tau) h^{i}\left(\vartheta_{(i)}(\tau), \phi_{(i)}(\tau), \tau, T\right) d \tau\right\}, \quad i=1, \cdots, 3,
$$

where

$$
\left\{\begin{array}{l}
\kappa^{1}(t, \tau)=\exp \left\{-\int_{t}^{\tau}\left(\vartheta_{(1)}(u)-\varphi_{2}\left(\vartheta_{(1)}(u), \phi_{(1)}(u)\right)\right) d u\right\} \\
\kappa^{2}(t, \tau)=\exp \left\{-\int_{t}^{\tau}\left(\vartheta_{(2)}(u)-b_{1}\left(\vartheta_{(2)}(u), \phi_{(2)}(u)\right)\right) d u\right\} \\
\kappa^{3}(t, \tau)=\exp \left\{-\int_{t}^{\tau}\left(\vartheta_{(3)}(u)-2 b_{1}\left(\vartheta_{(3)}(u), \phi_{(3)}(u)\right)-\sigma_{11}\left(\vartheta_{(3)}(u), \phi_{(3)}(u)\right)\right) d u\right\}
\end{array}\right.
$$




$$
\left\{\begin{aligned}
h^{1}= & b_{(1), 2} w^{2}+\sigma_{(1), 2} w^{3} \\
h^{2}= & -u+\varphi_{(2), 1} w^{1}+\psi_{(2), 1} u_{22} \\
h^{3}= & \left(2-b_{(3), 11}\right) w^{2}+\varphi_{(3), 11} w^{1}+\psi_{(3), 11} u_{22} \\
& +2 \psi_{(3), 1} u_{122}+\left(2 \varphi_{(3), 1}+\frac{\partial^{2}}{\partial \vartheta^{2}}\left\{\sigma_{(3)}^{(1)} \psi_{(3)}^{(1)}+\sigma_{(3)}^{(2)} \psi_{(3)}^{(2)}\right\}\right) u_{12}
\end{aligned}\right.
$$

$\left(\vartheta_{(i)}, \phi_{(i)}\right)_{i=1}^{3}$ are solutions of the following stochastic differential systems:

$$
\begin{aligned}
& \left\{\begin{array}{l}
d \vartheta_{(1)}=\left(b_{(1)}+\frac{\partial}{\partial \phi} \sum_{j=1}^{2} \sigma_{(1)}^{(j)} \psi_{(1)}^{(j)}\right) d \tau+\sigma_{(1)}^{(1)} d W+\sigma_{(1)}^{(2)} d B \\
d \phi_{(1)}=\left(\varphi_{(1)}+\psi_{(1), 2}\right) d \tau+\psi_{(1)}^{(1)} d W+\psi_{(1)}^{(2)} d B
\end{array}\right. \\
& \left\{\begin{array}{l}
d \vartheta_{(2)}=\left(b_{(2)}+\sigma_{(2), 1}\right) d \tau+\sigma_{(2)}^{(1)} d W+\sigma_{(2)}^{(2)} d B \\
d \phi_{(2)}=\left(\varphi_{(2)}+\frac{\partial}{\partial \vartheta} \sum_{j=1}^{2} \sigma_{(2)}^{(j)} \psi_{(2)}^{(j)}\right) d \tau+\psi_{(2)}^{(1)} d W+\psi_{(2)}^{(2)} d B
\end{array}\right. \\
& \left\{\begin{array}{l}
d \vartheta_{(3)}=\left(b_{(3)}+2 \sigma_{(3), 1}\right) d \tau+\sigma_{(3)}^{(1)} d W+\sigma_{(3)}^{(2)} d B \\
d \phi_{(3)}=\left(\varphi_{(3)}+2 \frac{\partial}{\partial \vartheta} \sum_{j=1}^{2} \sigma_{(3)}^{(j)} \psi_{(3)}^{(j)}\right) d \tau+\psi_{(3)}^{(1)} d W+\psi_{(3)}^{(2)} d B
\end{array}\right.
\end{aligned}
$$

and $b_{(i), 1} \equiv \frac{\partial}{\partial \vartheta} b\left(\vartheta_{(i)}, \phi_{(i)}\right), \varphi_{(i)} \equiv \varphi\left(\vartheta_{(i)}, \phi_{(i)}\right), \psi_{(i), 2} \equiv \frac{\partial}{\partial \phi} \psi\left(\vartheta_{(i)}, \phi_{(i)}\right)$, etc.

Proof. It is easily checked that assumption A3 is as assumption A1 in lemma A2. Then, by taking the appropriate partial derivatives in eq. (3), one obtains that $w^{i}, i=1,2,3$, are solutions of the following partial differential equations

$$
\left\{\begin{array}{l}
0=\left(\frac{\partial}{\partial t}+L^{i}-k^{i}\right) w^{i}(\vartheta, \phi, \tau, T)+h^{i}(\vartheta, \phi, \tau, T), \forall(\vartheta, \phi, \tau) \in \mathbb{R}_{+} \times \mathbb{R} \times[t, T) \\
w^{i}(\vartheta, \phi, T, T)=0, \forall(\vartheta, \phi) \in \mathbb{R}_{+} \times \mathbb{R}
\end{array}\right.
$$

where $h^{i}, 1,2,3$, are as in (A6),

$$
\left\{\begin{array}{l}
k^{1}(\vartheta, \phi)=\vartheta-\varphi_{2}(\vartheta, \phi) \\
k^{2}(\vartheta, \phi)=\vartheta-b_{1}(\vartheta, \phi) \\
k^{3}(\vartheta, \phi)=\vartheta-2 b_{1}(\vartheta, \phi)-\sigma_{11}(\vartheta, \phi)
\end{array}\right.
$$


and

$$
\left\{\begin{array}{l}
L^{1} w^{1}=\left(b+\frac{\partial}{\partial \phi} \sum_{j=1}^{2} \sigma^{(j)} \psi^{(j)}\right) w_{1}^{1}+\left(\varphi+\psi_{2}\right) w_{2}^{1}+\sigma w_{11}^{1}+\psi w_{22}^{1}+\left(\sum_{j=1}^{2} \sigma^{(j)} \psi^{(j)}\right) w_{12}^{1} \\
L^{2} w^{2}=\left(b+\sigma_{1}\right) w_{1}^{2}+\left(\varphi+\frac{\partial}{\partial \vartheta} \sum_{j=1}^{2} \sigma^{(j)} \psi^{(j)}\right) w_{2}^{2}+\sigma w_{11}^{2}+\psi w_{22}^{2}+\left(\sum_{j=1}^{2} \sigma^{(j)} \psi^{(j)}\right) w_{12}^{2} \\
L^{3} w^{3}=\left(b+2 \sigma_{1}\right) w_{1}^{3}+\left(\varphi+2 \frac{\partial}{\partial \vartheta} \sum_{j=1}^{2} \sigma^{(j)} \psi^{(j)}\right) w_{2}^{3}+\sigma w_{11}^{3}+\psi w_{22}^{3}+\left(\sum_{j=1}^{2} \sigma^{(j)} \psi^{(j)}\right) w_{12}^{3}
\end{array}\right.
$$

The result then follows by lemma A2. ॥

Remark A1. If $N=1$ and $\sigma \equiv 1$, the previous result concerning the stochastic representation of $w^{2}$ and $w^{3}$ holds by only assuming that in addition to (H11)-(H12), the coefficients in eq. (1) and (A7) and functions $h^{2}$ and $h^{3}$ satisfy the conditions of assumption A2, with $w^{2}$ and $w^{3}$ satisfying (H5).

Remark A2. An alternative set of sufficient conditions that can be used in the stochastic volatility setting (see assumptions $\mathrm{C} 1$ and $\mathrm{C} 2$ in appendix $\mathrm{C}$ ) imposes uniform ellipticity, boundedness and Hölder continuity on the coefficients of (A8); and polynomial growth on $h^{i}$ (see, for instance, Karatzas and Shreve (1991, remark 7.8 p. 368)).

Remark A3. The previous conditions are sufficient conditions for existence of a unique solution to Cauchy problems. When such a solution is already known to exist (as for instance in the Longstaff and Schwartz (1992) model presented in the main text), the less stringent conditions in lemma A1 can be applied to ensure the Feynman-Kac representation of the partial derivatives of the bond price. In addition, the conditions in remark A2 can be relaxed considerably by making opportune localizations (see Friedman (1975)).

The following lemma formalizes the idea that at small $\Delta t$, the short-term rate $r(t+\Delta t)$ is approximately equal to $x$.

Lemma A4 (Sensitivity of the short-term rate with respect to the initial condition). Let $(\vartheta, \phi)(\tau) \equiv(\vartheta, \phi)(x, s, \tau ; \omega)(\tau \in[t, T])$ denote the flow of the stochastic differential equations (1) at $\tau$ starting at $(x, s)$ in $t$ at the point $\omega \in \Omega$. If $\sigma_{2} \equiv 0$, let the coefficients of equations (1) satisfy assumption A2; otherwise, let such coefficients satisfy standard Lipschitz and growth conditions. We have:

(a) $\lim _{\tau \downarrow t} \frac{\partial \vartheta}{\partial x}(\tau)=1$; and,

(b) $\lim _{\tau \downarrow t} \frac{\partial^{2} \vartheta}{\partial x^{2}}(\tau)=0$.

Proof. By theorems 4.7.1 and 4.7.2 p. 177 in Kunita (1990), there exists (up to an explosion time) a unique forward stochastic flow of local $C^{2}$-diffeomorphisms $(\vartheta, \phi)$ in 
$(x, s)$. The processes $\partial \vartheta / \partial x$ and $\partial \phi / \partial x$ then satisfy:

$$
\left\{\begin{array}{r}
\frac{\partial \vartheta}{\partial x}(\tau)=1+\int_{t}^{\tau}\left(b_{1} \frac{\partial \vartheta}{\partial x}+b_{2} \frac{\partial \phi}{\partial x}\right)(u) d u+\int_{t}^{\tau}\left\{\left(\sigma_{1}^{(1)} \frac{\partial \vartheta}{\partial x}+\sigma_{2}^{(1)} \frac{\partial \phi}{\partial x}\right)(u) d W(u)\right. \\
\left.+\left(\sigma_{1}^{(2)} \frac{\partial \vartheta}{\partial x}+\sigma_{2}^{(2)} \frac{\partial \phi}{\partial x}\right)(u) d B(u)\right\} \\
\frac{\partial \phi}{\partial x}(\tau)=\int_{t}^{\tau}\left(\varphi_{1} \frac{\partial \vartheta}{\partial x}+\varphi_{2} \frac{\partial \phi}{\partial x}\right)(u) d u+\int_{t}^{\tau}\left\{\left(\psi_{1}^{(1)} \frac{\partial \vartheta}{\partial x}+\psi_{2}^{(1)} \frac{\partial \phi}{\partial x}\right)(u) d W(u)\right. \\
\left.+\left(\psi_{1}^{(2)} \frac{\partial \vartheta}{\partial x}+\psi_{2}^{(2)} \frac{\partial \phi}{\partial x}\right)(u) d B(u)\right\}
\end{array}\right.
$$

from which (a)-(b) follow. ॥

Lemma A5 (Slope effects dominate convexity effects at short maturity dates). Let the coefficients of $L$ in eq. (3) satisfy assumption A2 if $\sigma_{2} \equiv 0$; otherwise, let such coefficients satisfy assumption $\mathrm{A} 1$. Then, for each $(x, s) \in \mathbb{R}_{+} \times \mathbb{R}, \lim _{T \downarrow t} \frac{u_{11}}{u_{1}}(x, s, t, T)=$ 0.

Proof. By lemma A2 and the discussion after assumption A2, the assumptions of this lemma ensure the existence of the Keynman-Kac stochastic representation in eq. (4) of the main text. Clearly, $\lim _{T \downarrow t} u_{11}(x, s, t, T)$ and $\lim _{T \downarrow t} u_{1}(x, s, t, T)$ are both zero (use, for instance, the Lebesgue's dominated convergence theorem in (5) and (6) as I do in showing proposition 2 in appendix B; alternatively, just notice that $\lim _{T \downarrow t} u_{11}(x, s, t, T)$ and $\lim _{T \downarrow t} u_{1}(x, s, t, T)$ both equal zero by the boundary conditions in eqs. (A8) given in the course of the proof of lemma A3). However, $\lim u_{11} / u_{1}$ can be written as:

$$
\begin{aligned}
& \lim _{T \downarrow t} \frac{u_{11}}{-u_{1}}(x, s, t, T) \\
= & \lim _{T \downarrow t} \frac{\mathbb{E}\left\{\left[\left(\int_{t}^{T} \frac{\partial \vartheta}{\partial x}(\tau) d \tau\right)^{2}-\int_{t}^{T} \frac{\partial^{2} \vartheta}{\partial x^{2}}(\tau) d \tau\right] \cdot \exp \left(-\int_{t}^{T} \vartheta(\tau) d \tau\right)\right\}}{\mathbb{E}\left\{\left(\int_{t}^{T} \frac{\partial \vartheta}{\partial x}(\tau) d \tau\right) \cdot \exp \left(-\int_{t}^{T} \vartheta(\tau) d \tau\right)\right\}} \\
= & \lim _{T \downarrow t} \frac{\mathbb{E}\left\{\left[(T-t)\left(\frac{1}{T-t} \int_{t}^{T} \frac{\partial \vartheta}{\partial x}(\tau) d \tau\right)^{2}-\frac{1}{T-t} \int_{t}^{T} \frac{\partial^{2} \vartheta}{\partial x^{2}}(\tau) d \tau\right] \cdot \exp \left(-\int_{t}^{T} \vartheta(\tau) d \tau\right)\right\}}{\mathbb{E}\left\{\left(\frac{1}{T-t} \int_{t}^{T} \frac{\partial \vartheta}{\partial x}(\tau) d \tau\right) \cdot \exp \left(-\int_{t}^{T} \vartheta(\tau) d \tau\right)\right\}},
\end{aligned}
$$

and the result follows from the Lebesgue's dominated convergence theorem, and the fact that since $\int \frac{\partial \vartheta}{\partial x}$ and $\int \frac{\partial^{2} \vartheta}{\partial x^{2}}$ are Riemann integrals, then $\frac{1}{T-t} \int_{t}^{T} \frac{\partial \vartheta}{\partial x}(\tau) d \tau \rightarrow \frac{\partial \vartheta}{\partial x}(t)$ and $\frac{1}{T-t} \int_{t}^{T} \frac{\partial^{2} \vartheta}{\partial x^{2}}(\tau) d \tau \rightarrow \frac{\partial^{2} \vartheta}{\partial x^{2}}(t)$ as $T \downarrow t$, where $\frac{\partial \vartheta}{\partial x}(t)=1$ by lemma A4 part (a) and $\frac{\partial^{2} \vartheta}{\partial x^{2}}(t)=0$ by lemma A4 part (b). $\|$

\section{Appendix B: proofs for section 3, and a counterexample}

Assumption B1. The coefficients in (1) and $h^{2}$ and $h^{3}$ in lemma A3 satisfy the conditions of remark $\mathrm{A} 1$ in appendix $\mathrm{A}$. 
Proof of propositions 1 and 2. In the constant volatility case, the stochastic representations of $w^{2}$ and $w^{3}$ given in lemma A1 simplify to

$$
\left\{\begin{array}{l}
w^{2}(x, t, T)=\mathbb{E}\left\{\int_{t}^{T} \kappa^{2}(t, \tau) h^{2}\left(\vartheta_{(2)}(\tau), \tau, T\right) d \tau\right\} \\
w^{3}(x, t, T)=\mathbb{E}\left\{\int_{t}^{T} \kappa^{3}(t, \tau) h^{3}\left(\vartheta_{(3)}(\tau), \tau, T\right) d \tau\right\}
\end{array}\right.
$$

where

$$
\left\{\begin{array}{l}
\kappa^{2}(t, \tau)=\exp \left\{-\int_{t}^{\tau}\left(\vartheta_{(2)}(u)-b^{\prime}\left(\vartheta_{(2)}(u)\right)\right) d u\right\} \\
\kappa^{3}(t, \tau)=\exp \left\{-\int_{t}^{\tau}\left(\vartheta_{(3)}(u)-2 b^{\prime}\left(\vartheta_{(3)}(u)\right)-a^{\prime \prime}\left(\vartheta_{(3)}(u)\right)\right) d u\right\}
\end{array}\right.
$$

and

$$
\left\{\begin{array}{l}
h^{2}=-u \\
h^{3}=-\left(2-b^{\prime \prime}\right) w^{2}
\end{array}\right.
$$

and $\vartheta_{(2)}, \vartheta_{(3)}$ denote the stochastic flows of the following equations:

$$
d r(\tau)=\left(b(r(\tau))+a^{\prime}(r(\tau))\right) d \tau+\sqrt{2 a(r(\tau))} d W(\tau)
$$

and

$$
d r(\tau)=\left(b(r(\tau))+2 a^{\prime}(r(\tau))\right) d \tau+\sqrt{2 a(r(\tau))} d W(\tau) .
$$

Since the price of a bond cannot be negative by eq. (4), the first line in (B1) and the first line in (B2) ensure that $w^{2}$ is strictly negative.

The second claim of proposition 1 is confirmed by the second line in (B2), since $w^{3}$ can be written as:

$$
w^{3}(x, t, T)=\mathbb{E}\left\{\int_{t}^{T} \kappa^{3}(t, \tau)\left(2-b^{\prime \prime}\left(\vartheta_{(3)}(\tau)\right)\right)\left(-w^{2}\left(\vartheta_{(3)}(\tau), \tau, T\right)\right) d \tau\right\} .
$$

As regards proposition 2, write the previous relation as:

$$
w^{3}(x, t, T)=\left\{\int_{t}^{T} G_{2}(x, t, \tau, T) d \tau\right\} \cdot\left\{2-\frac{\int_{t}^{T} G_{1}(x, t, \tau, T) d \tau}{\int_{t}^{T} G_{2}(x, t, \tau, T) d \tau}\right\}
$$

where

$$
\left\{\begin{array}{l}
G_{1}(x, t, \tau, T) \equiv-\mathbb{E}\left\{b^{\prime \prime}\left(\vartheta_{(3)}(\tau)\right) \cdot \kappa^{3}(t, \tau) \cdot w^{2}\left(\vartheta_{(3)}(\tau), \tau, T\right)\right\} \\
G_{2}(x, t, \tau, T) \equiv-\mathbb{E}\left\{\kappa^{3}(t, \tau) \cdot w^{2}\left(\vartheta_{(3)}(\tau), \tau, T\right)\right\}
\end{array}\right.
$$

Writing $w^{3}$ as before is justified because there exists a maturity date within which $\int_{t}^{T} G_{2} \neq 0$.

We have:

$$
w^{3}(x, t, T)>0(\text { resp. }<0) \quad \text { if } \zeta(x, t, T) \equiv \frac{\int_{t}^{T} G_{1}(x, t, \tau, T) d \tau}{\int_{t}^{T} G_{2}(x, t, \tau, T) d \tau}<2(\text { resp. }>2),
$$


and

$$
\begin{aligned}
& \lim _{T \downarrow t} \zeta(x, t, T) \\
= & \lim _{T \downarrow t} \frac{\int_{t}^{T} \mathbb{E}\left\{\kappa^{3}(t, \tau) \cdot b^{\prime \prime}\left(\vartheta_{(3)}(\tau)\right) \cdot \mathbb{E}\left\{\left(\int_{\tau}^{T} \frac{\partial \vartheta}{\partial x}(u) d u\right) \cdot \exp \left(-\int_{\tau}^{T} \vartheta(u) d u\right) / \mathcal{F}(\tau)\right\}\right\} d \tau}{\int_{t}^{T} \mathbb{E}\left\{\kappa^{3}(t, \tau) \cdot \mathbb{E}\left\{\left(\int_{\tau}^{T} \frac{\partial \vartheta}{\partial x}(u) d u\right) \cdot \exp \left(-\int_{\tau}^{T} \vartheta(u) d u\right) / \mathcal{F}(\tau)\right\}\right\} d \tau} \\
= & \lim _{T \downarrow t} \frac{\frac{1}{T-t} \int_{t}^{T} \mathbb{E}\left\{\kappa^{3}(t, \tau) \cdot b^{\prime \prime}\left(\vartheta_{(3)}(\tau)\right) \cdot \frac{\partial}{\partial T} \mathbb{E}\left\{\left(\int_{\tau}^{T} \frac{\partial \vartheta}{\partial x}(u) d u\right) \cdot \exp \left(-\int_{\tau}^{T} \vartheta(u) d u\right) / \mathcal{F}(\tau)\right\}\right\} d \tau}{\frac{1}{T-t} \int_{t}^{T} \mathbb{E}\left\{\kappa^{3}(t, \tau) \cdot \frac{\partial}{\partial T} \mathbb{E}\left\{\left(\int_{\tau}^{T} \frac{\partial \vartheta}{\partial x}(u) d u\right) \cdot \exp \left(-\int_{\tau}^{T} \vartheta(u) d u\right) / \mathcal{F}(\tau)\right\}\right\}} \\
= & b^{\prime \prime}(x) \quad \text { for each } x:\left|b^{\prime \prime}(x)\right|<\infty,
\end{aligned}
$$

by (5); the L'Hôpital's rule; the Lebesgue's dominated convergence theorem; the Liebnitz's rule (noting also that the integrands in the numerator and denominator of $\zeta$ evaluated at $T$ are both zero); continuity and differentiability of $w^{2}$ w.r.t. $T$ (ensured by the Riemann representation of $w^{2}$ in (5) and the fact that $\vartheta$ and $\partial \vartheta / \partial x$ have continuous sample paths); the fact that

$$
\lim _{T \downarrow t} \frac{\partial}{\partial T}\left\{\left(\int_{t}^{T} \frac{\partial \vartheta}{\partial x}(u) d u\right) \cdot \exp \left(-\int_{t}^{T} \vartheta(u) d u\right)\right\}=\frac{\partial \vartheta}{\partial x}(t)=1,
$$

by lemma A4; and finally because $\lim _{T \downarrow t} \kappa^{3}(t, T)=1$, by lemma A3. This shows that there exists a $\widehat{T}$ depending on $x$ such that $\forall \tau \in[t, \widehat{T}]$,

$$
\zeta(x, t, \widehat{T}(x))<2(\text { resp. }>2) \text { for each } x: b^{\prime \prime}(x)<2(\text { resp. }>2),
$$

and the result follows from (B3). ॥

A counter-example to proposition 1: the double square-root model. According to the original formulation of Longstaff (1989), the short-term rate is solution of:

$$
d r(\tau)=\left(\frac{\eta^{2}}{4}-\beta \sqrt{r(\tau)}+\lambda r(\tau)\right) d \tau+\eta \sqrt{r(\tau)} d W(\tau), \beta, \lambda, \eta>0 .
$$

Thusly formulated, model (B5) implies failure of the divergence of the "scale measure" at both boundaries (zero and infinite) (see Karlin and Taylor (1981, chap. 15) and assumption A2-(H3) in appendix A. The infinite boundary can be attained in finite expected time because the term $\lambda r$ dominates the term $\beta \sqrt{r}$ as $r \rightarrow \infty$. To avoid this, one may assume that $\lambda<0$, but this creates a negative term premium whenever $u_{1}<0$. This is the technical reason for which only the case $\lambda=0$ was considered in section 3.1. This kind of phenomena also illustrates very simply a general principle that should be 
followed to ensure stationarity in the kind of models that is considered in the present paper: at arbitrarily large values of the solution flow under the risk-neutral measure, the risk premium function should never be of a higher order than the physical drift function if one wishes to ensure positive term premia.

Finally, the drift function considered in the main text was chosen because the origin is regular (attainable) in (B5) even with $\lambda=0$ and in this case,

$$
k^{2}=\vartheta_{(2)}+\beta /\left(2 \sqrt{\vartheta_{(2)}}\right)
$$

in the representation (B1) for $w^{2}$. It can be shown that the origin is regular for the auxiliary process $\vartheta_{(2)}$ too, which makes $k^{2}$ explode in finite expected time. Therefore, no Feynman-Kac stochastic representation for $w^{2}$ is possible for model (B5). In fact, Longstaff (p. 203) shows that $w^{2}>0$ for small values of $r(t)=x$. However, this is not concluding evidence of the violation of the no-crossing property, since no Feynman-Kac stochastic representation for $u$ necessarily exists. In contrast, if the short-term rate is a double square-root process of the form indicated in the main text, both boundaries can not be attained in finite expected time, and the result that $w^{2}<0$ is restored.

Conditions for bond prices and volatility to be negatively related at any maturity date: The CIR example. In the CIR model, $\widehat{b}(r)=\kappa(\iota-r), \sqrt{2 a(r)}=\eta \sqrt{r}$ and $\Lambda(r)=\lambda \sqrt{r}$ where, using the notation of footnote $11, \iota \equiv b v^{2} / \kappa, \eta \equiv k v$, and $\lambda \equiv \epsilon / v$. I am going to provide conditions ensuring that bond prices are always decreasing in the parameter $\eta$ whenever changes in $\eta$ are "pure volatility changes", i.e. caused by changes in $k$ (see footnote 11). Such conditions are most probably much severe than required, but illustrate the mechanics of the general conditions and proof provided below.

In the CIR model, the last term in eq. (8) simplifies to: $r\left(\eta_{A}-\eta_{B}\right)\left(-u_{1}^{B}\right) \times$ $\left\{\left(\eta_{A}+\eta_{B}\right)\left(u_{11}^{B} /\left(-u_{1}^{B}\right)\right) / 2-\lambda\right\}$. Therefore, an application of the maximum principle reveals that $\nabla u \leq 0$ whenever

$$
\begin{aligned}
\lambda & >\frac{\left(\eta_{A}+\eta_{B}\right) u_{11}^{B}(\vartheta(\tau), \tau, T)}{-2 u_{1}^{B}(\vartheta(\tau), \tau, T)}=\frac{\left(\eta_{A}+\eta_{B}\right)\left(1-e^{-\gamma_{B}(T-\tau)}\right)}{\left(\kappa+\eta_{B} \lambda+\gamma_{B}\right)\left(1-e^{-\gamma_{B}(T-\tau)}\right)+2 \gamma_{B} e^{-\gamma_{B}(T-\tau)}} \\
& \equiv \Phi(\tau, T ; \lambda), \text { all } \tau \in[t, T],
\end{aligned}
$$

where $\gamma \equiv \sqrt{(\kappa+\eta \lambda)^{2}+2 \eta^{2}}$. Consider the fixed points of function $\Phi$, i.e. $\lambda_{i}(\tau, T)=$ $\Phi\left(\tau, T ; \lambda_{i}(\tau, T)\right), i=1, \cdots, n_{\tau}$, where $n_{\tau}$ is the number of fixed points of $\Phi(\tau, T ; \lambda)$, $\tau \in[t, T]$. Then $\nabla u(x, t, T)<0$ for all $\lambda>\lambda(t, T) \equiv \sup _{\tau \in[t, T]} \sup _{i \in\left\{1, \cdots, n_{T}\right\}} \lambda_{i}(\tau, T)$. Next, consider an arbitrary maturity date $T^{*}<\infty$. Then $\nabla u\left(x, t, T^{*}\right)<0$ for all $T \in\left[t, T^{*}\right]$ whenever $\lambda>\sup _{T \in\left[t, T^{*}\right]} \lambda(t, T)$.

Conditions for bond prices and volatility to be negatively related at any maturity date: Theory. Here I generalize the previous example and conditions to any diffusion model. Consider perturbing the risk premium function by defining a new risk-premium as $\bar{\Lambda}(r)=$ $\lambda^{0} \cdot \Lambda(r)$, where $\lambda^{0}$ is a positive constant. The objective is to provide conditions under 
which bond prices are decreasing in volatility at any maturity date in correspondence of a sufficiently high level of $\lambda^{0}$. By results similar to lemma A1, the solution of (8) is:

$$
\begin{aligned}
\nabla u(r, \tau, T)= & \mathbb{E}\left\{\int_{t}^{T} \kappa(t, \tau)\left(a^{A}(\vartheta(\tau))-a^{B}(\vartheta(\tau))\right) u_{11}^{B}\left(\vartheta(\tau), \tau, T ; \lambda^{0}\right) d \tau\right\} \\
& -\lambda^{0} \cdot \mathbb{E}\left\{\int_{t}^{T} \kappa(t, \tau) \cdot \zeta(\vartheta(\tau)) \cdot\left(-u_{1}^{B}\left(\vartheta(\tau), \tau, T ; \lambda^{0}\right)\right) d \tau\right\},
\end{aligned}
$$

where I have emphasized the dependence of the bond pricing function on the parameter $\lambda^{0}, \kappa(t, \tau) \equiv \exp \left(-\int_{t}^{\tau} \vartheta(u) d u\right)$ and $\zeta(\vartheta) \equiv \sqrt{2 a^{A}(\vartheta)}\left(\Lambda_{A}^{1}(\vartheta)-\Lambda_{B}^{1}(\vartheta)\right)+\left(\sqrt{2 a^{A}(\vartheta)}-\right.$ $\left.\sqrt{2 a^{B}(\vartheta)}\right) \Lambda_{B}^{1}(\vartheta)$, which is strictly positive by assumption (see the main text). The $u_{11}<0$ case is trivial, so I shall only present the $u_{11}>0$ case.

Assume that function

$$
\Phi\left(x, t, T ; \lambda^{0}\right) \equiv \frac{\mathbb{E}\left\{\int_{t}^{T} \kappa(t, \tau)\left(a^{A}(\vartheta(\tau))-a^{B}(\vartheta(\tau))\right) u_{11}^{B}\left(\vartheta(\tau), \tau, T ; \lambda^{0}\right) d \tau\right\}}{\mathbb{E}\left\{\int_{t}^{T} \kappa(t, \tau) \cdot \zeta(\vartheta(\tau)) \cdot\left(-u_{1}^{B}\left(\vartheta(\tau), \tau, T ; \lambda^{0}\right)\right) d \tau\right\}}
$$

has a finite number $n_{T}$ of fixed points:

$$
\lambda_{i}^{0}(x, t, T)=\Phi\left(x, t, T ; \lambda_{i}^{0}(x, t, T)\right), \quad i=1, \cdots, n_{T} .
$$

Then, $\nabla u(x, t, T)<0$ for any $\lambda^{0}>\sup _{i \in\left\{1, \cdots, n_{T}\right\}} \lambda_{i}^{0}(x, t, T)$. Furthermore, pick an arbitrary maturity date $T^{*}<\infty$. Then we have that $\nabla u(x, t, T)<0$ for all $T \in\left[t, T^{*}\right]$ whenever $\lambda^{0}>\sup _{t<T<T^{*}} \sup _{i \in\left\{1, \cdots, n_{T}\right\}} \lambda_{i}^{0}(x, t, T)$.

\section{Appendix C: proofs and examples for section 4}

Assumption C1. b, $a, \sigma_{1}, \sigma, \varphi, \varphi_{1}, \psi$ and $\psi_{1}$ satisfy the conditions in assumption A3 of appendix $\mathrm{A}$; in addition, (H11)-(H12) in assumption $\mathrm{A} 3$ hold, and $h^{2}, h^{3}$ in lemma A3 satisfy Lipschitz and growth conditions. Alternatively, the conditions of remark A2 hold (see also remark A3).

Assumption C2. $b, a, \sigma, \sigma_{1}, \varphi, \varphi_{2}, \psi$ and $\psi_{2}$ satisfy the conditions in assumption A3 of appendix A; in addition, (H10) in assumption A3 holds, and $h^{1}$ in lemma A3 satisfies Lipschitz and growth conditions. Alternatively, the conditions of remark A2 hold (see also remark A3).

Proof of proposition 4. Follows from lemma A3 in appendix A and a straightforward generalization of propositions 1 and 2. Precisely, the first part of proposition 1 is also valid in the stochastic volatility case because of lemma A3 (see the second relation in (A6)) and the restrictions stated in proposition 4. The other claims of proposition 4 can be shown by using the same arguments as in propositions 1 and 2 whenever there exists a maturity date $T_{*}$ such that $w^{2}<0$ for all $T \leq T_{*}$ (which indeed is ensured by 
proposition 5 shown below); by lemma A3; and finally by a strategy of proof similar to the previous one and revealing that the various integrals involving $u_{2}, u_{22}, u_{122}, u_{12}$ are dominated by the integral involving $u_{1}$ (as in lemma D1 below). $\|$

Proof of proposition 5. By lemma A3,

$$
w^{2}(x, s, t, T)=\int_{t}^{T}\left\{J_{2}(x, s, t, \tau, T)+J_{3}(x, s, t, \tau, T)-J_{1}(x, s, t, \tau, T)\right\} d \tau,
$$

where:

$$
\left\{\begin{array}{l}
J_{1}(x, s, t, \tau, T) \equiv \mathbb{E}\left\{\kappa^{2}(t, \tau) \cdot u\left(\vartheta_{(2)}(\tau), \phi_{(2)}(\tau), \tau, T\right)\right\} \\
J_{2}(x, s, t, \tau, T) \equiv \mathbb{E}\left\{\kappa^{2}(t, \tau) \cdot \varphi_{1}\left(\vartheta_{(2)}(\tau), \phi_{(2)}(\tau)\right) \cdot u_{2}\left(\vartheta_{(2)}(\tau), \phi_{(2)}(\tau), \tau, T\right)\right\} \\
J_{3}(x, s, t, \tau, T) \equiv \mathbb{E}\left\{\kappa^{2}(t, \tau) \cdot \psi_{1}\left(\vartheta_{(2)}(\tau), \phi_{(2)}(\tau)\right) \cdot u_{22}\left(\vartheta_{(2)}(\tau), \phi_{(2)}(\tau), \tau, T\right)\right\}
\end{array}\right.
$$

Therefore, $w^{2}(x, s, t, T)<0$ whenever

$$
\xi(x, s, t, T) \equiv \frac{\int_{t}^{T} J_{2}(x, s, t, \tau, T) d \tau}{\int_{t}^{T} J_{1}(x, s, t, \tau, T) d \tau}+\frac{\int_{t}^{T} J_{3}(x, s, t, \tau, T) d \tau}{\int_{t}^{T} J_{1}(x, s, t, \tau, T) d \tau}<1,
$$

and since $J_{i}, i=1,2,3$, are all continuous with respect to $\tau$ and $T$, and

$$
\lim _{T \downarrow t} \frac{\frac{1}{T-t} \int_{t}^{T} J_{i}(x, s, t, \tau, T) d \tau}{\frac{1}{T-t} \int_{t}^{T} J_{1}(x, s, t, \tau, T) d \tau}=0, \quad i=2,3,
$$

then there exists a $T_{*}$ depending on $x, s$ such that $\forall T \leq T_{*}(x, s), \xi(x, s, t, T)<1$. $\|$

Uniform bounds for $T_{*}$. Are there situations in which there exist values of $T_{*}$ in proposition 5 that are independent on the initial state $(x, s)$ ? The answer is definitely positive in the case of affine models, i.e. when the bond price functions are of the form $u(x, s, t, T)=\exp (B(T-t)+C(T-t) \cdot x+D(T-t) \cdot s)$ where $B(\cdot), C(\cdot)$ and $D(\cdot)$ are not functions of the initial state $(x, s)$. A rigorous proof is as follows. In affine models, functions $\varphi_{1}(\cdot, \cdot)$ and $\psi_{1}(\cdot, \cdot)$ reduce to two constants that with a slight abuse of notation I shall refer to as $\varphi_{1}$ and $\psi_{1}$. Eq. (C1) can then be written as:

$$
w^{2}(x, s, t, T)=\int_{t}^{T} J_{1}(t, \tau, T)\left\{\varphi_{1} \cdot D(T-\tau)+\psi_{1} \cdot D(T-\tau)^{2}-1\right\} d \tau,
$$

and since $J_{1}$ is always positive, $w^{2}<0$ for all maturity dates $T_{* *}: \varphi_{1} \cdot D\left(T_{* *}-\tau\right)+\psi_{1}$. $D\left(T_{* *}-\tau\right)^{2}<1$, all $\tau \in\left[t, T_{* *}\right]$, independently of $x, s$. Naturally, the existence of such maturity dates is guaranteed by the fact that for fixed $t, \lim _{T \rightarrow t} D(T-t)=0$ (boundary condition for $u$ ). $\|$

Conditions and examples for bond prices to be increasing in the short-term rate at medium-long maturity dates. Here the starting point is eq. (C1), which clarifies why 
cases (b) and (d) in table 1, for instance, are necessary conditions for $\partial u / \partial r>0$ at medium-long maturity dates when $\partial \psi / \partial r \leq 0$. In the general case, one has that $\partial u / \partial r>0$ at medium-long maturity dates whenever $\int h^{2}>0$ in lemma A1.

This kind of conditions can be illustrated within the LS model (9), which has a known closed-form solution that is "typical" of all models examined in section 4 (see below). As an example, LS noticed that in their model, $\partial u / \partial r$ "... is always negative for small $T-\tau$, but can become positive for bonds with longer maturities" (p. 1267). This perfectly illustrates proposition 5 . LS also point out $\partial u / \partial y$ can be of either sign, or can be positive within certain maturity dates, negative at the remaining maturity dates, and vice versa. Let us see how the theory in section 4 may help to clarify such phenomena.

According to the notation introduced by Longstaff and Schwartz (1992, p. 1264 and eq. (9), p. 1263), system (9) describes the dynamics of $r, y$ under the risk-neutral measure with coefficients: $b_{0}=\alpha \gamma+\beta \eta, b_{1}=(\beta \delta-\alpha \nu) /(\beta-\alpha), b_{2}=(\delta-\nu) /(\beta-\alpha)$, $\varphi_{0}=\alpha^{2} \gamma+\beta^{2} \eta, \varphi_{1}=\alpha \beta(\nu-\delta) /(\beta-\alpha), \varphi_{2}=(\beta \nu-\alpha \delta) /(\beta-\alpha)$, where $\delta, \nu, \gamma, \eta$ are constants entering the primitive dynamical system of the model. Therefore, it is not hard to find that the equilibrium price satisfies:

$$
\left\{\begin{array}{l}
0=\left(\frac{\partial}{\partial \tau}+L-r\right) u, \forall(r, y, \tau) \in \mathbb{D}_{\alpha, \beta} \times[t, T) \\
u(r, y, T, T)=1, \forall(r, y) \in \mathbb{D}_{\alpha, \beta}
\end{array}\right.
$$

where, for given constants $\psi_{j}$ and $\rho_{j}, j=1,2$, given below,

$$
\begin{aligned}
L u(r, y, \tau, T)= & \left(b_{0}-b_{1} r+b_{2} y\right) u_{1}(r, y, \tau, T)+\left(\varphi_{0}+\varphi_{1} r-\varphi_{2} y\right) u_{2}(r, y, \tau, T) \\
& +\frac{y}{2} \cdot u_{11}(r, y, \tau, T)+\left(\psi_{1} r+\psi_{2} y\right) u_{22}(r, y, \tau, T)+\left(\rho_{3} r+\rho_{4} y\right) u_{12}(r, y, \tau, T),
\end{aligned}
$$

and $\mathbb{D}_{\alpha, \beta}$ is the strictly positive cone defined as

$$
\mathbb{D}_{\alpha, \beta}=\left\{(r, y) \in \mathbb{R}_{++} \times \mathbb{R}_{++}: \frac{y}{r} \in(\alpha, \beta)\right\} .
$$

Under mild parameter restrictions such as those given in LS (footnote 9 p. 1264), the process $(y(\tau) / r(\tau))_{\tau \geq 0}$ can not attain the boundary $\partial \mathbb{D}_{\alpha, \beta}$ in finite expected time, and so no further transversality and/or boundary condition is needed.

The solution of (C2) reported by the authors (eq. (20) p. 1266) (with coefficients $\psi_{i}$ and $\rho_{i}$ given by $\left.\psi_{1}=-\alpha \beta(\beta+\alpha) / 2, \psi_{2}=\left(\beta^{3}-\alpha^{3}\right) /(2(\beta-\alpha)), \rho_{3}=-\alpha \beta, \rho_{4}=\beta+\alpha\right)$ is:

$$
u(x, s, t, T)=A(T-t)^{2 \gamma} \cdot B(T-t)^{2 \eta} \cdot \exp (\kappa \cdot(T-t)+C(T-t) \cdot x+D(T-t) \cdot s),
$$


where

$$
\left\{\begin{array}{l}
A(\tau)=2 \phi /((\delta+\phi)(\exp (\phi \tau)-1)+2 \phi) \\
B(\tau)=2 \bar{\psi} /((\nu+\bar{\psi})(\exp (\bar{\psi} \tau)-1)+2 \bar{\psi}) \\
C(\tau)=(\alpha \phi(\exp (\bar{\psi} \tau)-1) B(\tau)-\beta \bar{\psi}(\exp (\phi \tau)-1) A(\tau)) /(\phi \bar{\psi}(\beta-\alpha)) \\
D(\tau)=(\bar{\psi}(\exp (\phi \tau)-1) A(\tau)-\phi(\exp (\bar{\psi} \tau)-1) B(\tau)) /(\phi \bar{\psi}(\beta-\alpha))
\end{array}\right.
$$

and $\kappa=\gamma(\delta+\phi)+\eta(\nu+\bar{\psi}), \phi=\sqrt{2 \alpha+\delta^{2}}, \bar{\psi}=\sqrt{2 \beta+\nu^{2}}$.

Functions $C$ and $D$ are factor loadings of the short-term rate and volatility, respectively. To compute them, I first use the estimates reported in Longstaff and Schwartz (1993, exhibit 3 p. 10), which are $\alpha=0.001149, \beta=0.1325, \delta=0.05658, \nu=0.335$. In table 3, "case A", the previous figures are used to compute four important coefficients of system (9); "case B", instead, reports coefficients computed using ad hoc chosen coefficients: $\alpha=0.10, \beta=0.13, \delta=0.55, \nu=0.33$; the column corresponding to "Eigenvalues" reports the eigenvalues of matrix $\left(\begin{array}{cc}-b_{1} & b_{2} \\ \varphi_{1} & -\varphi_{2}\end{array}\right)$.

\section{Table 3}

\begin{tabular}{|c|c|c|c|c|c|}
\hline & $b_{1}$ & $b_{2}$ & $\varphi_{1}$ & $\varphi_{2}$ & eigenvalues \\
\hline case A & $5.4145 \cdot 10^{-2}$ & -2.1197 & $3.227 \cdot 10^{-4}$ & 0.3374 & $-5.658 \cdot 10^{-2} ;-0.3349$ \\
\hline case B & 1.2833 & 7.3333 & $-9.5333 \cdot 10^{-2}$ & -0.4033 & $-0.5497 ;-0.3302$ \\
\hline
\end{tabular}

Figure 3 depicts the two factor loadings in these two cases. Consistently with proposition $5, C \leq 0$ at short maturity dates. In case $\mathrm{B}, C>0$ at medium-long maturity dates. To see this with the methods of section 4 , notice that for model (9), eq. (C1) is

$$
h^{2}=-u+\varphi_{1} u_{2}+\psi_{1} u_{22}=\left(\varphi_{1} D+\psi_{1} D^{2}-1\right) u,
$$

and since $\lim _{T \downarrow t} D(T-t)=0, u_{1}$ can never be positive at short maturity dates. Because $\psi_{1}<0$, the previous formula reveals that a necessary condition for $C=w^{2} / u=$ $\int h^{2} / u>0$ at longer maturity dates is that $\varphi_{1} \cdot u_{2}>0$. In case $\mathrm{B}, \varphi_{1}$ and $u_{2}$ are both negative, and as figure 3 shows, $u_{1}$ becomes positive at medium-long maturity dates. In case A, $\varphi_{1}$ and $u_{2}$ are both positive; given the small value of $\varphi_{1}$, however, bond prices are always negatively related to the short-term rate in case A. More on the role of $\varphi_{1}$ in this kind of models below.

To understand the sign of $u_{2}$ in the two cases, consider condition (11) and proposition 6 . In case $\mathrm{A}, u_{1}$ is always negative and $b_{2}<0$. Furthermore, bond prices are convex in the short-term rate because the LS model is affine: by condition (11), bond prices can never be decreasing in volatility. In case $\mathrm{B}, b_{2}>0$; in addition, slope effects dominate convexity effects at short maturity dates. By proposition 6 , bond prices are then decreasing in 

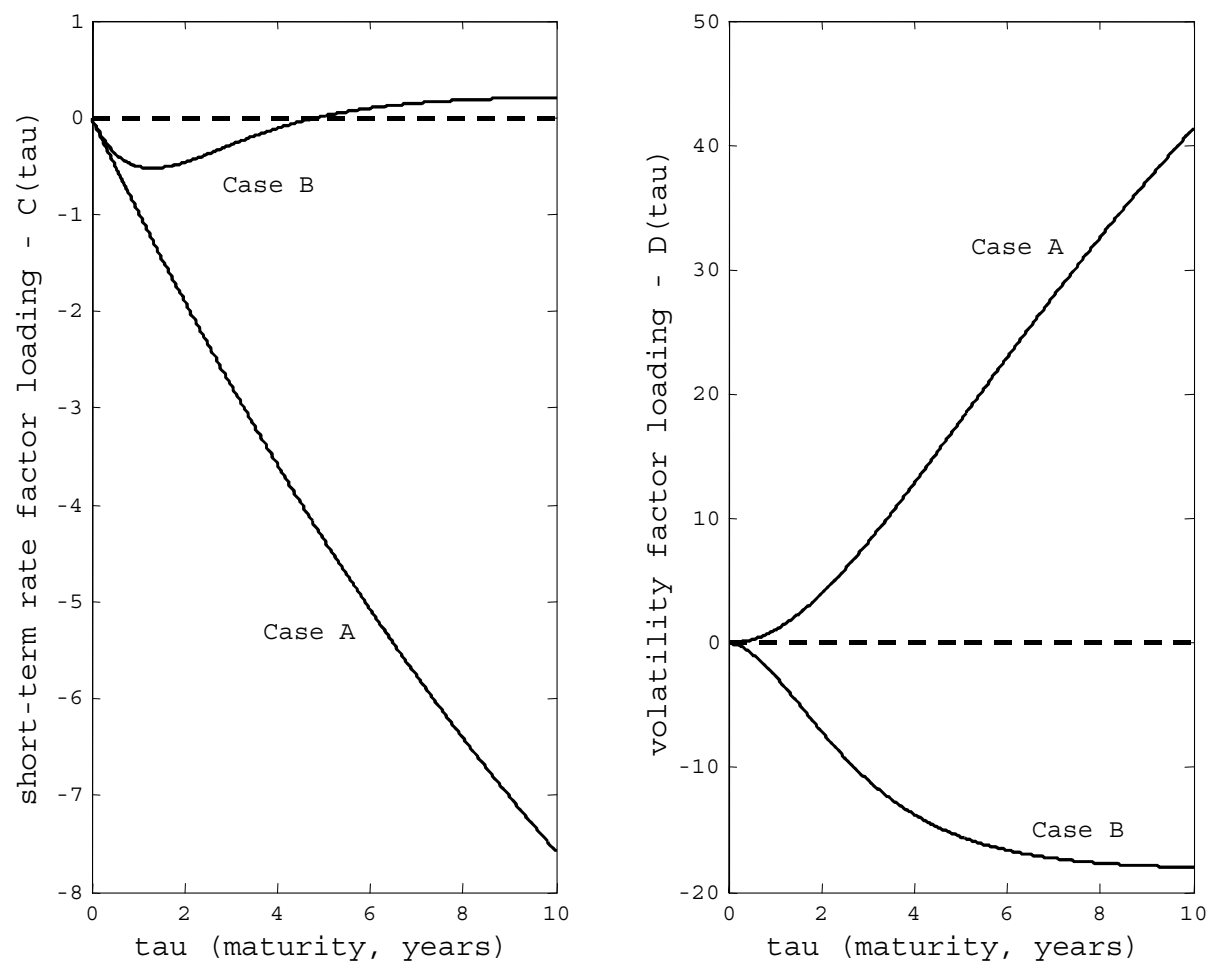

Figure 3 - Factor loadings in model (9). The two graphs depict factor loadings for the pricing equation (C3). The graph at left depicts the short-term rate factor loading $C(\tau)$ under case $\mathrm{A}$ and case $\mathrm{B}$ of table 3 . The graph at right shows the volatility factor loading $D(\tau)$, also computed under case A and case $\mathrm{B}$ of table 3 . When $C(\tau)$ is negative, bond prices are negatively related to random changes of the short-term rate. When $D(\tau)$ is positive, bond prices are positively related to random volatility changes.

volatility at short maturity dates. Given the parameter values of table 3, it also turns out that slope effects dominate convexity effects even at long maturity dates, thus making bond prices react negatively to volatility changes even at long maturity dates.

To examine further the role of the volatility drift function in explaining how bond prices react positively to the short-term rate at medium-long maturity dates, consider a toy model with $\psi_{1}(r, y)=0$ and a semilinear volatility drift function:

$$
\varphi(r, y)=\varphi_{1} r+\widehat{\varphi}(y),
$$

where $\varphi_{1}$ is a constant and $\widehat{\varphi}$ is a well-defined function. By lemma A3,

$$
\begin{aligned}
w^{2}\left(\vartheta_{(2)}(\tau), \phi_{(2)}(\tau), \tau, T ; \varphi_{1}\right)= & -\mathbb{E}\left\{\int_{t}^{T} \kappa^{2}\left(t, \tau ; \varphi_{1}\right) \cdot u\left(\vartheta_{(2)}(\tau), \phi_{(2)}(\tau), \tau, T ; \varphi_{1}\right) \cdot d \tau\right\} \\
& +\varphi_{1} \cdot \mathbb{E}\left\{\int_{t}^{T} \kappa^{2}\left(t, \tau ; \varphi_{1}\right) \cdot w^{1}\left(\vartheta_{(2)}(\tau), \phi_{(2)}(\tau), \tau, T ; \varphi_{1}\right) \cdot d \tau\right\}
\end{aligned}
$$


where I have emphasized the dependence of the various functions on $\varphi_{1}$. A sufficient condition for $w^{2}>0$ at an arbitrarily small but strictly positive maturity date $T$, is that $\left|\varphi_{1}\right|$ may be increased without bounds and the remaining parameters of the model may at the same time be smoothly changed in such a way that:

$$
\lim _{\left|\varphi_{1}\right| \rightarrow \infty}\left|\Phi\left(\varphi_{1}\right)\right|<\infty
$$

where

$$
\Phi\left(\varphi_{1}\right) \equiv \frac{\mathbb{E}\left\{\int_{t}^{T} \kappa^{2}\left(t, \tau ; \varphi_{1}\right) \cdot u\left(\vartheta_{(2)}(\tau), \phi_{(2)}(\tau), \tau, T ; \varphi_{1}\right) \cdot d \tau\right\}}{\mathbb{E}\left\{\int_{t}^{T} \kappa^{2}\left(t, \tau ; \varphi_{1}\right) \cdot w^{1}\left(\vartheta_{(2)}(\tau), \phi_{(2)}(\tau), \tau, T ; \varphi_{1}\right) \cdot d \tau\right\}},
$$

a continuous function. Indeed, if (C4) holds, $\Phi$ has at least one fixed point because

$$
\lim _{\left|\varphi_{1}\right| \rightarrow 0+}\left|\Phi\left(\varphi_{1}\right)\right|<\infty .
$$

Assume the existence of a single fixed point (the following reasoning can be generalized to the presence of multiple fixed points according to the strategy of proofs of section B). One can then take as a critical value of $\varphi_{1}$ the fixed point:

$$
\varphi_{1}^{*}=\Phi\left(\varphi_{1}^{*}\right)
$$

If $\varphi_{1}^{*}<0$ (resp. $>0$ ), the bond price is an increasing function of the short-term rate at any arbitrarily small maturity date $\widetilde{T}$ for any $\varphi_{1}<\varphi_{1}^{*}$ (resp. $>\varphi_{1}^{*}$ ). Of course, all such $\varphi_{1}$ will be functions of $x, s, \widetilde{T}$.

Proof of proposition $6 .{ }^{19}$ I only provide the proof of the weak term-structure augmenting volatility property, the proof of the weak term-structure decreasing volatility property being nearly identical. Suppose that $w^{3} \leq 0$ and $w^{2}<0$ for all $T>t$. In this case the proof is complete by lemma A3. Now suppose that $w^{3}>0$ for all $T \in\left(t, T^{* *}\right)$ where $T^{* *}<\infty$ is given, but still assume that $w^{2}<0$ for all $T \in\left(t, T^{* *}\right)$. First, I provide a heuristic proof that allows one to develop intuition on the main features of prop. 6 and that is based on some regularity conditions. Then I prove prop. 6 in full generality. Suppose then that $\sigma_{2}(x, s)>0$ (the case $\sigma_{2}(x, s)<0$ is treated similarly), and that for any $T \in\left(t, T^{* *}\right), \frac{u_{11}}{-u_{1}}(x, s, t, T)$ is $((x, s)-)$ uniformly bounded. Then, there exists a continuous function $K(t, \cdot)$ such that $\forall(r, y) \in \mathbb{R}_{++} \times \mathbb{R}, \frac{u_{11}}{-u_{1}}(r, y, \tau, T)$ $\leq K(\tau, T)$ and that, by lemma $\mathrm{A} 5$,

$$
\lim _{T \downarrow t} K(t, T)=0 .
$$

\footnotetext{
${ }^{19}$ An alternative proof based on the derivative of the yield curve at the origin is available upon request from the author. The method of such a proof, however, can not be used to show any of the other results appearing in this paper. Perhaps its most severe limitation is that it does not make emerge pieces of information concerning slope and convexity issues, which instead play quite an important role in ensuring "strong" versions of the term-structure augmenting (decreasing) volatility property by the maximum principle: see condition (11), proposition 8, appendices B, C, D and F, and all of the model examples worked out in sections 4,5 and 7 . Finally, even within the context of proposition 6 , such an alternative method of proof does not allow us to develop intuition on the origins of the phenomenon described in this proposition (see, e.g., the discussion in section 2 on the connection of bond prices convexity to the Rothschild-Stiglitz-Jagannathan theory).
} 
Next, suppose that there exists a maturity date within which

$$
\left\{\begin{array}{l}
F_{1}(x, s, t, \tau, T) \equiv-\mathbb{E}\left\{\kappa^{1}(t, \tau) \cdot \sigma_{2}\left(\vartheta_{(1)}(\tau), \phi_{(1)}(\tau)\right) \cdot u_{1}\left(\vartheta_{(1)}(\tau), \phi_{(1)}(\tau), \tau, T\right)\right\} \\
F_{2}(x, s, t, \tau, T) \equiv \frac{\mathbb{E}\left\{\kappa^{1}(t, \tau) \cdot u_{1}\left(\vartheta_{(1)}(\tau), \phi_{(1)}(\tau), \tau, T\right) \cdot b_{2}\left(\vartheta_{(1)}(\tau), \phi_{(1)}(\tau)\right)\right\}}{\mathbb{E}\left\{\kappa^{1}(t, \tau) \cdot u_{1}\left(\vartheta_{(1)}(\tau), \phi_{(1)}(\tau), \tau, T\right) \cdot \sigma_{2}\left(\vartheta_{(1)}(\tau), \phi_{(1)}(\tau)\right)\right\}}
\end{array}\right.
$$

are well-defined. We have:

$$
\forall T \in\left(t, T^{* *}\right), \quad w^{1}(x, s, t, T) \leq \int_{t}^{T} F_{1}(x, s, t, \tau, T) \cdot\left\{K(\tau, T)-F_{2}(x, s, t, \tau, T)\right\} d \tau,
$$

and by applying the same kind of arguments used to derive (B4),

$$
\begin{aligned}
\lim _{T \downarrow t} F_{2}(x, s, t, \tau, T) & =\lim _{T \downarrow t} \frac{\mathbb{E}\left\{\kappa^{1}(t, \tau) \cdot u_{1}\left(\vartheta_{(1)}(\tau), \phi_{(1)}(\tau), \tau, T\right) \cdot b_{2}\left(\vartheta_{(1)}(\tau), \phi_{(1)}(\tau)\right)\right\}}{\mathbb{E}\left\{\kappa^{1}(t, \tau) \cdot u_{1}\left(\vartheta_{(1)}(\tau), \phi_{(1)}(\tau), \tau, T\right) \cdot \sigma_{2}\left(\vartheta_{(1)}(\tau), \phi_{(1)}(\tau)\right)\right\}} \\
& =\frac{b_{2}(x, s)}{\sigma_{2}(x, s)}>0 .
\end{aligned}
$$

Combining (C6) with (C8) shows that there exists a $T^{*}$ depending on $x, s$ such that $T^{*}<T^{* *}$ and $\forall \tau \in\left[t, T^{*}\right], K\left(\tau, T^{*}\right) \leq F_{2}\left(x, s, t, \tau, T^{*}\right)$; the result then follows by $(\mathrm{C} 7)$.

The previous case makes clearly see how lemma A5 (slope effects dominate convexity effects at short-maturity dates) is to be related to the bond price reaction to random volatility changes at short-maturity dates. When $\frac{u_{11}}{-u_{1}}(x, s, t, T)$ is not $((x, s)-)$ uniformly bounded and the regularity conditions underlying $(\mathrm{C} 7)$ are not easy to check (these conditions are always satisfied by affine models), the proof is similar to the the proof of (B4) and so here it will be sketchy. For each $T \in\left(t, T^{* *}\right)$, rewrite $w^{1}$ in lemma A1 as:

$w^{1}(x, s, t, T)=\mathbb{E}\left\{\int_{t}^{T}\left(\kappa^{1}\left(-u_{1}\right)\right)(\tau) d \tau\right\}\left\{\frac{\mathbb{E}\left\{\int_{t}^{T}\left(\kappa^{1} \sigma_{2} u_{11}\right)(\tau) d \tau\right\}}{\mathbb{E}\left\{\int_{t}^{T}\left(\kappa^{1}\left(-u_{1}\right)\right)(\tau) d \tau\right\}}-\frac{\mathbb{E}\left\{\int_{t}^{T}\left(\kappa^{1} b_{2}\left(-u_{1}\right)\right)(\tau) d \tau\right\}}{\mathbb{E}\left\{\int_{t}^{T}\left(\kappa^{1}\left(-u_{1}\right)\right)(\tau) d \tau\right\}}\right\}$

and conclude by showing that $\lim _{T \downarrow t} \mathbb{E}\left\{\int_{t}^{T}\left(\kappa^{1} \sigma_{2} u_{11}\right)(\tau) d \tau\right\} / \mathbb{E}\left\{\int_{t}^{T}\left(\kappa^{1}\left(-u_{1}\right)\right)(\tau) d \tau\right\}$ $=0$ and $\lim _{T \downarrow t} \mathbb{E}\left\{\int_{t}^{T}\left(\kappa^{1} b_{2}\left(-u_{1}\right)\right)(\tau) d \tau\right\} / \mathbb{E}\left\{\int_{t}^{T}\left(\kappa^{1}\left(-u_{1}\right)\right)(\tau) d \tau\right\}=b_{2}(x, s)>0$ [use: 1) the L'Hôpital's rule; 2) Lebesgue's dominated convergence theorem; 3) the Liebnitz's rule; 4) $\lim _{T \downarrow t} \frac{\partial u_{1}}{\partial T}=-1, \lim _{T \downarrow t} \frac{\partial u_{11}}{\partial T}=0$; and 5) lemma A4], and noting that there exists a maturity date within which $\mathbb{E}\left\{\int_{t}^{T}\left(\kappa^{1}\left(-u_{1}\right)\right)(\tau) d \tau\right\} \geq 0$, with equality as $T \downarrow t$.

Finally, relax the assumption that $w^{2}<0$ for all $T \in\left(t, T^{* *}\right)$. By proposition 5 , there still exists a maturity date $T_{* *}(x, s)$ within which $\mathbb{E}\left\{\int_{t}^{T}\left(\kappa^{1}\left(-u_{1}\right)\right)(\tau) d \tau\right\} \geq 0$ (with equality as $T \downarrow t$ ), and the proof is complete by repeating the same arguments produced before. $\|$

Proof of relations (12). Let $\nu \equiv \partial \vartheta / \partial x$ denote the first partial of the short-term rate flow $\vartheta(\tau)$ with respect to the initial condition $\vartheta(t)=x$; a simple computation reveals that 
$\nu(\tau)=\exp (-\theta(\tau-t))$, which substituted in (5) and (6) leaves $u_{1}(x, s, t, T) / u(x, s, t, T)=$ $-(1-\exp (-\theta(T-t))) / \theta$ and $u_{11}(x, s, t, T) / u(x, s, t, T)=((1-\exp (-\theta(T-t))) / \theta)^{2}$. Substituting such expressions of $u_{1}$ and $u_{11}$ into eq. (7) enables one to conclude that $u_{2}(x, s, t, T)<0$ whenever $\lambda_{1} \geq\{1-\exp (-\theta(T-t))\} /(2 \theta)$. Relations (12) then follow immediately. \|

\section{Appendix D: proofs for section 5}

Lemma D1 (Slope effects dominate cross-unobserved factors effects at short maturity dates). For each $(x, s, c) \in \mathbb{R}_{++} \times \mathbb{R} \times \mathbb{R}, \lim _{T \downarrow t} \frac{u_{1 j}}{u_{1}}(x, s, c, t, T)=0, j=1,2,3$.

Proof. I use the same strategy of proof as in lemma A5. Consider the limit of $u_{12} / u_{1}$ :

$$
\begin{aligned}
& \lim _{T \downarrow t} \frac{u_{12}}{u_{1}}(x, s, c, t, T) \\
= & \lim _{T \downarrow t} \frac{\mathbb{E}\left\{\left[\int_{t}^{T} \frac{\partial^{2} \vartheta}{\partial x \partial s}(\tau) d \tau-\left(\int_{t}^{T} \frac{\partial \vartheta}{\partial x}(\tau) d \tau\right)\left(\int_{t}^{T} \frac{\partial \vartheta}{\partial s}(\tau) d \tau\right)\right] \cdot \exp \left(-\int_{t}^{T} \vartheta(\tau) d \tau\right)\right\}}{\mathbb{E}\left\{\left(\int_{t}^{T} \frac{\partial \vartheta}{\partial x}(\tau) d \tau\right) \cdot \exp \left(-\int_{t}^{T} \vartheta(\tau) d \tau\right)\right\}} \\
= & \lim _{T \downarrow t} \frac{\mathbb{E}\left\{\left[\frac{1}{T-t} \int_{t}^{T} \frac{\partial^{2} \vartheta}{\partial x \partial s}(\tau) d \tau-(T-t)\left(\frac{1}{T-t} \int_{t}^{T} \frac{\partial \vartheta}{\partial x}(\tau) d \tau\right)\left(\frac{1}{T-t} \int_{t}^{T} \frac{\partial \vartheta}{\partial s}(\tau) d \tau\right)\right] \cdot \exp \left(-\int_{t}^{T} \vartheta(\tau) d \tau\right)\right\}}{\mathbb{E}\left\{\left(\frac{1}{T-t} \int_{t}^{T} \frac{\partial \vartheta}{\partial x}(\tau) d \tau\right) \cdot \exp \left(-\int_{t}^{T} \vartheta(\tau) d \tau\right)\right\}},
\end{aligned}
$$

and, again, the result follows from the Lebesgue's dominated convergence theorem, since $\int \frac{\partial^{2} \vartheta}{\partial x \partial s}, \int \frac{\partial \vartheta}{\partial x}$ and $\int \frac{\partial \vartheta}{\partial s}$ are Riemann integrals and then, as $T \downarrow t, \frac{1}{T-t} \int_{t}^{T} \frac{\partial \vartheta}{\partial x}(\tau) d \tau \rightarrow \frac{\partial \vartheta}{\partial x}(t)$, $\frac{1}{T-t} \int_{t}^{T} \frac{\partial^{2} \vartheta}{\partial x \partial s}(\tau) d \tau \rightarrow \frac{\partial^{2} \vartheta}{\partial x \partial s}(t)$ and $\frac{1}{T-t} \int_{t}^{T} \frac{\partial \vartheta}{\partial s}(\tau) d \tau \rightarrow \frac{\partial \vartheta}{\partial s}(t)$, where $\frac{\partial \vartheta}{\partial x}(t)=1, \frac{\partial^{2} \vartheta}{\partial x \partial s}(t)=0$ and $\frac{\partial \vartheta}{\partial s}(t)=0$ by a straightforward generalization of lemma A4. The proof for the other partial derivatives is nearly identical. \|

Proof of proposition $\%$. The proof utilizes the same arguments used during the proof of proposition 6 , so it will be sketchy. Let the short-term rate be solution of system (14). Denote the rational price function as $u(r, y, \ell, \tau, T)$. Under the conditions mentioned in section $1, u$ is then the solution of the following partial differential equation:

$$
\left\{\begin{array}{l}
0=\left(\frac{\partial}{\partial \tau}+L-r\right) u(r, y, \ell, \tau, T), \forall(r, y, \ell, \tau) \in \mathbb{R}_{++} \times \mathbb{R} \times \mathbb{R} \times[t, T) \\
u(r, y, \ell, T, T)=1, \forall(r, y, \ell) \in \mathbb{R}_{++} \times \mathbb{R} \times \mathbb{R}
\end{array}\right.
$$

where

$L u=b u_{1}+\sigma u_{11}+\varphi u_{2}+\psi u_{22}+\varepsilon u_{3}+\pi u_{33}+\sum_{j=1}^{3} \sigma^{(j)} \psi^{(j)} \cdot u_{12}+\sum_{j=1}^{3} \sigma^{(j)} \pi^{(j)} \cdot u_{13}+\sum_{j=1}^{3} \psi^{(j)} \pi^{(j)} \cdot u_{23}$.

Differentiating eq. (D1) with respect to $r$ and $y$ reveals that $w^{1} \equiv u_{2}$ and $w^{2} \equiv u_{1}$ 
satisfy the following partial differential equations:

$$
\left\{\begin{aligned}
& 0=\left(\frac{\partial}{\partial \tau}+L^{i}-k^{i}\right) w^{i}(\vartheta, \phi, \chi, \tau, T)+h^{i}(\vartheta, \phi, \chi, \tau, T), \\
& \forall(\vartheta, \phi, \chi, \tau) \in \mathbb{R}_{++} \times \mathbb{R} \times \mathbb{R} \times[t, T) \\
& w^{i}(\vartheta, \phi, \chi, T, T)=0, \quad \forall(\vartheta, \phi, \chi) \in \mathbb{R}_{++} \times \mathbb{R} \times \mathbb{R}
\end{aligned}\right.
$$

where $\chi$ denotes the stochastic flow of $\ell$, functions $h^{i}$ and $k^{i}$ are given by

$$
\left\{\begin{array}{l}
h^{1}=b_{2} u_{1}+\sigma_{2} u_{11}+\varepsilon_{2} u_{3}+\pi_{2} u_{33}+\left(\sum_{j=1}^{3} \frac{\partial}{\partial y} \sigma^{(j)} \pi^{(j)}\right) \cdot u_{13} \\
h^{2}=\varphi_{1} u_{2}+\psi_{1} u_{22}+\varepsilon_{1} u_{3}+\pi_{1} u_{33}+\left(\sum_{j=1}^{3} \frac{\partial}{\partial r} \psi^{(j)} \pi^{(j)}\right) \cdot u_{23}-u
\end{array}\right.
$$

and

$$
\left\{\begin{array}{l}
k^{1}(\vartheta, \phi, \chi)=\vartheta-\varphi_{2}(\vartheta, \phi, \chi) \\
k^{2}(\vartheta, \phi, \chi)=\vartheta-b_{1}(\vartheta, \phi, \chi)
\end{array}\right.
$$

and operators $L^{i}$ satisfy

$$
\left\{\begin{aligned}
L^{1} w^{1}= & \left(b+\sum_{j=1}^{3} \frac{\partial}{\partial y} \sigma^{(j)} \psi^{(j)}\right) w_{1}^{1}+\left(\varphi+\psi_{2}\right) w_{2}^{1}+\left(\varepsilon+\sum_{j=1}^{3} \frac{\partial}{\partial y} \psi^{(j)} \pi^{(j)}\right) w_{3}^{1}+\sigma w_{11}^{1} \\
& +\psi w_{22}^{1}+\pi w_{33}^{1}+\sum_{j=1}^{3} \sigma^{(j)} \psi^{(j)} w_{12}^{1}+\sum_{j=1}^{3} \sigma^{(j)} \pi^{(j)} w_{13}^{1}+\sum_{j=1}^{3} \psi^{(j)} \pi^{(j)} w_{23}^{1} \\
L^{2} w^{2}= & \left(b+\sigma_{1}\right) w_{1}^{2}+\left(\varphi+\sum_{j=1}^{3} \frac{\partial}{\partial r} \sigma^{(j)} \psi^{(j)}\right) w_{2}^{2}+\left(\varepsilon+\sum_{j=1}^{3} \frac{\partial}{\partial r} \sigma^{(j)} \pi^{(j)}\right) w_{3}^{2}+\sigma w_{11}^{2} \\
& +\psi w_{22}^{2}+\pi w_{33}^{2}+\sum_{j=1}^{3} \sigma^{(j)} \psi^{(j)} w_{12}^{2}+\sum_{j=1}^{3} \sigma^{(j)} \pi^{(j)} w_{13}^{2}+\sum_{j=1}^{3} \psi^{(j)} \pi^{(j)} w_{23}^{2}
\end{aligned}\right.
$$

By using the same arguments produced to show lemma A3 and proposition 5, one shows that the system in (D2) obtained with $i=2$ implies that there exists a maturity date $T_{*}$ depending on $(x, s, c)$ such that $u_{1}<0$ for each $T \leq T_{*}$. The result then follows by lemma D1 (bounded case) and/or by applying the same arguments produced to show lemma A3 and proposition 6 to the first equation in (D2) (unbounded case). The proof for factor $\ell$ is entirely analogous. $\|$

Proof of proposition 8. Follows from eq. (D2). ॥

Analysis of models (13) and (16). First I show the claim in the main text that relation (18) is sufficient to guarantee that bond prices are increasing in volatility at any finite maturity date whenever $\lambda_{r}+\lambda_{v} \sigma_{r v} \eta^{2}>0, \lambda_{r} \sigma_{\theta r}<0$ and $\kappa>\nu$ in model (13). According to proposition 8 , it is sufficient to show that for any $(r, y, \ell, \tau) \in \mathbb{R} \times \mathbb{R} \times \mathbb{R} \times[t, T)$,

$$
h^{1}=-\left(\lambda_{r}+\lambda_{v} \sigma_{r v} \eta^{2}\right) u_{1}+\frac{1+\sigma_{r v}^{2} \eta^{2}}{2} u_{11}-\lambda_{r} \sigma_{\theta r} u_{3}+\frac{\sigma_{\theta r}^{2}}{2} u_{33}+\sigma_{\theta r} u_{13}>0 .
$$


Let $m_{\vartheta, x} \equiv \partial \vartheta / \partial x$ and $m_{\vartheta, c} \equiv \partial \vartheta / \partial c$ the sensitivity of the interest rate flow to initial conditions $r(\tau)=x$ and $\chi(\tau)=c$. We have: $u_{1}(\cdot)=-\left(\int_{\tau}^{T} m_{\vartheta, x}(v) d v\right) u(\cdot), u_{11}(\cdot)=$ $\left(\int_{\tau}^{T} m_{\vartheta, x}(v) d v\right)^{2} u(\cdot), u_{3}(\cdot)=-\left(\int_{\tau}^{T} m_{\vartheta, c}(v) d v\right) u(\cdot), u_{33}(\cdot)=\left(\int_{\tau}^{T} m_{\vartheta, c}(v) d v\right)^{2} u(\cdot), u_{13}(\cdot)=$ $\left(\int_{\tau}^{T} m_{\vartheta, x}(v) d v\right)\left(\int_{\tau}^{T} m_{\vartheta, c}(v) d v\right) u(\cdot)$, where

$$
\left\{\begin{array}{l}
\int_{\tau}^{T} m_{\vartheta, x}(v) d v=\frac{1-\exp (-\kappa(T-\tau))}{\kappa} \\
\int_{\tau}^{T} m_{\vartheta, c}(v) d v=\left(\int_{\tau}^{T} m_{\vartheta, x}(u) d u\right) \cdot \frac{\kappa}{\kappa-\nu} \cdot\left(\frac{1-\exp (-\nu(T-\tau))}{1-\exp (-\kappa(T-\tau))} \frac{\kappa}{\nu}-1\right)
\end{array}\right.
$$

Relation (D3) can thus be written as:

$$
\begin{aligned}
\frac{h^{1}(\tau, T)}{u(r, y, \ell, \tau, T)}= & \widetilde{h}^{1}(\tau, T) \\
\equiv & \left(\int_{\tau}^{T} m_{\vartheta, x}(v) d v\right) \cdot\left\{\lambda_{r}+\lambda_{v} \sigma_{r v} \eta^{2}+\lambda_{r} \sigma_{\theta r} \frac{\kappa}{\kappa-\nu}\left(\frac{1-\exp (-\nu(T-\tau))}{1-\exp (-\kappa(T-\tau))} \frac{\kappa}{\nu}-1\right)\right\} \\
& \left.+\frac{\sigma_{r v}^{2} \eta^{2}}{2}\left(\int_{\tau}^{T} m_{\vartheta, x}(v) d v\right)^{2}+\frac{1}{2}\left\{\int_{\tau}^{T} m_{\vartheta, x}(v) d v+\sigma_{\theta r} \int_{\tau}^{T} m_{\vartheta, c}(v) d v\right)\right\}^{2}
\end{aligned}
$$

When $\lambda_{r}+\lambda_{v} \sigma_{r v} \eta^{2}>0$ and $\lambda_{r} \sigma_{\theta r}<0$, a sufficient condition for $h^{1}>0$ for all $\tau \in[t, T)$ is then that

$$
\frac{\lambda_{r}+\lambda_{v} \sigma_{r v} \eta^{2}}{-\lambda_{r} \sigma_{\theta r}}>\frac{\kappa}{\kappa-\nu}\left(\frac{1-\exp (-\nu(T-\tau))}{1-\exp (-\kappa(T-\tau))} \frac{\kappa}{\nu}-1\right) .
$$

Now, function $f(T-\tau) \equiv(1-\exp (-\nu(T-\tau))) /(1-\exp (-\kappa(T-\tau))), \tau$ varying, is continuous in $[t, T)$, with $f(T-\tau)<1$ for any $\tau \in[t, T)$ and $\lim _{\tau \uparrow T} f(T-\tau)=\nu / \kappa<1$, which shows the sufficiency of relation (18) for $h^{1}>0$. Notice also that the second relation in (D4) reveals that $u_{3}<0$ for any finite maturity date.

When relation (18) does not hold, eq. (D5) may be used to develop a condition for bond prices to react negatively to volatility at longer maturity dates. By a straightforward extension of lemma A1, and lemma D1, $u_{2}(x, s, c, t, T)=\mathbb{E}\left\{\int_{t}^{T} \kappa^{1}(t, \tau)\right.$. $\left.h^{1}\left(\vartheta_{(1)}(\tau), \phi_{(1)}(\tau), \chi_{(1)}(u), \tau, T\right) d \tau\right\}$, where $\kappa^{1}(t, \tau)=\exp \left(-\int_{t}^{\tau}\left(\vartheta_{(1)}(u)-\varphi_{2}\left(\vartheta_{(1)}(u)\right.\right.\right.$, $\left.\left.\left.\phi_{(1)}(u), \chi_{(1)}(u)\right)\right) d u\right) \in(0,1]$ for $\tau \in[t, T]$, and $\left(\vartheta_{(1)}, \phi_{(1)}, \chi_{(1)}\right)$ is solution of the same diffusion process as (13), except that the drifts are as in operator $L^{1}$ in eq. (D2). Furthermore, the parameters estimates reported by Dai and Singleton (2000) are such that $\exists \tau^{*}: \forall T>\tau^{*}, \widetilde{h}^{1}(\tau, T)<0$ for all $\tau \in\left[t, \tau^{*}\right]$, and $\widetilde{h}^{1}(\tau, T)>0$ for all $\tau \in\left[\tau^{*}, T\right]$. Let $\zeta(x, s, c, t, \tau, T) \equiv \mathbb{E}\left\{\kappa^{1}(t, \tau) \cdot u\left(\vartheta_{(1)}(\tau), \phi_{(1)}(\tau), \chi_{(1)}(u), \tau, T\right)\right\} \in(0,1]$. We have:

$$
u_{2}(x, s, c, t, T)=\int_{t}^{\tau^{*}} \zeta(x, s, c, t, \tau, T) \cdot \widetilde{h}^{1}(\tau, T) d \tau+\int_{\tau^{*}}^{T} \zeta(x, s, c, t, \tau, T) \cdot \widetilde{h}^{1}(\tau, T) d \tau .
$$

Therefore, $u_{2}<0$ at longer maturity dates whenever:

$$
\int_{\tau^{*}}^{T} \widetilde{h}^{1}(\tau, T) d \tau<\frac{\min _{\tau \in\left[t, \tau^{*}\right]} \zeta(x, s, c, t, \tau, T)}{\mathbb{E}\left\{\kappa^{1}\left(t, \tau^{*}\right)\right\}} \cdot \int_{t}^{\tau^{*}}\left\{-\widetilde{h}^{1}(\tau, T)\right\} d \tau .
$$


To illustrate, eq. (D6) for $T=2$ holds when $0.2981<\min _{\tau \in[0,1.25]} \bar{\zeta}(x, s, c, 0, \tau, 2)$, where $\bar{\zeta} \equiv \zeta e^{-\left(\mu+\lambda_{v} \eta^{2}\right)}$. As an example, by taking $(x, s, c)=(0.1041,0.1134,0.0151)$ (which are the values of $r, y$ and $\ell$ under which the drift functions of system (14) are zeroed), I find that $\min _{\tau \in[0,1.25]} \bar{\zeta}(x, s, c, 0, \tau, 2)=0.8132$ after a straightforward numerical analysis in which $\zeta$ was computed as: $\bar{\zeta}(x, s, c, 0, \tau, T)=\mathbb{E}\left\{\exp \left(-\int_{0}^{\tau} \vartheta_{(1)}(u) d u\right) \mathbb{E}\left\{\exp \left(-\int_{\tau}^{T} \vartheta(u) d u\right)\right.\right.$ $\left.\left./(\vartheta, \phi, \chi)(\tau)=\left(\vartheta_{(1)}, \phi_{(1)}, \chi_{(1)}\right)(\tau)\right\} /\left(\vartheta_{(1)}, \phi_{(1)}, \chi_{(1)}\right)(0)=x, s, c\right\}=\mathbb{E}\left\{\exp \left(\int_{0}^{\tau}(\vartheta(u)-\right.\right.$ $\left.\left.\vartheta_{(1)}(u)\right) d u\right) \cdot \exp \left(-\int_{0}^{T} \vartheta(u) d u\right) /$ initial state $\left.=x, s, c\right\}$.

Finally, I show the claim in the main text that relation (17) is sufficient to guarantee that bond prices are decreasing in volatility at any finite maturity whenever $\kappa_{r v}+\lambda_{r}+$ $\lambda_{v} \sigma_{r v} \eta^{2}<0$ in model (16) (see, also, footnote 16). First, I show that $u_{3}<0$. It suffices to apply condition (15) for factor $\ell$ and conclude that for any $(r, y, \ell, \tau) \in \mathbb{R} \times \mathbb{R} \times \mathbb{R} \times[t, T)$, $u_{3}(r, y, \ell, \tau)<0$ whenever $\kappa u_{1}(r, y, \ell, \tau)<0$. But $\kappa>0$, and $\kappa u_{1}<0$ because $u_{1}<0$ by an application of (D2) for $i=2$. To show that $u_{2}<0$, use proposition 8 and note that it is sufficient to show that for any $(r, y, \ell, \tau) \in \mathbb{R} \times \mathbb{R} \times \mathbb{R} \times[t, T)$,

$$
-\left(\kappa_{r v}+\lambda_{r}+\lambda_{v} \sigma_{r v} \eta^{2}\right) u_{1}+\frac{1+\eta^{2} \sigma_{r v}}{2} u_{11}-\kappa_{\theta v} u_{3}<0 .
$$

Because $\kappa_{\theta v}<0$ and $u_{3}<0$, it is sufficient to show that $-\left(\kappa_{r v}+\lambda_{r}+\lambda_{v} \sigma_{r v} \eta^{2}\right) u_{1}+$ $\left(\left(1+\eta^{2} \sigma_{r v}\right) / 2\right) u_{11}<0$. But again, $u_{1}(\cdot)=-\left(\int_{\tau}^{T} m_{\vartheta, x}(v) d v\right) u(\cdot)$ and $u_{11}(\cdot)=$ $\left(\int_{\tau}^{T} m_{\vartheta, x}(v) d v\right)^{2} u(\cdot)$, where $\left.\int_{\tau}^{T} m_{\vartheta, x}(v) d v\right)=\{1-\exp (-\kappa(T-\tau))\} / \kappa$, which implies that (D7) holds for any $(r, y, \ell, \tau) \in \mathbb{R} \times \mathbb{R} \times \mathbb{R} \times[t, T)$ whenever relation (17) in the main text is true.

\section{Appendix E: proofs for section 6}

Proof of proposition 9. By a standard argument, the following equation will be satisfied by the price function $u(r, \tau, T)$ in the absence of arbitrage opportunities:

$$
\left\{\begin{array}{l}
0=\left(\frac{\partial}{\partial \tau}+L^{J}-r\right) u(r, \tau, T), \forall(r, \tau) \in \mathbb{R}_{++} \times[t, T) \\
u(r, T, T)=1, \forall r \in \mathbb{R}_{++}
\end{array}\right.
$$

where $\frac{\partial}{\partial \tau} \cdot+L^{J}$. is the jump-diffusion infinitesimal generator of (19), with

$$
L^{J} u(r, \tau, T)=L u(r, \tau, T)+v(r) \int_{\operatorname{supp}(\mathcal{S})}\left\{u\left(r+a_{2}(r) \mathcal{S}, \tau, T\right)-u(r, \tau, T)\right\} p(d \mathcal{S}),
$$

and $\frac{\partial}{\partial \tau} \cdot+L \cdot$ is the usual infinitesimal generator for diffusion processes.

Next, differentiate eq. (E1) twice with respect to $r$ to obtain:

$$
\left\{\begin{array}{l}
0=\left(\frac{\partial}{\partial \tau}+L^{J, i}-k^{i}\right) w^{i}(\vartheta, \tau, T)+h^{J, i}(\vartheta, \tau, T), \forall(\vartheta, \tau) \in \mathbb{R}_{++} \times[t, T) \\
w^{i}(\vartheta, T, T)=0, \forall \vartheta \in \mathbb{R}_{++}
\end{array}\right.
$$


where $w^{2} \equiv u_{1}, w^{3} \equiv u_{11}$,

$$
\begin{aligned}
h^{J, 2}(\vartheta, \tau, T)= & -u(\vartheta, \tau, T)+v^{\prime}(\vartheta) \int\left\{u\left(\vartheta+a_{2}(\vartheta) \mathcal{S}, \tau, T\right)-u(\vartheta, \tau, T)\right\} \cdot p(d \mathcal{S}) \\
& +v(\vartheta) \int w^{2}\left(\vartheta+a_{2}(\vartheta) \mathcal{S}, \tau, T\right) \cdot a_{2}^{\prime}(\vartheta) \mathcal{S} \cdot p(d \mathcal{S}), \\
h^{J, 3}(\vartheta, \tau, T)= & -\left(2-b^{J \prime \prime}\right) u_{1}(\vartheta, \tau, T)+v(\vartheta) \int u_{1}\left(\vartheta+a_{2}(\vartheta) \mathcal{S}, \tau, T\right) \cdot a_{2}^{\prime \prime}(\vartheta) \mathcal{S} \cdot p(d \mathcal{S}) \\
& +v(\vartheta) \int w^{3}\left(\vartheta+a_{2}(\vartheta) \mathcal{S}, \tau, T\right) \cdot\left(a_{2}^{\prime}(\vartheta)^{2} \mathcal{S}^{2}+2 a_{2}^{\prime}(\vartheta) \mathcal{S}\right) \cdot p(d \mathcal{S}) \\
& +2 v^{\prime}(\vartheta) \int\left\{u_{1}\left(\vartheta+a_{2}(\vartheta) \mathcal{S}, \tau, T\right) \cdot\left(1+a_{2}^{\prime}(\vartheta) \mathcal{S}\right)-u_{1}(\vartheta, \tau, T)\right\} \cdot p(d \mathcal{S}) \\
& +v^{\prime \prime}(\vartheta) \int\left\{u\left(\vartheta+a_{2}(\vartheta) \mathcal{S}, \tau, T\right)-u(\vartheta, \tau, T)\right\} \cdot p(d \mathcal{S}),
\end{aligned}
$$

and $k^{2}=\vartheta-b^{J \prime}(\vartheta), k^{3}=\vartheta-2 b^{J \prime \prime}(\vartheta)-a^{\prime \prime}(\vartheta)$, with operators $L^{J, i}, i=2,3$, satisfying

$$
L^{J, i} w^{i}(\vartheta, \tau, T)=L^{i} w^{i}(\vartheta, \tau, T)+v(\vartheta) \int\left\{w^{i}\left(\vartheta+a_{2}(\vartheta) \mathcal{S}, \tau, T\right)-w^{i}(\vartheta, \tau, T)\right\} \cdot p(d \mathcal{S}),
$$

where $L^{i}$. are defined similarly as in the proof of lemma A1 in appendix A.

Since $h^{J, 2}(\vartheta, \tau, T)$ tends to minus one as $T$ approach $\tau$, claim a) of proposition 9 follows from an argument nearly identical to the one used to show proposition 5 .

Claim b) follows from rearranging terms in eq. (E2), and claim c) follows because when $v^{\prime}(r)=a_{2}^{\prime}(r)=0$ for all $r \in \mathbb{R}_{++}, h^{J, 2}$ and $h^{J, 3}$ reduce to functionals $h^{2}$ and $h^{3}$ encountered during the proof of lemma A3.

Finally, claim d) is correct because the price difference $\nabla u \equiv u^{A}-u^{B}$ satisfies:

$$
\left\{\begin{array}{l}
0=\left(\frac{\partial}{\partial \tau}+L_{\nabla}^{J}-r\right) \nabla u(r, \tau, T) \\
\quad+\left(v^{A}(r)-v^{B}(r)\right) \int\left\{u^{B}\left(r+a_{2}(r) \mathcal{S}, \tau, T\right)-u^{B}(r, \tau, T)\right\} p(d \mathcal{S}), \forall(r, \tau) \in \mathbb{R}_{++} \times[t, T) \\
\nabla u(r, T, T)=0, \forall r \in \mathbb{R}_{++}
\end{array}\right.
$$

where $L_{\nabla}^{J} \nabla u=b^{J} \nabla u_{1}+a \nabla u_{11}+v^{A}(r) \int\left\{\nabla u\left(r+a_{2}(r) \mathcal{S}\right)-\nabla u(r)\right\} p(d \mathcal{S}) . \|$

A proof of equation (20). Let $\widehat{\tau}$ be the random default time, and define an auxiliary state process $g$ with the property that:

$$
g(\tau)=\left\{\begin{array}{l}
0, \text { if } t \leq \tau<\widehat{\tau} \\
1, \text { otherwise }
\end{array}\right.
$$

In this economy, all relevant information is thus subsumed by the following risk-neutral dynamics:

$$
\left\{\begin{array}{l}
d r(\tau)=b(r(\tau)) d \tau+\sqrt{2 a(r(\tau))} d W(\tau) \\
d g(\tau)=\mathcal{S} \cdot d N(\tau), \text { where } \mathcal{S} \equiv 1 \text { with probability one }
\end{array}\right.
$$


Denote the rational bond price function as $u(r, g, \tau, T) \equiv u^{\operatorname{pre}}(r, \tau, T), \tau \in[t, T]$. By a standard argument, the following equation is satisfied by $u^{\operatorname{pre}}(r, \tau, T)$ in the absence of arbitrage opportunities:

$$
\begin{aligned}
0 & =\left(\frac{\partial}{\partial \tau}+L-r\right) u(r, 0, \tau, T)+v(r) \cdot(u(r, 1, \tau, T)-u(r, 0, \tau, T)) \\
& =\left(\frac{\partial}{\partial \tau}+L-(r+v(r))\right) u(r, 0, \tau, T)+v(r) \bar{u}(\tau), \quad \tau \in[t, T),
\end{aligned}
$$

with the usual boundary condition $u(r, 0, T, T)=1$. The second line of (E3) follows by the definition of the recovery payment and by rearranging terms. Eq. (E3) has exactly the same form as the equations treated in appendix A (see lemma A3). Under the usual regularity conditions, the solution for the pre-default bond price is:

$$
\begin{aligned}
u^{\mathrm{pre}}(x, t, T)= & \mathbb{E}^{*}\left\{\exp \left(-\int_{t}^{T}(r(\tau)+v(r(\tau))) d \tau\right)\right\} \\
& +\mathbb{E}^{*}\left\{\int_{t}^{T} \exp \left(-\int_{t}^{\tau}(r(u)+v(r(u))) d u\right) \cdot v(r(\tau)) \bar{u}(\tau) d \tau\right\},
\end{aligned}
$$

The previous formula is an easy extension of the evaluation formula reported by Duffie, Pan and Singleton (2000, eq. (1.3) p. 1345) in the case of a constant recovery payment. To show that eq. (E4) coincides with the original derivation of Duffie and Singleton (1999, eq. (10) p. 696) (or with the derivation in Lando (1998, example 3.5 p. 107)), i.e. with $(20)$, insert $\bar{u}=(1-l) \cdot u(\cdot, 0, \cdot, \cdot)$ into eq. (E3) to obtain

$$
0=\left(\frac{\partial}{\partial \tau}+L-(r+l(\tau) v(r))\right) u(r, 0, \tau, T), \forall(r, \tau) \in \mathbb{R}_{++} \times[t, T),
$$

with the usual boundary condition, the solution of which is exactly eq. (20). ॥

\section{Appendix F: proofs for section 7}

Proof of inequality (21) for models (13) and (16). I use the same trick used in appendix D for a similar problem (see eq. (D5)), and I conclude that for both models (13) and (16), the l.h.s. of relation (21) is:

$$
\xi \equiv\left(\eta_{A}^{2}-\eta_{B}^{2}\right) \cdot y \cdot\left\{\lambda_{v} \sigma_{r v} u_{1}-\lambda_{v} u_{2}+\frac{1}{2}\left(\sigma_{r v} \int m_{\vartheta, x}+\int m_{\vartheta, s}\right)^{2} u\right\},
$$

where $m_{\vartheta, s}$ is the partial of the short-term rate flow $\vartheta$ with respect to the initial condition of volatility $s$ (clearly, $m_{\vartheta, s}$ is independent of $s$ because models (13) and (16) are affine). Since drift and diffusion functions of $y$ and $\ell$ are independent of $r, u_{1}<0$ at any finite maturity dates for both models (13) and (16) (see appendices C and D). As regards model (13), Dai and Singleton (2000, table II) estimated that $\lambda_{v}<0$ and $\sigma_{r v}>0$ and in appendix D, I showed that $u_{2}>0$ at short maturity dates. It then follows that $\xi>0$ for short maturity dates. As regards model (16), Dai and Singleton (2000, table III) estimated that $\lambda_{v}>0$ and $\sigma_{r v}<0$ and in appendix D, I showed that $u_{2}<0$ at any finite maturity dates. It then follows that $\xi>0$ for any finite maturity date. $\|$ 


\section{Appendix G: construction of a counter-example to the strong term- structure augmenting volatility property}

Consider the well-known one-factor Gaussian model of Merton (1973) model in which $b(x)=\beta_{0}$ and $2 a(x)=\eta^{2}$. The solution for the bond price of this model is:

$u^{m}\left(x, t, T ; \frac{\eta}{2}(T-t)^{2}, \frac{\eta^{2}}{6}(T-t)^{3}\right)=\exp \left\{-x(T-t)-\left(\beta_{0}+\lambda \eta\right) \frac{(T-t)^{2}}{2}+\eta^{2} \frac{(T-t)^{3}}{6}\right\}$.

It implies that:

$$
\left\{\begin{array}{l}
\frac{\partial u^{m}}{\partial \eta^{2}}<0 \text { for } T<t+\frac{3}{4} \frac{\lambda}{\sqrt{\eta^{2}}} \\
\frac{\partial u^{m}}{\partial \eta^{2}}>0 \text { for } T>t+\frac{3}{4} \frac{\lambda}{\sqrt{\eta^{2}}}
\end{array}\right.
$$

This suggests to try to construct the counter-example I am looking for by appropriately extending the Merton's setting.

Thusly motivated, I consider the following model:

$$
\left\{\begin{array}{l}
d r(\tau)=\left(\beta_{0}+\lambda \sqrt{y(\tau)}\right) d \tau+\sqrt{y(\tau)} \cdot d W(\tau), \tau \in(t, T] \\
d y(\tau)=\varphi(y(\tau)) d t+\sqrt{\psi(y(\tau))} \cdot d W^{y}(\tau), \tau \in(t, T] \\
r(t)=x, y(t)=s
\end{array}\right.
$$

where $\beta_{0}$ and $\lambda$ are positive constants and $\varphi, \psi$ satisfy the usual regularity conditions.

I imitate the approach followed by Hull and White (1987) to extend the Black and Scholes (1973) formula to the stochastic volatility case. Specifically, I wish to express the bond price as the expectation of formula (G1) in which the constant volatility is replaced with a measure of a corresponding average, random volatility.

Consider the flow of the short-term rate process in (G3):

$$
\vartheta(\tau)=x+\beta_{0}(\tau-t)+\lambda \int_{t}^{\tau} \sqrt{\phi(u)} d u+\int_{t}^{\tau} \sqrt{\phi(u)} d W(u),
$$

and for each $u \in[t, T]$, define

$$
\left\{\begin{array}{l}
v^{1}(t, u) \equiv \int_{t}^{u} \sqrt{\phi(\tau)} d \tau \\
v^{2}(t, u) \equiv \int_{t}^{u} \phi(\tau) d \tau
\end{array}\right.
$$

Conditionally on the volatility path $(\phi(\tau))_{\tau \in[t, T]}, \vartheta(\tau)$ is normally distributed. Furthermore, $\int_{t}^{T} \vartheta(\tau) d \tau$ is also normally distributed conditionally on the volatility path $\phi$, since $\int \vartheta$ is the limit of Riemann sums that are normal:

$$
\int_{t}^{T} \vartheta(\tau) d \tau /(\phi(\tau))_{\tau \in[t, T]} \sim N\left(x(T-t)+\beta_{0} \frac{(T-t)^{2}}{2}+\lambda \int_{t}^{T} v^{1}(t, \tau) d \tau ; V^{\vartheta}(t, T)\right),
$$


where

$$
\begin{aligned}
V^{\vartheta}(t, T) & \equiv \operatorname{var}\left(\int_{t}^{T} \vartheta(\tau) d \tau /(\phi(\tau))_{\tau \in[t, T]}\right) \\
& =\int_{t}^{T} \int_{t}^{T} \operatorname{cov}\left\{\vartheta\left(\tau^{1}\right), \vartheta\left(\tau^{2}\right) /(\phi(\tau))_{\tau \in[t, T]}\right\} d \tau^{1} d \tau^{2} \\
& =\int_{t}^{T} \int_{t}^{T} v^{2}\left(t, \tau^{1} \wedge \tau^{2}\right) d \tau^{1} d \tau^{2} \\
& =2 \int_{t}^{T} \int_{t}^{\tau} v^{2}(t, \tau) d u d \tau
\end{aligned}
$$

The Feynman-Kac representation (4) can then be written here as:

$$
\begin{aligned}
u(x, s, t, T) & =\mathbb{E}\left\{\exp \left(-\int_{t}^{T} \vartheta(\tau) d \tau\right)\right\} \\
& =\mathbb{E}\left\{\mathbb{E}\left\{\exp \left(-\int_{t}^{T} \vartheta(\tau) d \tau\right) /(\phi(\tau))_{\tau \in[t, T]}\right\}\right\} \\
& =\mathbb{E}\left\{u^{m}\left(x, t, T ; \int_{t}^{T} v^{1}(t, \tau) d \tau, \int_{t}^{T} \int_{t}^{\tau} v^{2}(t, \tau) d u d \tau\right)\right\},
\end{aligned}
$$

where $u^{m}$ is, consistently with the notation introduced in formula (G1),

$$
\begin{aligned}
& u^{m}\left(x, t, T ; \int_{t}^{T} v^{1}(t, \tau) d \tau, \int_{t}^{T} \int_{t}^{\tau} v^{2}(t, \tau) d u d \tau\right) \\
= & \exp \left(-x(T-t)-\beta_{0} \frac{(T-t)^{2}}{2}-\lambda \int_{t}^{T} v^{1}(t, \tau) d \tau+\int_{t}^{T} \int_{t}^{\tau} v^{2}(t, \tau) d u d \tau\right) .
\end{aligned}
$$

I assume, by mimicking the constant volatility Merton's formula (G1), that

$$
\lim _{T \rightarrow \infty} u^{m}\left(x, t, T ; \int_{t}^{T} v_{1}(t, \tau) d \tau, \int_{t}^{T} \int_{t}^{\tau} v^{2}(t, \tau) d u d \tau\right)=\infty .
$$

We have:

Proposition G1. There exists a finite $T^{*}$ depending on $(x, s)$ such that bond prices are strictly decreasing in volatility for all maturity dates less than $T^{*}$. Suppose further that $\inf _{\tau} y(\tau)>\underline{y}$ and $\sup _{\tau} y(\tau)<\bar{y}$ a.s., where $\underline{y}$ and $\bar{y}$ are positive constants; then there exists a finite $T^{* *}$ and finite values of the short-term rate and its instantaneous volatility such that bond prices are strictly less than one and increasing in volatility at maturity date $T^{* *}$. In all cases, bond prices are decreasing and convex in the short-term rate. 
Proof. Eq. (G4) shows that $\partial \vartheta(\tau) / \partial x=1$ and $\partial^{2} \vartheta(\tau) / \partial x^{2}=0$ for all $\tau$. Substituting this into eqs. (5)-(6) leaves, for $\tau \in[t, T]$ and $\omega \in \Omega$,

$$
\left\{\begin{array}{l}
u_{1}(\vartheta(\tau), \phi(\tau), \tau, T)=-(T-\tau) \cdot u(\vartheta(\tau), \phi(\tau), \tau, T) \\
u_{11}(\vartheta(\tau), \phi(\tau), \tau, T)=(T-\tau)^{2} \cdot u(\vartheta(\tau), \phi(\tau), \tau, T)
\end{array}\right.
$$

which validates the claims of the proposition concerning the first two partial derivatives of the bond price with respect to the short-term rate.

By combining relations (G7) with lemma $\mathrm{A} 3, w^{1}$ is:

$$
w^{1}(x, s, t, T)=\mathbb{E}\left\{\int_{t}^{T} \kappa^{1}(t, \tau) \cdot u(\vartheta(\tau), \phi(\tau), \tau, T)(T-\tau)\left(T-\tau-\frac{\lambda}{2} \phi(\tau)^{-1 / 2}\right) d \tau\right\},
$$

where

$$
\kappa^{1}(t, \tau)=\exp \left\{-\int_{t}^{\tau}\left(\vartheta_{(1)}(u)-\varphi_{2}\left(\vartheta_{(1)}(u), \phi_{(1)}(u)\right)\right) d u\right\}
$$

and $\left(\vartheta_{(1)}, \phi_{(1)}\right)$ are solutions of

$$
\left\{\begin{array}{l}
d \vartheta_{(1)}=\left(\beta_{0}+\lambda \sqrt{\phi_{(1)}}\right) d \tau+\sqrt{\phi_{(1)}} d W \\
d \phi_{(1)}=\left(\varphi+\psi_{2}\right) d \tau+\sqrt{2 \psi} d W^{y}
\end{array}\right.
$$

For ease of exposition, I shall write $(\vartheta, \phi)$ instead of $\left(\vartheta_{(1)}, \phi_{(1)}\right)$ in the sequel, no confusion should arise.

Introduce the deterministic functions

$$
\left\{\begin{array}{l}
\mathcal{G}_{1}(t, \tau, T) \equiv \mathbb{E}\left\{\left(\kappa^{1}(t, \tau) \cdot u(\vartheta(\tau), \phi(\tau), \tau, T)\right)\right\} \\
\mathcal{G}_{2}(t, \tau, T) \equiv \frac{\lambda}{2} \cdot \mathbb{E}\left\{\kappa^{1}(t, \tau) \cdot u(\vartheta(\tau), \phi(\tau), \tau, T) \phi(\tau)^{-1 / 2}\right\}
\end{array}\right.
$$

and rewrite relation (G8) as:

$$
w^{1}(x, s, t, T)=\int_{t}^{T} \mathcal{G}_{1}(t, \tau, T) \cdot(T-\tau) \cdot\left(T-\tau-\frac{\mathcal{G}_{2}}{\mathcal{G}_{1}}(t, \tau, T)\right) d \tau .
$$

The existence of a maturity date $T^{*}$ can now be shown by adapting the general framework of appendix $\mathrm{C}$ to the model considered here. Indeed, by the intermediate value theorem there exists a function $\widehat{\tau}(t, T) \in[t, T]$ such that

$w^{1}(x, s, t, T)=(T-t) \cdot\left\{\mathcal{G}_{1}(t, \widehat{\tau}(t, T), T) \cdot(T-\widehat{\tau}(t, T))\right\} \cdot\left\{T-\widehat{\tau}(t, T)-\frac{\mathcal{G}_{2}}{\mathcal{G}_{1}}(t, \widehat{\tau}(t, T), T)\right\}$. 
Furthermore, $\widehat{\tau}(t, \cdot)$ is continuous, $\lim _{T \downarrow t} \widehat{\tau}(t, T)=t$ and $\lim _{T \downarrow t}(T-\widehat{\tau}(t, T))=0$ (which confirms once again lemma A5). Furthermore,

$$
\begin{aligned}
& \lim _{T \downarrow t} \frac{\mathcal{G}_{2}}{\mathcal{G}_{1}}(t, \widehat{\tau}(t, T), T) \\
= & \frac{\lambda}{2} \lim _{T \downarrow t} \frac{\mathbb{E}\left\{\kappa^{1}(t, \widehat{\tau}(t, T)) \cdot u(\vartheta(\widehat{\tau}(t, T)), \phi(\widehat{\tau}(t, T)), \widehat{\tau}(t, T), T) \cdot \phi(\widehat{\tau}(t, T))^{-1 / 2}\right\}}{\mathbb{E}\left\{\kappa^{1}(t, \widehat{\tau}(t, T)) \cdot u(\vartheta(\widehat{\tau}(t, T)), \phi(\widehat{\tau}(t, T)), \widehat{\tau}(t, T), T)\right\}} \\
= & \frac{\lambda}{2 \sqrt{s}} \\
> & 0 .
\end{aligned}
$$

This, and the continuity of $\widehat{\tau}(t, \cdot)$, imply that there exists a $T^{*}$ such that $\forall T \in\left[t, T^{*}\right]$,

$$
T^{*}-\widehat{\tau}\left(t, T^{*}\right)<\frac{\mathcal{G}_{2}}{\mathcal{G}_{1}}\left(t, \widehat{\tau}\left(t, T^{*}\right), T^{*}\right) .
$$

Comparing this with relation (G9) shows that $\forall T \in\left[t, T^{*}\right], w^{1}<0$.

To show that there exists a $T^{* *}: w^{1}>0$, I introduce the more cumbersome notation $\mathcal{G}_{1}(t, \tau, T, x, s) \equiv \mathcal{G}_{1}(t, \tau, T)$, and define for each finite $T$,

$$
\mathbb{G}_{T} \equiv\left\{(x, s) \in \mathbb{R}_{+} \times \mathbb{R}: \mathcal{G}_{1}(t, \tau, T, x, s) \in(0,1), \text { all } \tau \in[t, T]\right\},
$$

and

$$
\mathbb{G}_{\infty} \equiv\left\{(x, s) \in \mathbb{R}_{+} \times \mathbb{R}: \mathcal{G}_{1}(t, \tau, \infty, x, s)>0, \text { all } \tau \geq t\right\},
$$

The general pricing formula (G5) and condition (G6) clearly show that $\mathbb{G}_{T}$ and $\mathbb{G}_{\infty}$ are not empty. Then, for each $\left(x_{T}, s_{T}\right) \in \mathbb{G}_{T}$, I introduce the following function:

$$
\ell(T) \equiv t+\frac{3 \delta}{2} \frac{1}{\min _{\tau \in[t, T]} \mathcal{G}_{1}\left(t, \tau, T ; x_{T}, s_{T}\right)},
$$

where $\delta \equiv \frac{\lambda}{2} \Theta(\underline{y})^{-1 / 2}$ and $\Theta(\underline{y})$ is a deterministic function of $\underline{y}$ defined as the left supporting point of the auxiliary process $\phi_{(1)}$, id est: $\Theta(\underline{y})<\inf _{\tau} \phi_{(1)}(\omega ; \tau)$ all $\omega \in \Omega$ and $\tau \in[t, T]$. Clearly, $\Theta(\underline{y})$ is bounded because $\inf _{\tau} \phi(\tau)>\underline{y}$ a.s.

Function $\ell$ is continuous and by construction, for each sequence $\left(x_{n}, s_{n}\right)$ converging to elements of $\mathbb{G}_{\infty}$,

$$
\lim _{n \rightarrow \infty} \min _{\tau \in[t, n]} \mathcal{G}_{1}\left(t, \tau, n ; x_{n}, s_{n}\right) \in(0,1),
$$

and hence $\ell$ has a fixed point $T^{* *}=\ell\left(T^{* *}\right)$. I claim that for each $\left(x_{T}, s_{T}\right) \in \mathbb{G}_{T}$, such a fixed point $T^{* *}$ can be taken as a candidate expiration date of the kind that was claimed to exist in the proposition. Notice indeed that

$$
w^{1}(x, s, t, T) \geq \int_{t}^{T} \mathcal{G}_{1}(t, \tau, T)(T-\tau)(T-\tau-\delta) d \tau
$$


and the condition that the right hand side of the preceding inequality is strictly positive is:

$$
\frac{\int_{t}^{T} \mathcal{G}_{1}(t, \tau, T)(T-\tau)^{2} d \tau}{\int_{t}^{T} \mathcal{G}_{1}(t, \tau, T)(T-\tau) d \tau}>\delta
$$

Now

$$
\frac{\int_{t}^{T} \mathcal{G}_{1}(t, \tau, T)(T-\tau)^{2} d \tau}{\int_{t}^{T} \mathcal{G}_{1}(t, \tau, T)(T-\tau) d \tau}>\frac{2}{3} \frac{\min _{\tau \in[t, T]} \mathcal{G}_{1}(t, \tau, T)}{\max _{\tau \in[t, T]} \mathcal{G}_{1}(t, \tau, T)}(T-t) .
$$

By evaluating the preceding inequality at $T^{* *}$ using any $\left(x_{T^{* *}}, s_{T^{* *}}\right) \in \mathbb{G}_{T^{* *}}$, and noting that by construction $\max _{\tau \in\left[t, T^{* *}\right]} \mathcal{G}_{1}\left(t, \tau, T^{* *} ; x_{T^{* *}}, s_{T^{* *}}\right)<1$, one obtains exactly inequality (G11). The claim that the bond price is less than one at $T^{* *}$ follow from the fact that for each $\left(x_{T^{* *}}, s_{T^{* *}}\right) \in \mathbb{G}_{T^{* *}}, \mathcal{G}_{1}\left(t, t, T^{* *}, x_{T^{* *}}, x_{T^{* *}}\right)=u\left(x_{T^{* *}}, s_{T^{* *}}, t, T^{* *}\right)<1 . \|$

Finally, please note the existence of a formal analogy between the critical maturity date in (G2) and the fixed point of function (G10) that I rewrite as:

$$
\ell(T) \equiv t+\frac{3}{4} \frac{\lambda}{\sqrt{\Theta(\underline{y})}} \frac{1}{\min _{\tau \in[t, T]} \mathbb{E}\left\{\kappa^{1}(t, \tau) \cdot u(\vartheta(\tau), \phi(\tau), \tau, T)\right\}} .
$$




\section{References}

Aït-Sahalia, Y., 1996a, "Testing Continuous-Time Models of the Spot Interest Rate," Review of Financial Studies, 9, 385-426.

Aït-Sahalia, Y., 1996b, "Nonparametric Pricing of Interest Rate Derivative Securities," Econometrica, 64, 527-560.

Ahn, C.-M. and H. E. Thompson, 1988, "Jump-Diffusion Processes and the Term Structure of Interest Rates," Journal of Finance, 43, 155-174.

Ahn, D.-H. and B. Gao, 1999, "A Parametric Nonlinear Model of Term Structure Dynamics," Review of Financial Studies, 12, 721-762.

Andersen, T. G. and J. Lund, 1997a, "Estimating Continuous-Time Stochastic Volatility Models of the Short-Term Interest Rate," Journal of Econometrics, 77, 343-377.

Andersen, T. G. and J. Lund, 1997b, "Stochastic Volatility and Mean Drift in the Short Rate Diffusion: Sources of Steepness, Level, and Curvature in the Yield Curve," unpublished manuscript, Aarhus University, Denmark.

Bajeux-Besnainou, I. and Jean-Charles Rochet, 1996, "Dynamic Spanning: Are Options an Appropriate Instrument ?," Mathematical Finance, 6, 1-16.

Bakshi, G., C. Cao and Z. Chen, 2000, "Do Call Prices and the Underlying Stock Always Move in the Same Direction ?," Review of Financial Studies, 13, 549-584.

Balduzzi, P., S. R. Das, S. Foresi, and R. K. Sundaram, 1996, "A Simple Approach to Three Factor Affine Term Structure Models," Journal of Fixed Income, 6, 43-53.

Bergman, Y. Z., B. D. Grundy, and Z. Wiener, 1996, "General Properties of Option Prices," Journal of Finance, 51, 1573-1610.

Black, F. and M. Scholes, 1973, "The Pricing of Options and Corporate Liabilities," Journal of Political Economy, 81, 637-659.

Boudoukh, J., M. Richardson, R. Stanton, and R. F. Whitelaw, 1998, "The Stochastic Behavior of Interest Rates: Implications from a Multifactor, Nonlinear Continuous-Time Model," unpublished manuscript, Haas School of Business, UC Berkeley.

Campbell, J. Y., A. W. Lo, and A. C. MacKinlay, 1997, The Econometrics of Financial Markets, Princeton University Press, Princeton, NJ.

Chan, K. C., G. A. Karolyi, F. A. Longstaff, and A. B. Sanders, 1992, "An Empirical Comparison of Alternative Models of Short-Term Interest Rate," Journal of Finance, 47, 1209-1228.

Chapman, D. A., J. B. Long, and N. D. Pearson, 1999, "Using Proxies for the Short Rate: When Are Three Months like an Instant?," Review of Financial Studies, 12, 763-806. 
Chernov, M. and E. Ghysels, 2000, "A Study towards a Unified Approach to the Joint Estimation of Objective and Risk-Neutral Measures for the Purpose of Options Valuation," Journal of Financial Economics, 56, 407-458.

Chen, L., 1996, Interest Rate Dynamics, Derivative Pricing, and Risk Management, Springer: Lecture Notes in Economics and Mathematical Systems n. 435, Berlin.

Conley, T. G., L. P. Hansen, E. G. J. Luttmer, and J. A. Scheinkman, 1997, "Short-Term Interest Rates as Subordinated Diffusions," Review of Financial Studies, 10, 525-577.

Cox, J. C., and S. A. Ross, 1976, "A Survey of Some New Results in Financial Option Pricing Theory," Journal of Finance, 31, 383-402.

Cox, J. C., J. E. Ingersoll, and S. A. Ross, 1985, "A Theory of the Term Structure of Interest Rates," Econometrica, 53, 385-407.

Dai, Q. and K. J. Singleton, 2000, "Specification Analysis of Affine Term Structure Models," Journal of Finance, 55, 1943-1978.

Das, S. R., 2000, "The Surprise Element: Jumps in Interest Rates," mimeo, Santa Clara University.

Duffie, D., 1996, Dynamic Asset Pricing Theory, 2nd ed., Princeton University Press, Princeton, NJ.

Duffie, D. and K. Singleton, 1999, "Modeling Term Structures of Defaultable Bonds," Review of Financial Studies, 12, 687-720.

Duffie, D., J. Pan and K. Singleton, 2000, "Transform Analysis and Asset Pricing for Affine Jump-Diffusions," Econometrica, 68, 1343-1376.

Dunn, K. B. and C. S. Spatt, 1999, "Call Options, Points, and Dominance Restrictions on Debt Contracts," Journal of Finance, 54, 2317-2337.

Dybvig, P., J. E. Ingersoll, Jr., and S. A. Ross, 1996, "Long Forward and Zero-Coupon Rates Can Never Fall, Journal of Business, 69, 1-25.

El Karoui, N., M. Jeanblanc-Picqué and S. E. Shreve, 1998, "Robustness of the Black and Scholes Formula," Mathematical Finance, 8, 93-126.

Fong, H. G. and O. A. Vasicek, 1991, "Fixed Income Volatility Management," The Journal of Portfolio Optimization, Summer issue, 41-46.

Fouque, J.-P., G. Papanicolaou and K. R. Sircar, 2000, Derivatives in Financial Markets with Stochastic Volatility, Cambridge University Press, Cambridge.

Friedman, A., 1975, Stochastic Differential Equations and Applications, Academic Press, New York, Vol. 1. 
Gallant, A. R. and G. Tauchen, 1998, "Reprojecting Partially Observed Systems with Applications to Interest Rate Diffusions," Journal of American Statistical Association, 93, 10-24.

Gard, T. C., 1988, Introduction to Stochastic Differential Equations, M. Dekker, New York.

Ghysels, E., A. Harvey, and E. Renault, 1996, "Stochastic Volatility," in C. R. Rao (ed.), Handbook of Statistics, Vol.14: Statistical Methods in Finance, 119-191, Elsevier, North-Holland, Amsterdam.

Heath, D., R. Jarrow, and A. Morton, 1992, "Bond Pricing and the Term Structure of Interest Rates: a New Methodology for Contingent Claims Valuation," Econometrica, $60,77-105$.

Hull, J. C. and A. D. White, 1987, "The Pricing of Options with Stochastic Volatilities," Journal of Finance, 42, 281-300.

Jacod, J. and A. N. Shiryaev, 1987, Limit Theorems for Stochastic Processes, Springer Verlag, Berlin.

Jagannathan, R., 1984, "Call Options and the Risk of Underlying Securities," Journal of Financial Economics, 13, 425-434.

Karatzas, I. and S. E. Shreve, 1991, Brownian Motion and Stochastic Calculus, Springer Verlag, Berlin.

Karlin, S. and H. M. Taylor, 1981, A Second Course in Stochastic Processes, Academic Press, San Diego.

Krylov, N. V., 1980, Controlled Diffusion Processes, Springer Verlag, Berlin.

Kunita, H., 1990, Stochastic Flows and Stochastic Differential Equations, Cambridge University Press, Cambridge.

Lando, D., 1998, "On Cox Processes and Credit Risky Securities," Review of Derivatives Research, 2, 99-120.

Lewis, A. L., 2000, Option Valuation Under Stochastic Volatility, Finance Press, Newport Beach, CA.

Litterman, R., J. Scheinkman, and L. Weiss, 1991, "Volatility and the Yield Curve," Journal of Fixed Income, June, 49-53.

Longstaff, F. A., 1989, "A Nonlinear General Equilibrium Model of the Term-Structure of Interest Rates," Journal of Financial Economics, 23, 195-224.

Longstaff, F. A., 1992, "Multiple Equilibria and Term Structure Models," Journal of Financial Economics, 32, 333-344. 
Longstaff, F. A. and E. S. Schwartz, 1992, "Interest Rate Volatility and the Term Structure: a Two-Factor General Equilibrium Model," Journal of Finance, 47, 1259-1282.

Longstaff, F. A. and E. S. Schwartz, 1993, "Implementation of the Longstaff-Schwartz Interest Rate Model," Journal of Fixed Income, September, 7-14.

Mele, A., and F. Fornari, 2000, Stochastic Volatility in Financial Markets: Crossing the Bridge to Continuous Time, Kluwer Academic Publishers, Boston.

Mele, A., and F. Fornari, 2001, "Recovering the Probability Density Function of Asset Prices Using ARCH Models as Diffusion Approximations," Journal of Empirical Finance, 8, 83-110.

Merton, R. C., 1973, "Theory of Rational Option Pricing," Bell Journal of Economics and Management Science, 4, 141-183.

Romano, M. and N. Touzi, 1997, "Contingent Claims and Market Completeness in a Stochastic Volatility Model," Mathematical Finance, 7, 399-412.

Rothschild, M. and J.E. Stiglitz, 1970, "Increasing Risk: I. A Definition," Journal of Economic Theory, 2, 225-243.

Stanton, R., 1997, "A Nonparametric Model of Term Structure Dynamics and the Market Price of Interest Rate Risk," Journal of Finance, 52, 1973-2002.

Vasicek, O., 1977, An Equilibrium Characterization of the Term Structure, Journal of Financial Economics, 5, 177-188. 


\section{Q. Queen Mary \\ University of London}

This working paper has been produced by the Department of Economics at Queen Mary, University of London

Copyright @ 2002 Antonio Mele All rights reserved.

\section{Department of Economics}

Queen Mary, University of London

Mile End Road

London E1 4NS

Tel: +44 (0)20 78825096 or Fax: +44 (0)20 89833580

Email: j.conner@qmul.ac.uk

Website: www.econ.qmul.ac.uk/papers/wp.htm 